\title{
Doing it for the 'Gram: How Instagram affects values, and what it means for mental and ecological wellbeing
}

\author{
Melanie Vautier
}

A thesis submitted to Victoria University of Wellington, Te Herenga Waka, in partial fulfilment of the requirements for the degree of Master of Environmental Studies

School of Geography, Environment, and Earth Sciences 


\begin{abstract}
What would it mean to cultivate a culture of thriving people on a thriving planet? Research has shown that intrinsic values are a significant predictor of both environmentally responsible behaviour and mental wellbeing. However, in modern Western culture, extrinsic, self-promotional values continue to grow in prominence. In order to examine how a specific social context may be exacerbating extrinsic values, counter to mental and ecological wellbeing, this study looks at the social media platform Instagram. Through in-depth interviews of young adults in Aotearoa New Zealand, this study examines their experiences of Instagram in order to ascertain if and how it may promote extrinsic values. Results were analysed through Self Determination Theory, which states that environments which thwart basic human needs of relatedness, competence and autonomy will incentivise extrinsic values. While in some cases, Instagram increased access to relatedness, participants also experienced thwarted relatedness when interactions became motivated predominantly by posting them, and thus self-promoting, on the platform. Participants also consistently reported feeling incompetent when comparing themselves to others' images, which, particularly when adjacent to advertising, as in the case of Instagram, is likely to lead to materialistic tendencies. Participants were also aware of, and in some cases responded to, Instagram signals as to what type of content would gain more 'likes,' adjusting their behaviour in order to succeed in a 'marketplace' infrastructure, thereby limiting autonomy. In doing so, intrinsically motivated experiences were overtaken by an extrinsic desire to portray them to others. These examples indicate Instagram's infrastructural incentives toward extrinsic values, which counter societal goals of mental and ecological wellbeing. Pervasive messaging within social contexts such as Instagram needs to shift away from promoting extrinsic values in order to respond adequately to the current ecological challenges and create conditions conducive to mental wellbeing.
\end{abstract}




\section{Acknowledgements}

Thank you to my supervisor Wokje Abrahamse for all your feedback and for bearing with me through countless evolutions of this thesis.

To all the participants, thank you enormously for freely giving up your time and sharing your experiences so openly. This thesis would not exist without you.

To the incredible co-inhabitants of CO102, thank you for the warm fuzzies, the support, the happy hours, and the ukulele raps.

To my amazing flatmates: Thank you for your unfailing support, for the shared meals, chats, laughs, dances, and for making Welly home for me. To my friends: Thank you for always giving me hope in humanity all throughout the often-alarming subject material of this degree.

To my wonderful family who made all of this possible and supported me to pursue my passions, thank you. 


\section{Contents}

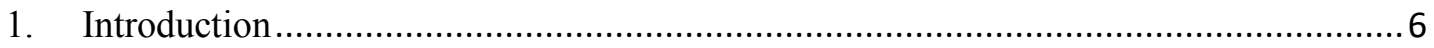

2. Theoretical frameworks: Exploring values .............................................................. 11

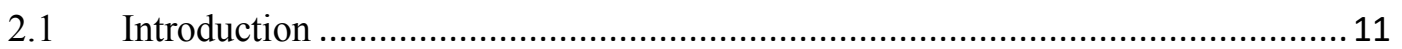

2.2 Links between values and environmentally responsible behaviour ......................11

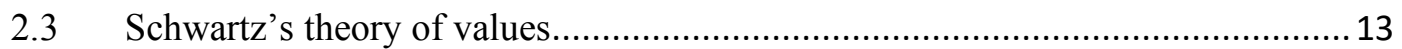

2.4. Introducing self-determination theory ................................................................. 16

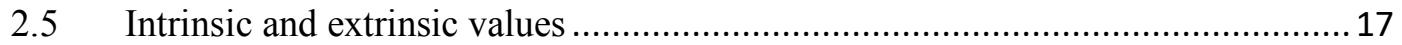

2.6 Congruence between Schwartz and SDT ..................................................... 21

2.7 Linking mental and ecological wellbeing through values ..................................22

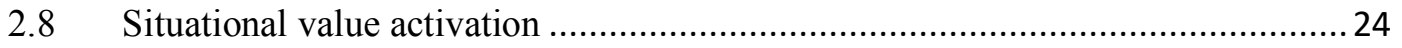

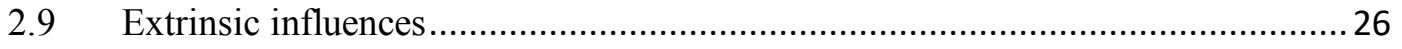

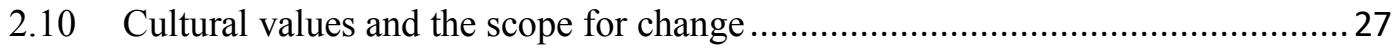

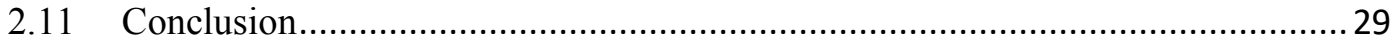

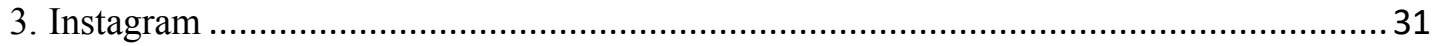

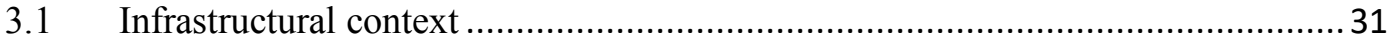

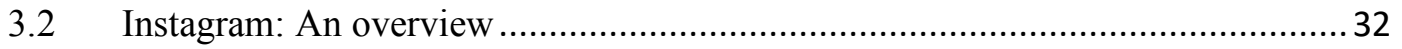

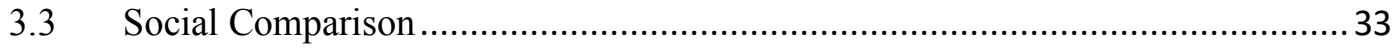

3.4 Arguments of self-commodification through Instagram ..................................... 34

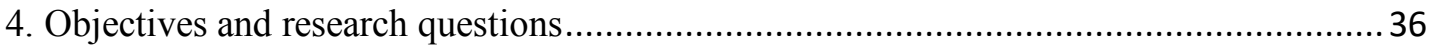

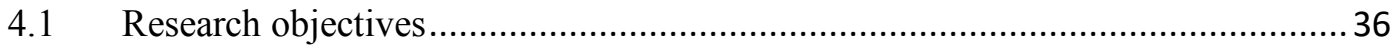

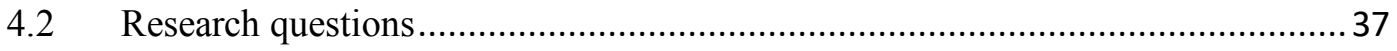

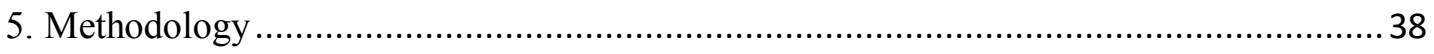

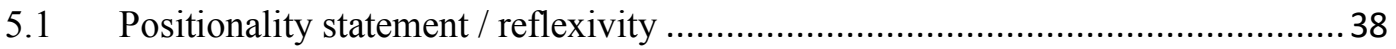

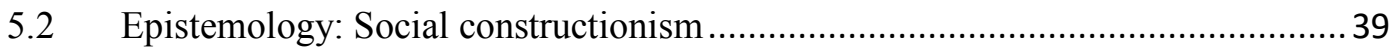

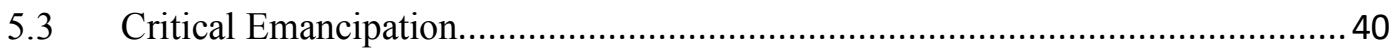

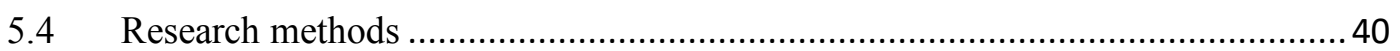

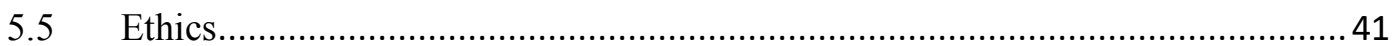

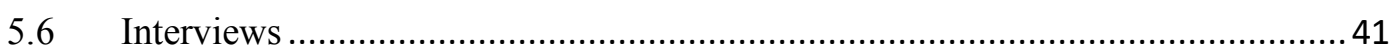

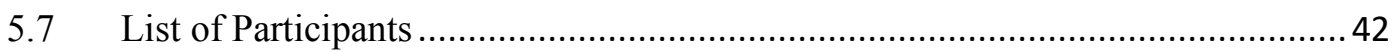

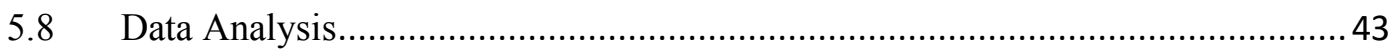

6. The "social" in social media: Instagram and Relatedness ................................................ 45

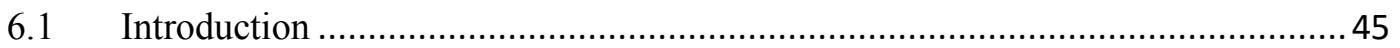

6.2 Acceptance and identity in online communities ................................................46 


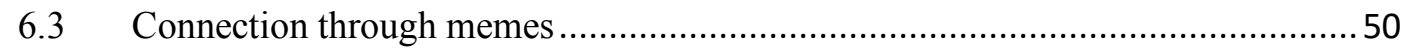

6.4 Unsatisfying online connection and the need for external gratification................53

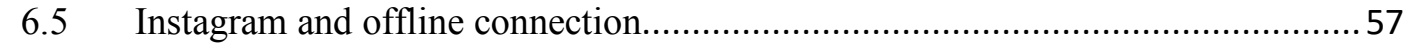

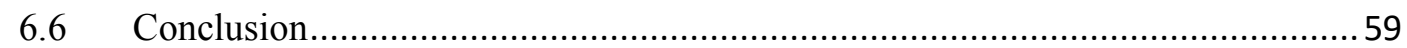

7. Social comparison, thwarted competence and materialism ..........................................61

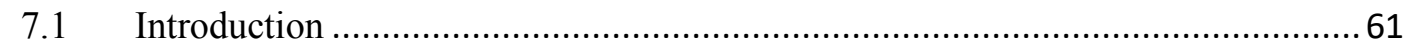

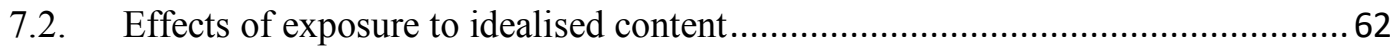

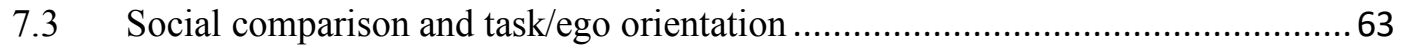

7.4 Social comparison with friends vs celebrities...................................................6 66

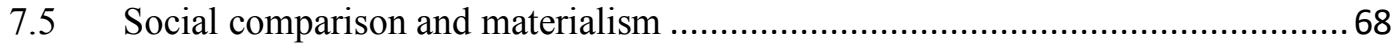

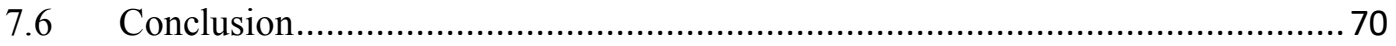

8. Autonomy: Incentives toward self-promotion ……................................................. 72

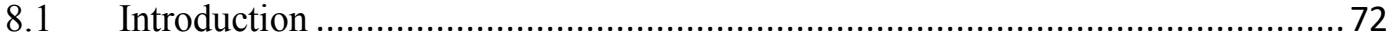

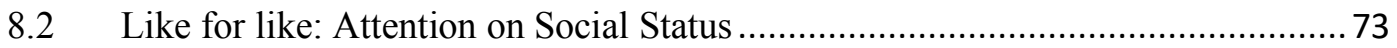

8.3 "What they want to see:" Following the demands of the 'market'......................... 74

8.4 Infrastructural incentives toward self-promotion and self-objectification ............. 77

8.5 "Do it for the Gram:" from intrinsic experiences to extrinsic representations........81 81

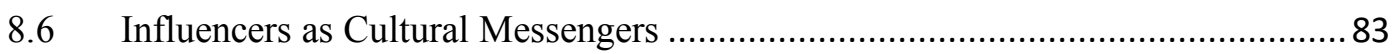

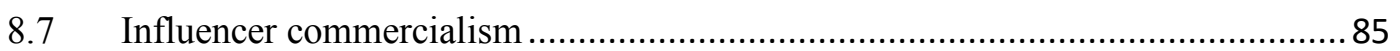

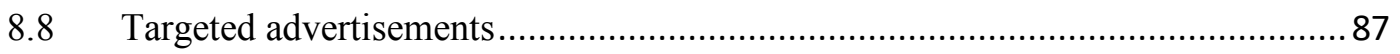

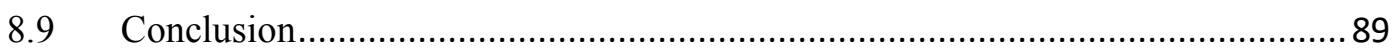

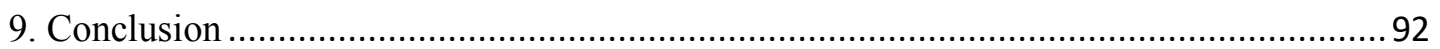

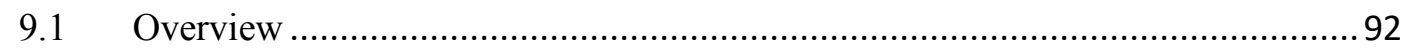

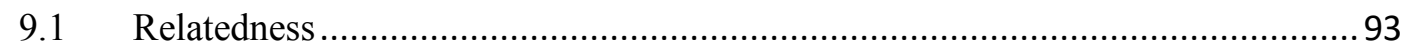

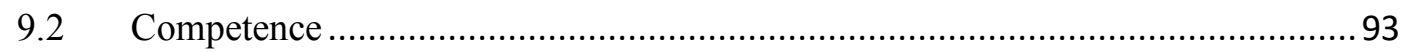

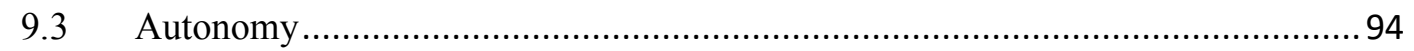

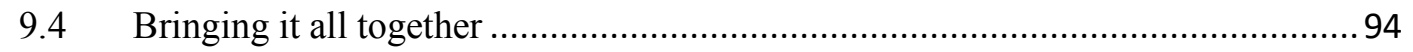

9.5 Implications for mental and ecological well-being ........................................... 95

9.6 Limitations and Future Directions ............................................................... 96

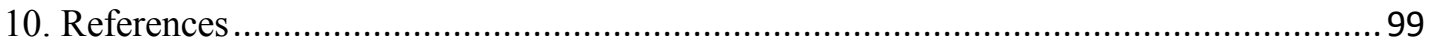

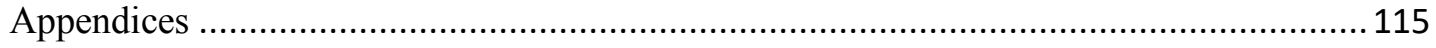

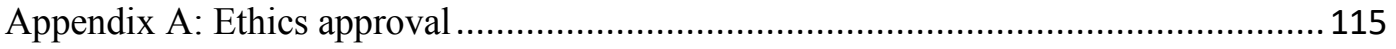

Appendix B: Participant information and consent form .............................................. 116

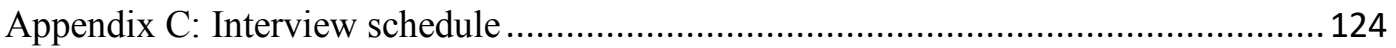




\title{
1. Introduction
}

\author{
"It became increasingly obvious to me that the entire debate needs to \\ shift; and it cannot do so unless we figure out what it is we are reaching \\ towards - the underpinning values that we want to live by and the vision \\ of where we are going that makes our actions make sense." \\ -Dr. Niki Harré, Professor of Psychology (2018, p. 14)
}

In a 2018 press release, the Intergovernmental Panel on Climate Change stated that avoiding catastrophic climate change, "would require rapid, far-reaching and unprecedented changes in all aspects of society" (IPCC, 2018). However, two years on, greenhouse gas emissions continue to rise, with global negotiations on what to about it at a stalemate (Streck et al., 2020). The disastrous consequences longpredicted by climate scientists are already coming to fruition, with unprecedented temperatures and increased extreme weather events (Boer et al., 2020; Diffenbaugh, 2020; Vogel et al., 2015). Scientists are growing increasingly urgent in their warnings, with 11,000 scientists from around the world signing a declaration in 2019 stating "clearly and unequivocally that planet Earth is facing a climate emergency" (Ripple et al., 2020, p. 8). Still, the societal change advocated for by the IPCC remains absent from mainstream discourse.

Moreover, climate change is just one of multiple current ecological crises. Humanity is currently in the midst of the sixth major extinction event in history, termed a "biological annihilation" (Ceballos et al., 2017, p. 1). Further, freshwater consumption, ocean acidification, the atmospheric nitrogen and phosphorus cycles, and ozone depletion are all surpassing safe ecological limits (Rockström et al., 2009). All these issues are driven by humans, in particular through overconsumption in affluent countries (Hamilton, 2004; Jackson, 2017; Raworth, 2017).

A growing body of research argues that responses to these environmental crises necessitate a shift in the human values that underpin behaviours and attitudes (Crompton, 2010; Hurst et al., 2013; Kasser, 2016; Rockström et al., 2009; Schultz 
et al., 2005). Values have been demonstrated to be an antecedent to more environmentally responsible behaviour (Davidov et al., 2008; Kasser \& Kanner, 2004; Unanue et al., 2016). Some values are closely correlated with determining a persons' level of environmental concern and carbon footprint: According to SelfDetermination Theory (SDT) (Ryan \& Deci, 2017), most values fall along a spectrum between intrinsic and extrinsic. Intrinsic values are those which are inherently interesting in and of themselves, and include values such as connection to nature, close personal relationships, or contributing to a cause or community. Studies have demonstrated that intrinsic values predict environmentally responsible behaviour even when a pro-environmental worldview or identity does not (Unanue et al., 2016). Extrinsic values are representative, motivated by and measured in praise or rewards from others, and include values such as wealth, status, or image. Extrinsic values correlate with a higher carbon footprint and lower levels of environmental concern (Hurst et al., 2013; Kasser, 2016).

Research on values demonstrates that environmentally responsible behaviour also correlates with higher levels of mental wellbeing. In contrast to pervasive framing that responding to climate change necessitates personal sacrifice, research has shown that people with relatively stronger intrinsic values report higher levels of subjective wellbeing than those with relatively stronger extrinsic values (Hurst et al., 2013; Kasser, 2016; Ryan \& Deci, 2017). Thereby, intrinsic values are indicative of being conducive to both ecological and mental wellbeing. This perspective offers a 'winwin' situation. Thereby, agents aiming to work toward goals of both mental and ecological wellbeing could do so through the support and promotion of intrinsic values.

Value orientation can shift according to contextual factors, both in an immediate sense of 'activating' values through cues within a social environment, and in a more lasting sense, through a repeated cultural emphasis signalling important aspirations in life. Citizens in modern Western culture are exposed to hundreds of messages each day in advertising, media, billboards and public discourse promoting material consumption and luxury lifestyles (Hamilton, 2005; Richins, 2017). This messaging is directly opposed to, and far more pervasive than, messaging promoting intrinsic values and environmental care. From this perspective, the lack of adequate response 
to climate change may then be unsurprising. The values continually reflected and reinforced in modern Western society conflict with what is empirically known to contribute to mental and ecological wellbeing. The societal transformation that the IPCC calls for necessitates a move away from consumer culture (Stuart et al., 2020a). This shift may begin with a discussion about core cultural values.

According to SDT, intrinsic and extrinsic goals are largely mediated by the satisfaction and frustration of three universal basic needs: Relatedness, the need to be closely connected and supported by others; competence, the need to feel capable; and autonomy, the need to be able to express oneself genuinely, without coercion (Ryan \& Deci, 2017). When these needs are frustrated, individuals are "more prone to adopt need substitutes, such as extrinsic life goals, as being personally important," to the detriment of their levels of wellbeing (Ryan \& Deci, 2017, p. 285).

SDT further states that values are influenced by the economic, political, or cultural institutions which "set psychological horizons on the very possibilities that persons within them can envision" (Ryan \& Deci, 2017, p. 562). Hence, these institutions have enormous power to influence cultural values which, in turn, shape the lives of the citizens who live within them. Values scholars Schwartz (2011) and Kasser (2016) both recommend examining various institutions to determine how they may encourage different values. In this thesis, I take this approach, focussing on the social context of Instagram.

Instagram is of particular interest for several reasons. As stated by Christiana Figueres, former Executive Secretary of the UN Framework Convention on Climate Change, global technology companies have their "hands on the levers of human behaviour" (Center for Humane Technology, 2020). The scale of Instagram means it is a globally influential social context, with over a billion users (Statista, 2020). Having risen to prominence over the past decade, research on its effects are still relatively unknown and, further, unconstrained, as regulation remains minimal ( $\mathrm{Wu}$, 2019). 43\% of Instagram users are under 25 (Statista, 2020), meaning a large demographic of users are at an impressionable age and undergoing a crucial period of identity development (Sapolsky, 2017). Infrastructurally, Instagram is an imagebased platform in which users upload images which are qualitatively recognised by 
others through the likes or number of comments an image receives. As extrinsic values, by definition, are concerned with gaining approval from others (Kasser, 2002a), this indicates Instagram may be exacerbating extrinsic orientations.

The thesis is structured as follows: Following this Introduction, in Chapter 2, I give an overview of the existing literature and theoretical frameworks underpinning this research. I discuss Schwartz's theory of values (1992), which proposes that there are values universal among all people, which interact in predictable ways. I then discuss SDT's premise that there are three fundamental human needs, which can be either supported or thwarted by socio-environmental context (Ryan \& Deci, 2017). These two theories combine within 'Goal Contents Theory' (Kasser, 2002a), which argues that values vary in terms of how they support or thwart the basic human needs of Self Determination Theory, with empirical effects on mental and ecological wellbeing.

In Chapter 3, I examine the literature on social media, with a particular focus on Instagram. I discuss the pervasiveness of social comparison on Instagram, motivations for using the platform, and literature arguing that Instagram encourages self-commodification.

Chapter 4 summarises the findings of the literature review and outlines the objectives of the research. Here, I pinpoint the 'gap' in the research, which informs my research questions.

Chapter 5 describes my methodological approach. I discuss my positionality and epistemology, followed by the research methods utilised in this thesis.

The following three chapters, $6-8$, cover my analysis of the findings and how they relate to the literature. Each chapter focusses on one of the three basic human needs according to SDT, and how participant experiences indicate ways Instagram supports or thwarts these needs.

Chapter 6 focuses on relatedness, looking at how Instagram supports or abates human connection. As one of the basic human needs and an intrinsic value, connection to others (or lack of) is a crucial predictor of value orientation. Chapter 
7 looks at how participant experiences of social comparison on Instagram led to thwarted feelings of competence, and the links between thwarted competence and increased materialism. Chapter 8 examines the infrastructural incentives which participants experienced on Instagram, and how these interact with perceptions of autonomy of self-expression on the platform.

Finally, Chapter 9 offers a conclusion, presenting a discussion summarising the main findings from the research, limitations and suggestions for future research. 


\title{
2. Theoretical frameworks: Exploring values
}

\author{
"The environmental crisis has been brought on largely by the \\ thoughts, feelings, attitudes, values and behaviours of human \\ beings"
}

(Kasser \& Kanner, 2004, p. 76)

\subsection{Introduction}

An ever-increasing body of literature recognises values for their ability to influence attitudes (Unanue et al., 2016), ideologies (Schwartz, 2011), political choices (Piurko et al., 2011), and behaviour (Crompton, 2010; Kasser \& Ryan, 1996; Ryan \& Deci, 2017). In this chapter, I outline an overview of the theories used as a basis for this thesis. First, I outline the literature demonstrating why values are a key element of responding to environmental challenges. I then discuss Schwartz's work on values, including the ten universal human values and how they interact with each other. I then discuss Self Determination Theory (SDT), developed by Ryan and Deci to describe the fundamental human needs of competence, autonomy and relatedness. Following this, I describe Kasser's work combining Schwartz's theory of values and SDT to form Goal Contents Theory, which argues that some values are more likely than others to contribute to basic human needs. I then how values link mental and ecological wellbeing, how different values can be 'activated' momentarily, and how this translates to a real-life context. Finally, I examine the relationship between cultural values and individual values, and the scope for change.

\subsection{Links between values and environmentally responsible behaviour}

Recent research has identified values as the primary determinant of an individual's environmental attitudes and personal carbon footprint (Kasser, 2016; Unanue et al., 2016). The centrality of values in many aspects of life means that they "may 
influence the direction of social change and its speed" (Davidov et al., 2008, p. 422). Therefore, it follows that values are likely to play an essential role in the transformational social change deemed necessary by the IPCC in order to avoid catastrophic climate change (2018; see Introduction)

Values are the foundation upon which attitudes and behaviours are built (Schwartz, 2011). Examining behaviours that are harmful to the environment, without considering the underlying values which predict such behaviour, cannot be comprehensive enough for the required shift to mitigate climate change (Crompton, 2010). There is evidence to suggest that even a "win" for increasing a particular proenvironmental behaviour could be, in the long term, counterproductive if it succeeds by appealing to the wrong values (Crompton, 2010; Fitzsimons, 2011). For example, a message to reduce energy use in order to gain economic savings may be successful in its endeavour. However, this behavioural change is built upon shaky ground: The message reinforces the value of money as a guiding principle, so if an individual subsequently finds a cheaper option, they will be more likely to take it, regardless of environmental outcomes. Further, appealing to economic values is likely to be detrimental to broader behaviours related to caring for the environment (for further discussion on the conflict between environmental and economic values see Sections 2.4 and 2.6). Considering the scale of ecological crises, responses must be broader than single-action-specific outcomes (Brown \& Kasser, 2005).

A longitudinal study by Unanue et al. compared three factors in order to ascertain how they each predicted environmentally responsible behaviour. These factors were environmental identification (those for whom "nature has inherent value because it is interconnected with their identities" (2016, p. 9)), pro-environmental worldviews, and extrinsic/intrinsic values. They followed 1215 adults from Britain and Chile over two years. They found that, of the three variables, the only one that was a significant predictor of environmentally responsible behaviour was intrinsic life goals. The life goal most negatively correlated with environmental behaviour was wealth, and the most positively correlated was community involvement. They suggest that "proenvironmental worldviews, as well as environmental identification, might be better understood as consequences rather than antecedents of environmentally friendly 
behaviour" (Unanue et al., 2016, p. 20). In this way, the key to increased environmental behaviours, identities and worldview may begin with intrinsic values.

Unanae et al.'s findings indicate that environmental goals may be achieved more effectively through focussing on shifting cultural values, rather than campaigning directly on environmental messaging. They recommend that public policies considering climate should focus on limiting materialistic influences while encouraging people to live a more "intrinsic and meaningful life" (2016, p. 21); a recommendation echoed by Kasser (2016) and Hurst (2013).

\subsection{Schwartz's theory of values}

Since the 1970's, values have been understood to be an interconnected, dynamic system (Kasser, 2016). Crompton and Kasser describe values as "the aspects of people's identities that reflect what they deem to be desirable, important, and worthy of striving for in their lives" (2009, p. 8). Seminal work by Schwartz (1992) proposed a universal content and structure of values consistent in all people across cultures. While each value is present in all people, the relative strength of each value varies according to each individual. These values influence goals, attitudes and behaviours. Schwartz's research on values has included hundreds of participants from dozens of countries; and been built upon by an ever-increasing number of researchers recognising the importance of values in a multitude of issues (Schwartz, 2011). The ten values recognised as universal under Schwartz's theory are as follows: 


\section{Table 1}

Schwartz's ten values and conceptual definitions (Schwartz et al., 2012, p. 664).

\begin{tabular}{|c|c|}
\hline Value & Conceptual definition ${ }^{a}$ \\
\hline Self-direction & Independent thought and action-choosing, creating, exploring \\
\hline Stimulation & Excitement, novelty, and challenge in life \\
\hline Hedonism & Pleasure and sensuous gratification for oneself. \\
\hline Achievement & Personal success through demonstrating competence according to social standards \\
\hline Power & Social status and prestige, control or dominance over people and resources \\
\hline Security & Safety, harmony, and stability of society, of relationships, and of self \\
\hline Conformity & $\begin{array}{l}\text { Restraint of actions, inclinations, and impulses likely to upset or harm others and } \\
\text { violate social expectations or norms }\end{array}$ \\
\hline Tradition & $\begin{array}{l}\text { Respect, commitment, and acceptance of the customs and ideas that traditional } \\
\text { culture or religion provides }\end{array}$ \\
\hline Benevolence & $\begin{array}{l}\text { Preservation and enhancement of the welfare of people with whom one is in } \\
\text { frequent personal contact }\end{array}$ \\
\hline Universalism & $\begin{array}{l}\text { Understanding, appreciation, tolerance and protection for the welfare of all } \\
\text { people and for nature }\end{array}$ \\
\hline
\end{tabular}

As an interconnected and dynamic system, these ten values are varyingly compatible or conflicting with each other. To communicate the interplay between different values, Schwartz arranged them in a circular, or 'circumplex,' arrangement, demonstrated in Figure 1 on the following page: 


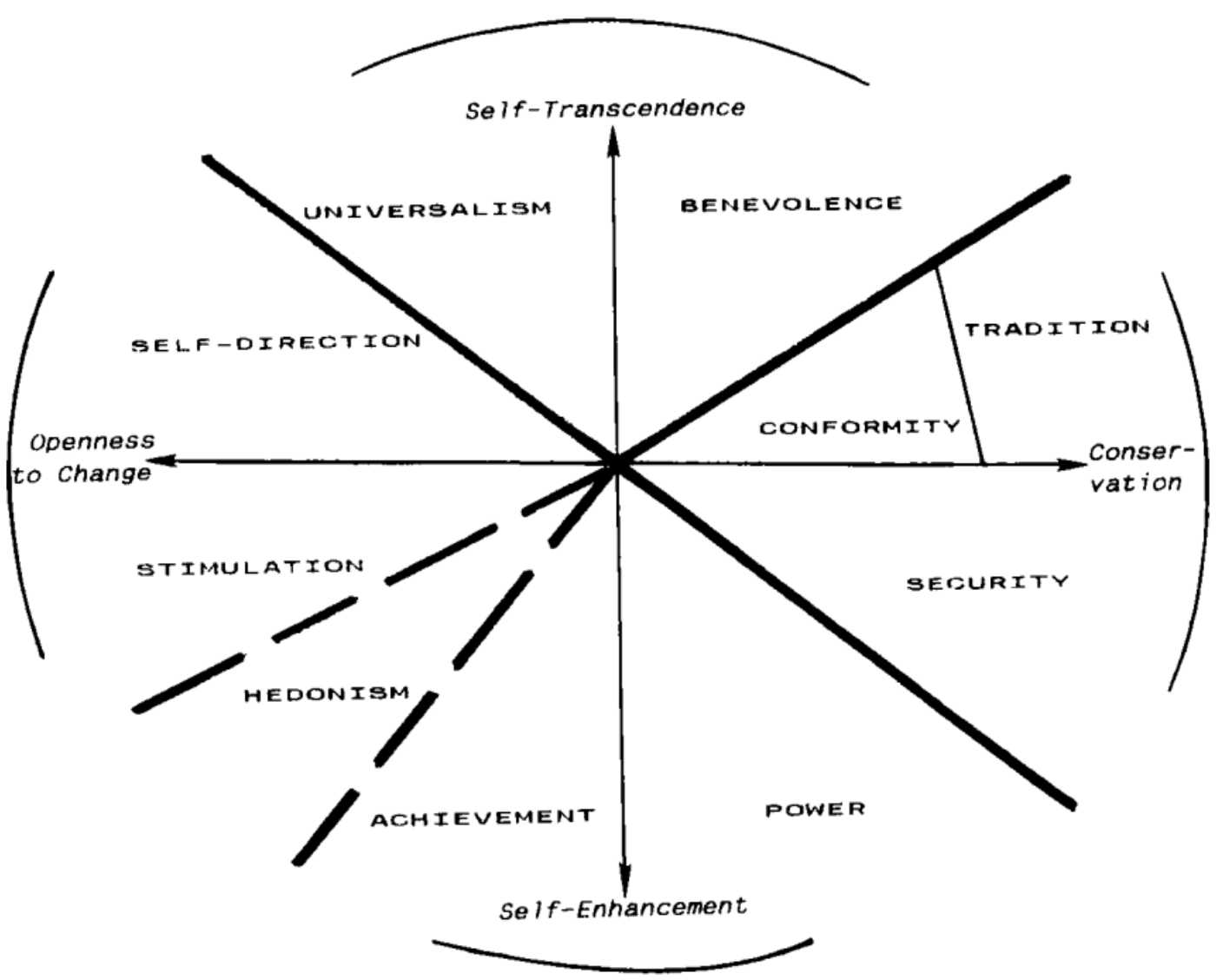

Figure 1

Theoretical model displaying the structure of values (Schwartz, 1992)

As demonstrated in Figure 1., the sections have been situated to display which values are similar, and thereby compatible with each other (Schwartz, 1992). While each of these values is present in all people, the relative strength of each value differs between individuals. Strongly held values tend to 'cluster' in one area of the circumplex; if a person strongly values, for example, 'universalism,' they are likely to also strongly value the adjacent values' benevolence' and 'self-direction.' Values on opposite ends of the circumplex, on the other hand, conflict. For instance, selftranscendent values and self-enhancement values are antithetical and therefore, incompatible. Explained by Dittmar, "serving other people's interests" is contradictory to "serving one's own interests" (2008, p. 79). If an individual attempts to hold both these values as equally important, they will feel internal conflict, detrimental to their wellbeing (Burroughs \& Rindfleisch, 2002). The relative strength 
of values is not permanent but can shift in response to changing life experiences. Within any shifts in values, the circumplex structure remains stable (Schwartz, 2011). For instance, growing importance on achievement will cause an increase in the adjacent values of power and hedonism, and a decrease in the opposite values of conformity and tradition.

A central theme of this thesis is examining how an individual's values shift and evolve according to their social contexts. Considerable research in this area has emerged within the theoretical framework Self Determination Theory, discussed in the following Section 2.4.

\subsection{Introducing self-determination theory}

Self-determination theory (SDT) is a psychological framework first developed by Ryan and Deci in 1985. It theorises that there are three fundamental human needs, which people instinctively strive for, which contribute to an individual's feelings of wellbeing (Ryan \& Deci, 2017). These three needs are:

Autonomy: Feeling ownership over one's own behaviour; that one's actions are a genuine expression of self. There is no sense of coercion into doing/endorsing something one does not truly want to.

Competence: a sense of "confidence and effectance in action" (Ryan \& Deci, 2017, p. 7), of feeling sufficiently challenged but also capable of overcoming challenges.

Relatedness: feeling connected with other people, caring for others and feeling cared for; a sense of belongingness with other individuals and with a community.

These three needs can be either supported or thwarted in any social context. An integral component of SDT is its imperative to "examine and compare social contexts in terms of their adequacy in supporting versus impairing human thriving" (Ryan \& Deci, 2017, p. 4). This premise acknowledges the propensity of humans to be influenced by their environment, which can either support or thwart the three basic needs and, therefore, human flourishing. Social contexts have an immediate 
effect on supporting or thwarting a person's experience of autonomy, competence and relatedness; but also cumulatively build to create more long term effects on wellbeing. Thus, social institutions which enable sustained thwarting of any or all of the basic human needs may have large-scale cultural impacts.

SDT aims to examine "factors that facilitate or undermine intrinsic motivation" (Ryan \& Deci, 2017, p. 124), according to the understanding that social contexts which support basic needs aid intrinsic motivation. Conversely, in contexts which thwart basic needs, individuals are more likely to adopt extrinsic orientations in attempt to repair needs. Any social context can thus be examined to determine how it supports or thwarts basic needs, and, following this, values. Intrinsic and extrinsic values are discussed in the following section.

\subsection{Intrinsic and extrinsic values}

Kasser brought Schwartz's theory of values and SDT together, arguing that they were compatible and would be mutually beneficial (2002a). The result emerged as Goal Contents Theory (GCT), a mini-theory within the framework of SDT. GCT examines how different goal orientations affect outcomes, such as environmental behaviour, materialism, and measures of wellbeing. Kasser explains, "a selfdetermination theory of values must recognise that some values are conducive to growthful, intrinsically motivated actions and others tend to prompt extrinsically motivated behaviours focussed on rewards and people's praise" (2002a, pp. 127128). While, as evident in the name, GCT speaks predominantly of goals rather than values, the two are closely linked; with an assessment of the importance of different life goals used as a way to empirically assess a person's relative importance of values (Kasser, 2019).

GCT is premised on the argument that "not all goals are created equal" (Ryan \& Deci, 2017, p. 272), in that some life goals contribute to basic psychological needs and wellbeing more than others. To demonstrate this, they categorised values as being either intrinsic or extrinsic. Intrinsic values, they state, are those which are important in and of themselves, including concepts such as personal growth and connection to nature. Intrinsic values more directly align with the three basic needs 
of SDT, reflected in consistent findings that individuals stronger in intrinsic values have a higher sense of wellbeing. Intrinsic values are also closely correlated with greater care for others and more environmentally responsible behaviour. A 2013 study of the attitudes of individuals with low carbon footprints found they did not necessarily feel strongly about climate change, but strongly associated with other intrinsic values such as social justice; which had significant downstream effects for their carbon footprint (Howell, 2013). Intrinsic values are also positively correlated with increased curiosity, creativity, productivity and compassion (Ryan \& Deci, 2017).

Extrinsic values are concerned with external signs of worth. They are motivated by expected approval or rewards, such as gaining wealth in order to be seen as successful, or looking a certain way in order to be complimented (Kasser, 2002a). Extrinsically based motivations tend "to conflict with the desire to help the world be a better place and to take care of others" (Kasser \& Kanner, 2004). Research has shown that people with stronger extrinsic values tend to have a higher carbon footprint and be less concerned about the environment (Hurst et al., 2013; Kasser, 2016; Kasser \& Kanner, 2004; Unanue et al., 2016). Individuals with stronger extrinsic values tend to be more self-serving rather than collaborative (Bauer et al., 2012). Research has found that extrinsic goals, even when attained, do not correlate with wellbeing (Deci \& Ryan, 2002).

There are certainly situations in which extrinsic values are useful or necessary. However, in this thesis I argue that, considering the research stated above, they are useful 'in moderation;' whereas modern Western culture has glorified extrinsic aspirations to the point that they are often detrimental to the wellbeing of individuals exposed to these ideas, and, moreover, implicated in the current crises that threaten the ecological balance of the planet (Kasser, 2016; Raworth, 2017). As stated by Kasser and Kanner, "we must all invest attention in material goals to survive, but there is a threshold along the continuum after which any further investment detracts from the ability to experience other aspects of life- such as relationships, aesthetic experiences, or the development of the body or the mind" (2004, p. 92). Current Western cultural messaging is often saturated with the glorification of extrinsic values such as fame, wealth and image (see Section 2.9). I thereby carried out this 
research under the understanding that it is necessary to limit such extrinsic incentives, and instead promote more intrinsic values, in order to work toward goals of mental and ecological wellbeing.

The values relevant to GCT are defined in Table 2 below:

\section{Table 2}

Goal Contents, Descriptions, and Sample Items (Grouzet et al., 2005, p. 802)

\begin{tabular}{ll}
\hline \multicolumn{1}{c}{ Goal contents } & \multicolumn{1}{c}{ Description } \\
\hline Affiliation & To have satisfying relationships with family and friends \\
Community feeling & To improve the world through activism or generativity \\
Conformity & To fit in with other people \\
Financial success & To experience much sensual pleasure \\
Hedonism & To look attractive in terms of body and clothing \\
Image & To feel healthy and free of illness \\
Physical health & To be famous, well-known, and admired \\
Popularity & To ensure bodily integrity and safety \\
Safety & To feel competent and autonomous \\
Self-acceptance & To search for spiritual or religious understanding \\
Spirituality & \\
\end{tabular}

Grouzet et al (2005) arranged these values into a structure, using data from 1854 students from 15 nations. Similarly to Schwartz's circumplex, it demonstrates the relative compatibility of goals. Goals which are close to each other are compatible, while those opposite each other conflict; as demonstrated on the following page in Figure 2: 


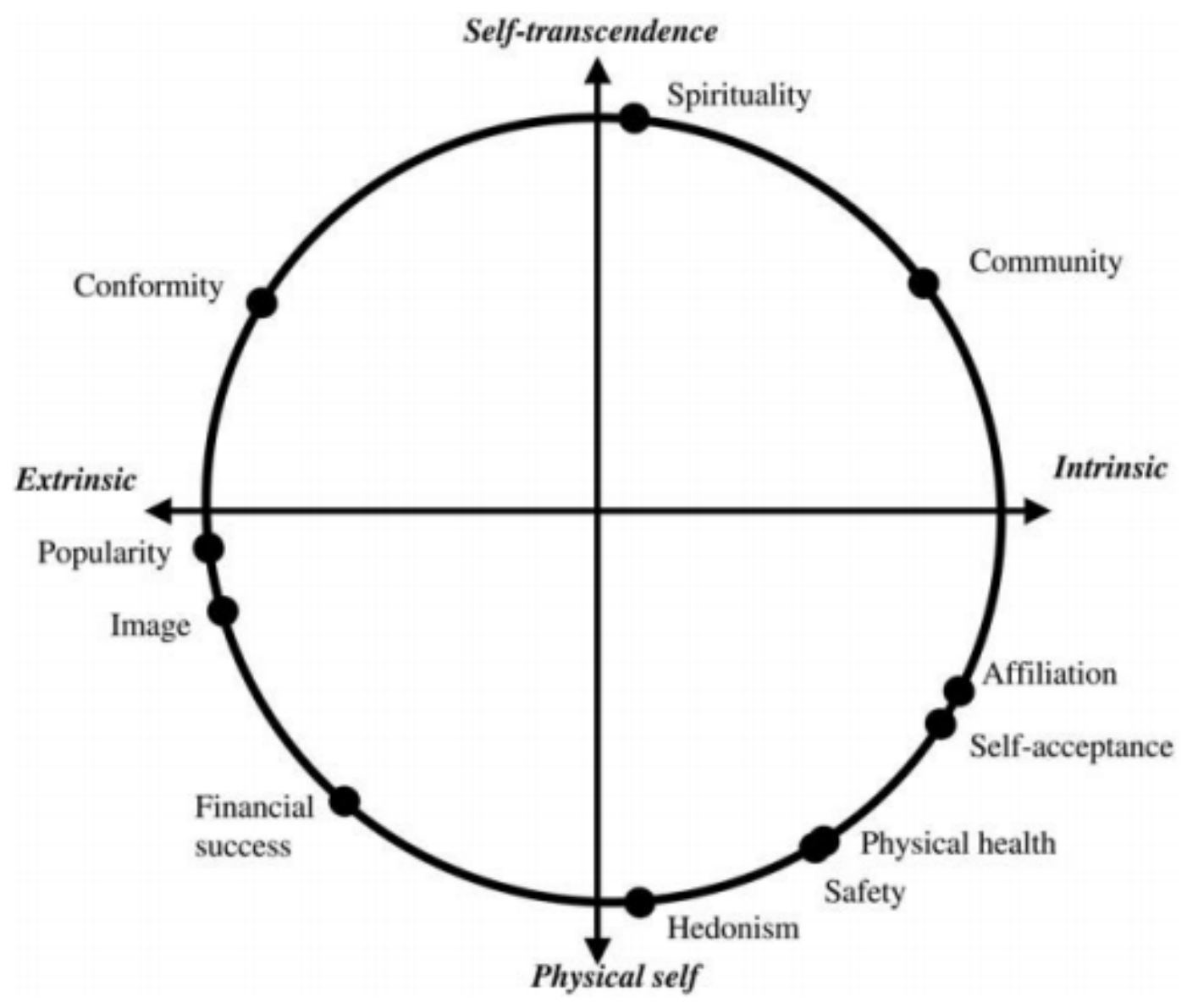

\section{Figure 2}

Circular representation of goals (Grouzet et al., 2005)

Grouzet et al.'s results largely showed universal consistency in the structure of goal contents. An exception was financial success, which the researchers found was situated as less extrinsic for participants from countries with lower average incomes than those from higher-earning nations. The overall average is displayed in Figure 2.2. The different outcome is likely due to the different interpretations of financial success; i.e. basic survival or welfare is an intrinsic need, whereas non-essential material consumption or status symbols is extrinsic. This difference demonstrates the importance, and nuance, of motivation rather than making blanket assumption around the categories themselves.

Further empirical research focusing more specifically on materialistic values placed materialism, as predicted, within the scope of extrinsic values, alongside power and achievement (Kasser, 2016). Although materialism comes under the umbrella of 
extrinsic values, they often appear in research as synonyms, with scales to measure the two (i.e. the Material Values Scale and Affirmation Index) used interchangeably (Hurst et al., 2013). Materialistic values are correlated with higher levels of social anxiety, lower relationship quality, less empathy, more competitiveness, and report overall lower levels of wellbeing (Kasser \& Kanner, 2004). A 2013 meta-analysis found significant negative correlation between materialistic values and proenvironmental attitudes and behaviours (Hurst et al., 2013).

Goal Contents Theory is useful in explaining why individuals adopt extrinsic values that tend to be counter to mental and ecological wellbeing. Kasser states: "individuals often give up their own internal locus of evaluation in order to obtain the love and affection of others, and thus hold introjected values which are based more on what others value than on what would facilitate actualisation of the true self" (2002a, p. 128). If an individual's need for relatedness is not being fulfilled, they may adopt extrinsic values in pursuit of social approval.

\subsection{Congruence between Schwartz and SDT}

Kasser argues that Schwartz's theory of values and SDT (which GCT sits within) are compatible and mutually complementary (2002a). Both Schwartz and SDT recognise an interconnected and dynamic system, and both have organised values/goals into a circumplex structure, with values/goals relatively compatible or conflicting with each other (see Figures 2.1 and 2.2). Both theories maintain that values can shift in adaptation to social contexts, and a strengthening of one values also strengthens those adjacent and weakens those opposite. There are further similarities within the arrangement of values: The Schwartz circumplex shows stimulation, self-direction, universalism and benevolence as compatible values which SDT similarly group together as intrinsic. On the opposite side of the Schwartz circumplex, power, achievement, and conformity/tradition are grouped together; values which, likewise, SDT groups as are extrinsic.

Schwartz's theory of values and SDT were developed separately, and, as argued by Kasser (2002a), some different terms exist for what appear to be the same underlying intentions. Schwartz described a need to seek out novelty and be in charge of one's 
own life as stimulation and self-direction, while SDT describes this as the basic human need of autonomy, or the value of self-acceptance. Schwartz describes a need to be connected with others as universalism and benevolence, which SDT names as the basic need for relatedness, or the value of community feeling. These examples demonstrate similarities that may not be immediately apparent.

On the other side of the coin, there are also examples of SDT and the Schwartz circumplex using the same terms to describe different concepts. For instance, Schwartz places 'conformity' close to 'benevolence,' interpreting it as a form of affiliation. Goal Contents, on the other hand, categorises conformity as an extrinsic pursuit concerned with approval from others. Further, Schwartz places' security' as opposite hedonism and adjacent to 'power;' while SDT theorists have interpreted it as safety, an intrinsic human need. While there are such differences between the two models, the overarching structure and contents broadly align. This thesis will predominantly reference SDT, with acknowledgement to Schwartz for his foundational work on values.

\subsection{Linking mental and ecological wellbeing through values}

Prevalent public discourse often states that responding to environmental issues necessitates personal sacrifice (Brown \& Kasser, 2005), which increases public resistance to changes toward a more sustainable society (Venhoeven et al., 2013). However, empirical research on values has found evidence that mental and ecological wellbeing are compatible. An orientation toward intrinsic values correlates with both mental wellbeing and environmentally responsible behaviour. For instance, Brown and Kasser (2005) found that happier people tended to live in more ecologically responsible ways, demonstrating both outcomes were predicted by stronger intrinsic values or participation in mindfulness practise. Venhoeven (2013) found that individuals who participate in environmentally responsible behaviour because they believe it is the right thing to do can see an increase in wellbeing. In a longitudinal study. Kasser and Ryan (2001) showed that progress toward nonmaterialistic goals, such as improving close relationships, increased wellbeing, whereas progress toward materialistic goals, e.g. gaining money or fame, did not. 
As evident in the values circumplex, materialistic values are opposite and conflicting with environmental values. If environmental values correlate with mental wellbeing, it would thus be expected that materialistic values negatively correlate with mental wellbeing. Evidence demonstrates this to be the case: For example, a study showed U.S adults who endorse materialistic values reported lower overall life satisfaction (Richins \& Dawson, 1992). Another study showed that students in Singapore with stronger materialistic value orientation reported lower levels of happiness and increased anxiety (Kasser \& Ahuvia, 2002). A study focussing on the relationship between intrinsic/extrinsic goals and wellbeing found a strong negative association between extrinsic goals and self-actualisation and vitality. A meta-analysis of 258 samples revealed an overall negative correlation between materialism and wellbeing (Dittmar et al., 2014).

Considering the above evidence, it follows that mental and ecological wellbeing are compatible; and the popularly perceived trade-off between the two does not necessarily exist. $\mathrm{Ku}$ suggests that the compatibility between mental and ecological wellbeing exists because "intrinsically-oriented individuals may be less likely to engage in harmful environmental behaviour because their goals, unlike those in individuals driven by materialism, rest not on the possession of material goods, which impact the environment, but on their own personal growth and relationships with others" (2014, p. 473). Venhoeven explains, "shifting one's focus from the pursuit of materialistic things like money and products, to the pursuit of nonmaterialistic things like close relationships, personal growth and finding a sense of meaning in life is not only more pro-environmental, but it also contributes to eudemonic wellbeing" (2013, p. 1378). This argument aligns with the basic needs described in self-determination theory (see Section 2.3). Goals pertaining to "personal growth" are intrinsic and related to the basic human needs stated by SDT as necessary for human flourishing.

With evidence of links between intrinsic values, greater wellbeing and more environmentally responsible behaviour, why then do extrinsic goals hold such global prominence? 


\title{
2.8 Situational value activation
}

Kasser emphasises that people are not categorically 'materialistic' or 'nonmaterialistic,' but that materialistic aspirations can develop in any person, depending on their social-environmental context. He maintains:

\begin{abstract}
"Although it may be easy to believe that research on materialism mostly concerns the study of materialistic individuals who strongly prioritise such aims in life, a more useful, and, I think, more accurate attitude is to recognise that all of us have materialistic tendencies. These tendencies can be activated in a particular moment, enhanced when we see them modelled in society, and brought on by temporary or chronic feelings of threat or insecurity. As such, researchers need to ask not only, "Who is materialistic?" but also "When are people materialistic?" " (Kasser, 2016, p. 506)
\end{abstract}

A significant body of research has shown the capacity for social contexts to activate different values in a person, affecting immediate behaviour. As described by Kim, 'exposure to media filled with lavish consumption might spontaneously activate materialistic thoughts" (2013, p. 770). This hypothesis has been demonstrated through a multitude of experiments in 'priming,' in which participants are subconsciously exposed to specific concepts in order to determine how they affect cognitive processes (Dehaene et al., 1998). A significant body of research has shown that priming individuals with the concept of money activates the value of 'wealth' in the values circumplex and, in association, activates associated extrinsic behaviours. A meta-analysis of 246 experiments showed of money priming to have an overall significant effect on behaviour (Lodder et al., 2019). These experiments show the extent to which people are affected by their immediate environmental context. For instance, Vohs (2006) demonstrated that after being primed with money, participants (compared to control groups) were less helpful to fellow participants, placed chairs further away from co-participants, and preferred spending leisure time alone. In another experiment, executives who solved riddles which included the financial words "profit" and "growth" subsequently acted less empathetically to colleagues than those in a control group (Molinsky et al., 2012). Bauer (2012) showed participants 24 images of either luxury goods or neutral images. The subsequent questionnaire, presented as unrelated, showed that those who viewed the luxury 
items were significantly more depressed and anxious, and less interested in social activities.

Similarly, priming a particular identity means that individuals may enact a behaviour associated with that identity. Bauer (2012) found that naming a computer task as a "Consumer Reaction Study" as opposed to a "Citizen Reaction Study" had the effect of making participants approach materialistically themed words faster in a computer task. She also found, in a hypothetical water shortage activity, that when participants were named 'consumers' they stated lower feelings of responsibility to conserve water compared with participants who were referred to as 'citizens.' These results prove concerning when combined with a study from a real-life context. Shrubsole (2012)shows the increasing pervasiveness of the term 'consumer' in real-world settings. In a study of 5 million English language books and two British newspapers, the term 'consumer' overtook the term 'citizens' in frequency of use in the 1970s. A study of tabloids showing similar trends states concerns around mainstream media encouraging identities defined by what one consumes, rather than a citizen with full democratic rights and responsibilities (Rooney, 2000).

Further studies show that priming nature may have the opposite effect, activating more intrinsic values. Weinstein (2009) measured participants intrinsic and extrinsic aspirations, then exposed them to a slideshow, with an audial guided imagery script, of either nature or human-made infrastructure. He found that participants who viewed slides of nature subsequently rated intrinsic aspirations as more important to them, and decreased their importance of extrinsic aspirations. Participants who viewed slides of human-made infrastructure increased in extrinsic aspirations, with unchanged intrinsic aspirations. Zelenski (2015) found that participants who watched a twelve-minute video of nature, as opposed to a video of a built environment, acted significantly more sustainably in a simulated fishing game.

Moldes and $\mathrm{Ku}$ (2020) completed a meta-analysis on situational materialism and wellbeing and found empirical evidence that materialistic cues cause lower wellbeing at both an individual and societal level (societal wellbeing included environmental measures). If this is true at an experimental level, it is feasible that, considering the consumerist nature of current Western society, individual and 
societal wellbeing are being eroded at a significantly larger scale. Broader trends in terms of values will be discussed in the following section.

\subsection{Extrinsic influences}

Many cultural practises around the world have specifically criticised extrinsic pursuits. Religious philosophy, Buddhism, Hinduism, Islam, Judaism and Christianity all warn against pursuing excessive material wealth (Belk, 1983). The explicit practices of other cultures indicate recognition of both the human capacity for extrinsic values such as self-promotion, wealth and status, and the deliberate effort to suppress them in pursuit of a well-functioning society.

However, in current Western neoliberal society, such extrinsic values such as wealth, fame and image are central to often celebrated as central societal values. As stated by Kasser and Kanner, "never before in humankind's history has our drive toward materialism and consumption been offered such opportunity for expression and satisfaction" (2004, p. 11), a trend which has only continued to grow in the years since their words were published.

GCT attributes this largely to the advertising industry, which directly promotes the values of consumption. According to Gonick and Kasser (2018), the level of television advertising to children was the most likely variable to predict stronger hierarchical values of countries (such as authority, status and wealth). Egalitarian nations (which place higher value on equality and social justice) have regulations in place to shelter children from advertising. In contrast, hierarchical nations "put advertisers' interests first" with less regulations, thus instilling extrinsic values throughout generations (Gonick \& Kasser, 2018, p. 128).

A multitude of researchers state concerns around the pervasiveness of materialistic influences in modern Western society (Hamilton, 2005; Jackson, 2017; Kasser, 2016). Richins states, "Materialism is perpetuated in an individual because it is reinforced" (2017, p. 488). Seeing frequent messaging stating, subliminally or otherwise, the importance of materialistic pursuits may prime the associated values. 
When primed frequently enough, there is likely to be a more permanent effect on the values' comparative strength.

According to SDT, both mental and ecological wellbeing would benefit from social contexts which promote more intrinsic values. Yet, economic goals are regularly prioritised (Hamilton, 2004; Jackson, 2017; Moldes \& Ku, 2020; Raworth, 2017), meaning cultural values are often dominated by extrinsic ideals of wealth and consumption. As Kasser and Kanner state, "from the time they are born, people receive implicit and explicit messages endorsing the importance of money and possessions" (2004, p. 16). Exposure to consumerist messages creates "enormous pressures to conform to the beliefs and values of this culture" (Kasser \& Kanner, 2004, pp. 12-13). This indicates firstly that consumer culture is constructivist, and therefore changeable, and secondly that sociocultural pressures have an important influence in shaping individual values.

\subsection{Cultural values and the scope for change}

There is no clear consensus on how exactly to define cultural values. While researchers have advocated for a distinction between individual and cultural values, they have simultaneously measured cultural values by aggregating individual values and finding the average (Schwartz, 2011). Schwartz argues that cultural values are prescriptive, describing them as "the normative value emphases that underlie and justify the functioning of societal institutions" (2011, p. 314). Under this definition, cultural values form a bracket of acceptability for individual values. This understanding bears a resemblance to the concept of an "Overton window," which signifies the spectrum of what is politically acceptable (Boyd, 2016). The Overton window shifts over time, as ideas at one time thought politically inconceivable (for example, ending the slave trade) shift in public consciousness until eventually becoming accepted and normalised. The same is plausibly true of cultural values, and has been coined by Monbiot as a "values rachet" (2014). Thus, while perhaps cultural values are a sum of the individual parts, they also in turn influence individual values by determining what is within the bounds of social acceptability. Reciprocally, individuals at the extremes of what is considered acceptable may influence cultural values, by pushing the boundaries and exposing or normalising 
new ideas or behaviours. Therefore, a close and dynamic relationship exists between cultural and individual values, in that both are malleable, and each influences the other.

According to research, in recent decades a shift has occurred toward a cultural prioritisation of extrinsic values such as wealth, status, image and fame (Dittmar, 2008; Gonick \& Kasser, 2018; Kasser \& Linn, 2016; Monbiot, 2014). This shift has grown to the point that, as Ryan stated in his foreword, "selfishness and materialism are no longer being seen as moral problems, but as cardinal goals of life" (Kasser, 2002 b, p. ix). Studies show that in many parts of the world, populations are increasingly prioritising extrinsic goals. A Turkish study found generational differences in increased self-enhancement (extrinsic) values and decreased selftranscendent (intrinsic) values across two decades (Marcus et al., 2017). In the United States, students are reporting increased importance on financial achievement and less on finding a philosophy of life (Twenge, 2017), and studies of values over three generations showed adolescents growing less concerned with others, community or the environment (Twenge et al., 2012). Concurrently, in the United States, the 'luxury goods' market has grown four times the rate of the overall economy, exemplifying an increase in expectations of material needs (Hamilton, 2005). The cultural normalisation of stronger relative extrinsic values may contribute to the increasingly critical overstepping of ecological limits. Nevertheless, it demonstrates the malleability of values, and the capacity for them to shift according to sociocultural context.

Longitudinal studies have shown the capacity for individuals to shift toward more intrinsic values. For instance, Lekes et al. (2012) instructed students to write about intrinsic values, and undertake weekly reflections on these values for a month. The study showed that the more engaged in the exercise the students were, the more their relative intrinsic aspirations increased, and the greater their reported levels of wellbeing improved. Lambert et al. (2009) demonstrated a shift away from materialistic values when participants undertook a gratitude reflection. Brown and Kasser (2005) discussed mindfulness as a link between subjective wellbeing, ecological behaviour and intrinsic values. This argument was expanded by Brown et al. (2009) to show that individuals who attended a four-week mindfulness meditation 
practise subsequently rated higher levels of subjective wellbeing and less financial desires, theorised by the authors as being related to the adoption of stronger intrinsic values. In another study, Kasser et al. (2014) organised three 3-hour group sessions critiquing consumer culture and reflecting on values. Compared to a no-treatment control group, the participants decreased in measures of materialism and increased in self-esteem, with both trends still evident ten months later.

According to Schwartz, "Individuals adapt their value priorities to be compatible with the opportunities and constraints they confront" (2011, p. 311). While the above examples consider the adaptation of individual values as opposed to cultural, they nevertheless demonstrate how a small shift in an environmental context can have powerful effects. Considering the extent to which messages of materialism permeate Western culture, alternate messaging that instead centres on intrinsic values may have the potential to shift cultural values towards a more intrinsic orientation, thereby improving mental and ecological wellbeing.

\subsection{Conclusion}

Values underpin attitudes, behaviours and goals. Further, research shows that some values have strong associations with both mental wellbeing and environmentally responsible behaviour. This association indicates that responding to climate change may find solutions, and simultaneously greater levels of mental wellbeing may be achieved, through a cultural reprioritisation of values.

Values theory has foundations in Schwartz's research, mapping universal values and their relations to each other. Self Determination Theory states that there are three basic human needs critical to human flourishing, which socio-environmental environments can either support or thwart. Combining Schwartz and Self Determination Theory, Goal Contents Theory holds that some values contribute to basic human needs more than others. Values that are important for their own sake, such as close personal relationships or involvement in causes bigger than oneself, are categorised as intrinsic, and are correlated with higher levels of mental wellbeing and a lower carbon footprint. Values which are reliant on external rewards to measure success, such as fame of symbols of wealth, are extrinsic. Extrinsic values 
tend to predict lower levels of wellbeing and a higher carbon footprint. If a person's basic human needs are thwarted, they are likely to take up extrinsic aspirations in an attempt to fulfil their needs.

Values shift at both a cultural and individual level, in response to socioenvironmental signals. In recent decades, Western cultural values have become overall more extrinsic, to the detriment of people and planet; likely due to the saturation of consumerist messaging in modern Western societies. There is thus a conflict between predominant cultural incentives toward materialism, and goals regarding mental wellbeing and responding to climate change. A values-based approach illuminates this disjuncture, and, further, illuminates a pathway toward solutions. 


\section{Instagram}

"The unintended consequences of a network when it grows to a billion or 2 billion people ... it literally changes your relationship with society, with each other"

-Sean Parker, first President of Facebook (Allen, 2017)

\subsection{Infrastructural context}

As described above, the infrastructure of social environments influences cultural values; as people "inherit, inevitably, the prescriptions and proscriptions of our social institutions" (Schwartz, 1986, p. 29). Ryan and Deci argue that "pervasive cultural norms or economic structures represent "invisible" or implicit values, constraints and affordances, which are then reflected in a more proximal social conditions and conveyed by socialising agents from parents and teachers to cultural messengers such as religious leaders, politicians and celebrities" (2017, p. 562). Instagram has emerged as a powerful platform for young people to absorb, and reflect, its cultural norms. The implicit values represented on the platform have a global reach, signifying the societally accepted boundaries of individual values.

Within social media, like any institution, are often unacknowledged power structures. As Srinivasan critiques, "it is assumed that "connection" is better, and that somehow with greater connectivity equality arises. Little is spoken about who owns these connections, who creates and manages the architectures of connectivity, and whose underlying epistemology drives the structure and network of connectivity" (2017, p. 13). Social media platforms export the ideologies and values of the culture they are developed within to the rest of the world in which they are used. Instagram was developed in the United States, one of the most ideologically neoliberal nations in the world (Kasser \& Ryan, 1996). The uptake of Instagram around the world is also an uptake of a platform rooted in the ontology of Silicon Valley, with pervasive global influence and few options for regulatory control ( $\mathrm{Wu}$, 2019). When any person in the world uses Instagram, they use a platform designed and regulated according to neoliberal United States values. 


\subsection{Instagram: An overview}

The social media platform Instagram, a free-to-use application for sharing filterenhanced images, has rapidly become a pervasive part of life for a large proportion of the world's population. Launched in 2010 within the burgeoning technology hub of Silicon Valley, California, Instagram rapidly grew to global prominence, reaching a billion active users within a decade (Statista, 2020). In 2012, Instagram became part of one of the most powerful global corporations when it was purchased by Facebook.

Instagram has become widely recognised in the cultural landscape, to the extent that galleries and tourist attractions have emerged specifically targeted toward providing visitors with content to create attractive Instagram images (Pardes, 2017; Phan \& Macias, 2018). The modern catch-phrase "Do it for the "Gram" is referenced in pop culture and academia, describing the motivation to participate in an activity primarily in order to post about it on Instagram. Instagram has been particularly popular with young people, with $43 \%$ of users aged under 25 (Statista, 2020). This age group is particularly sensitive to outside influences, as they are in a critical phase of forming identity and are neurologically still developing (Sapolsky, 2017). The global prominence and young age of users make Instagram a powerful force in shaping global culture and values.

A study by Sheldon and Bryant (2016) asked 239 undergraduates why they use Instagram. The top category was surveillance/knowledge of others, such as interacting with friends and seeing their visual updates. Documenting their lives, events and surroundings was the second most popular motive, and 'coolness,' including to become popular and self-promote, was third. However, this study does not go into detail on the nature of the content they see on Instagram, and how it may reflect/influence values. For instance, under 'surveillance,' someone who sees their friends' polished luxurious lifestyles may have a different experience than someone who views images of friends enjoying nature or participating in community/volunteer work. Similarly varied outcomes are possible for the second most popular motivation, self-documentation. The third stated motivation, 
'coolness,' is an extrinsic orientation, as it is concerned with how one is seen by others and thus dependent on external validation.

Research on social media has examined a range of uses and effects of online platforms, with nuanced and, at times, contradictory findings. These were collated in a meta-analysis of 56 studies on the psychological or sociocultural effects of using social media, which found that the negative outcomes of using the platform outweighed the positives (Conger \& Singg, 2020). Adverse outcomes were especially pertinent for moderate to heavy users of social media. These outcomes were predominantly related to mental impacts of FOMO (Fear of Missing Out), objectification, social media addiction, and, lastly, social comparison; which is of particular relevance to this thesis and expanded upon in Section 3.3.

\subsection{Social Comparison}

The most prevalent Instagram activity is viewing content posted by others (de Vries \& Kühne, 2015). Each user chooses which profiles they follow, thus personalising the content they see. Users can follow friends and family, celebrities and a vast array of personal interests such as nature photographers, recipes, fashion or poetry. Users scroll through a 'feed' displaying images posted by these profiles, interspersed with paid advertisements specifically tailored to the users' interests. Significant attention in recent years has examined the effects of viewing Instagram images through the theory of social comparison.

Established by Festinger (1954), the theory of social comparison states that it is an innate human tendency to compare oneself to others in order to be inspired and check one's opinions and abilities are in line with others. However, literature has demonstrated how an overload of upward social comparison, comparing oneself with those viewed as 'better,' can make people feel personally inadequate. This experience appears to be prevalent on Instagram. For example, Ho and Ito describe how Instagram profiles tend to be an idealised self-perception which is "more socially desirable and glamorous than reality, and thus are biased representations with potential impact on viewers' psychological states" (2017, p. 1359). Similarly, de Vries and Kühne argue that through exposure to Instagram's idealised 
representations of peers' lives, social media users are "more inclined to believe that others are having better lives than they are" (2015, p. 218). This argument echoes what Hamilton describes as a "ratcheting up (of expectations) ... through presenting opulence as normal and attainable" (2005, p. 9), with people seeing ever-better standards of living and therefore perpetually feeling personally deprived.

Exposure to idealised content may have a direct impact on materialistic behaviour. Richins suggests that young people can "feel inferior about their own appearance and possessions at a time when they're especially vulnerable to these kinds of comparisons... (this) may lead to negative self-feelings and encourage materialistic behaviour to repair those feelings" (2017, p. 485). Mandel et al (2006) similarly found that upward social comparison increased participants' desire for luxury goods. These findings align with SDT: The need to feel competent is not being met, and therefore, other mechanisms are employed in an attempt to fill the gap. It further aligns with Kasser and Kanner's research showing feelings of low self-esteem predicate higher levels of materialism, and thereby less environmentally responsible behaviour (2004).

The materialistic incentives arising from negative perceptions of self may be exacerbated by exposure to messaging which links concepts of happiness, affluence and attractiveness. Ho and Ito found that social media profiles tend to display both a perception that others are "happier and having better lives" than the viewer, and at the same time portraying "idealised images of affluence and attractiveness" (2019, p. 1360). The combination of visible themes of happiness, affluence and attractiveness sends the signal to viewers that the three concepts correlate, and that if they can improve their attractiveness or make more money, they can be as happy as the people in the images they see. On the contrary, as discussed in Section 2.6, concerns with wealth and image are detrimental to wellbeing.

\subsection{Arguments of self-commodification through Instagram}

A growing body of research argues that social media is an extension of consumer culture, in that it turns the social media users themselves into a commodity (Khamis et al., 2017; Page, 2012; Smith, 2018). Page states that social media platforms create 
a framework for the "construction of identity as a product to be consumed by others, and an interaction which treats the audience as an aggregated fan base to be developed and maintained in order to achieve social or economic benefit" (2012, p. 182). The carefully curated virtual lives are consumed by others either through social capital measured in 'likes' and 'follows;' or for the most popular Instagrammers, financially, through product placement in their images.

Smith similarly describes the commodification of the social media user, with life experiences increasingly being constructed for social media gratification. He states that tourism is becoming increasingly occupied with "a search for the perfect Instagram image, itself a representation of experience as well as a commodity that can be exchanged online for 'likes'.” (2018, p. 175). When motivated by social media, an experience of the moment is lessened, to instead become a preoccupation with representing that moment visually in order to gain gratification from others.

The motivation of gaining attention and gratification from others (in the form of likes and follows) closely links with extrinsic aspirations of status, image and social recognition. Smith suggests that when photographing landscape images, "in both the process of photographing it and in posting that image to Instagram the landscape is transformed into a commodity" (p.183). In pursuit of Instagram success, users are incentivised to purposefully seek out photogenic locations, resulting in "only travelling for [one's] Instagram" (2018, p.183). In this situation, the experience of travelling is predominantly motivated by the will to enhance one's online selfrepresentation. The landscape is not enjoyed for its own sake, but as a means to an end; something to be traded online for increased social recognition. Focusing attention on these extrinsic pursuits is simultaneously moving attention away from the intrinsic pursuits that would be more conducive to satisfying basic human needs. While these studies are theoretical and do not reach empirical conclusions, the discussions of commodification on Instagram indicates ways the platform may be particularly conducive to exacerbating extrinsic orientations. 


\section{Objectives and research questions}

\subsection{Research objectives}

Considering the urgency of social change required to avoid catastrophic climate change and the central role of values in environmentally responsible behaviour, the institutions shaping cultural and individual values deserve more scholarly attention. In undertaking this research, I wish to examine how social contexts influence values, and by association, mental and ecological wellbeing. Research on values illuminates an opportunity for a 'win-win'- for an improved state of being for people and the planet. Despite widespread empirical knowledge of the benefits of pursuing intrinsic aspirations, studies show a trend toward the opposite trajectory, with increases in extrinsic or materialistic values. This, as discussed by numerous values researchers (Gonick \& Kasser, 2018; Hamilton, 2004; Jackson, 2017; Kasser \& Kanner, 2004; Richins, 2017), is likely due to the saturation of extrinsic-based messaging in the modern Western social environments. Research has looked at the extent to which people are influenced by their environment, including by materialistic messaging in modern Western culture. Further, a large body of research has emerged looking at varied effects of social media, including Instagram. However, there remains a gap in bringing these two lines of research together to see how Instagram may be affecting intrinsic or extrinsic values.

Both Kasser (2016) and Schwartz (2011) recommend examining different media environments to ascertain how they may be influencing values. In this thesis, I follow this recommendation, focussing on the experiences of users of the social media platform Instagram. Instagram, a global social media platform with over a billion users, does not appear to have been examined in terms of how it may be influencing values. There is little existing qualitative research on the user's experiences of Instagram through a values lens. Do they experience pressure toward materialistic goals? What are the values they see promoted on Instagram? Does the platform support or thwart basic human needs? As each user sees uniquely tailored 
content, research investigating these questions cannot be representative; yet it can offer valuable insights into personal experiences.

\subsection{Research questions}

My research questions are as follows:

1. How do participants' experiences of Instagram demonstrate incentives toward intrinsic or extrinsic values?

2. What do these findings mean for mental and ecological wellbeing? 


\section{Methodology}

\subsection{Positionality statement / reflexivity}

This research is situated within, and examining, modern Western culture. While the research takes a critical lens of mainstream Western values and institutions, nevertheless, my analysis is shaped by my experience growing up, and still living, embedded within the predominantly Western constructs of colonised Aotearoa New Zealand. As stated by Stanley and Wise, "Our consciousness is always the medium through which the research occurs, there is no method or technique of doing research other than through the medium of the researcher" (1983, p. 157). Therefore, my upbringing, my epistemology, and the culture surrounding me necessarily influences the nature and results of this research. These influences emerge in multiple ways: by nesting this study within a thesis in Environmental Studies, by seeing the world through the lens of a climate activist, and by my unique life experiences and ideas I have been exposed to. My identity shapes the research directly through data collection; in terms of whom I have access to for interviews, which questions it occurs to me to ask, and which elements of the conversation I find interesting and worthy of pursuing within the conversation and in the analysis afterwards. Interview participants were aware that the research was under the banner of Environmental Studies, and that could have led to some social desirability bias. For example, it could be assumed that environmentalists frown upon materialistic priorities, and that participants answered questions with that in mind. Results may therefore have been different had the same questions been asked by someone studying Instagram toward a degree of a different nature. Such influences are unavoidable yet are not necessarily a weakness: According to social constructionism, it is interactions which shape the research process (Charmaz, 2008).

Recognising that research is influenced by the subjectivity and values of the researcher, this influence needs to be made explicit. This is achieved through reflexivity: awareness and acknowledgement of the effect of the researcher on the research (McCabe \& Holmes, 2009). Reflexivity is a tool to identify researcher bias, 
to control/limit their influence on the research, and thereby ensure validity in qualitative research.

In accordance with the theories described below of social constructionism and critical emancipation, this research does not pretend to be value-free. In analysing this topic, my overarching aim is always concerning how catastrophic climate change can be avoided. The evidence showing a consistent correlation between intrinsic values and environmentally responsible behaviour leads me to the view that intrinsic values should be more incentivised by societal structures, and that global pressures encouraging extrinsic values should be minimised. This research reflects that view.

Concerning my own experience with Instagram, I am an active user, though I use it infrequently. I predominantly follow content portraying friends, activism, street art and food. I post content including myself, nature, friends, activism, travel and food.

\subsection{Epistemology: Social constructionism}

This research primarily takes the view of social constructionism, in which "the world is constructed by human beings as they interact and engage in interpretation" (O'Leary, 2014, p. 7). Social constructionism argues that there is no objective reality, and, therefore, no objective right or wrong. Each person's ontology is shaped by their own unique experiences, which in turn shapes the researcher's output. Research findings are thus contextualised within the ontologies of the researcher and the participants, and formed out of the interactions between them (Charmaz, 2008).

According to Silverman (2011), the constructionist view also allows conventional power relationships to be questioned. If there is objective truth, that means the holder of the knowledge is 'right', the other is 'wrong,' and within that lie implications that whoever is 'right' is superior to whoever is "wrong." However, within the theory of constructionism, different perceptions of reality are equally valid. Therefore, in regard to my positionality statement above, I acknowledge that others may have different and equally valid views. In my objective to understand participants' experiences of Instagram, I recognise that each person's perspectives are valid and 
reflective of their own ontology. The research findings are formed from the discussions between myself and the participants, and therefore I acknowledge that others examining the same issues may reach differing conclusions.

\subsection{Critical Emancipation}

This research engages the social constructivist theory of critical emancipation. Critical emancipation "refers to fundamental or revolutionary changes in current thinking, practices, conditions or institutions that can free people from the constructs of dominant social structures that often limit self-development and selfdetermination" (O'Leary, 2014, p. 192). This theory does not pretend to be politically neutral or value-free. An aim of critical emancipation is "to unmask the dominant forces that shape our view of the world" as it "attempts to contextualise the current situation in a larger socio-historic framework that offers, and encourages others to engage in, critical reflection" (O’Leary, 2014, p. 175). Considering the scale and urgency of the current ecological crises, it is now a "critical time for emancipatory social science to examine the opportunities, barriers, and possible trajectories of the climate movement to serve as a catalyst for positive transformation" (Stuart et al., 2020b, pp. 435-436).

In applying critical emancipation to this thesis, my aim is to uncover daily, largely unquestioned practises which shape our values and thereby our environment. Through this thesis, I aim to begin opening discussions on the potential conflict between the social media model and both mental and ecological wellbeing.

\subsection{Research methods}

In this thesis, I used qualitative research methods. According to Corbin and Strauss (2008), qualitative researchers are particularly drawn to creativity, finding order in disorder, and an acceptance of no final "correct" output. Qualitative methods allow more open-ended discovery and "the opportunity to connect with (participants) at a human level" (Corbin \& Strauss, 2008, p. 13). I chose to employ qualitative research methods as they align with the social constructivist epistemology, and allow a greater depth of understanding of user's experiences of Instagram. 


\subsection{Ethics}

Ethics approval was given by the Victoria University of Wellington Human Ethics Committee (application number 0000027774) for all data collection. All interviewees viewed and signed an information and consent form (see Appendix A).

\subsection{Interviews}

Eyles and Smith describe interviews as "a conversation with a purpose" (1988, p. 5). They are not designed to be representative (Tashakkori, 1998), nor replicated (O'Leary, 2014), but instead allow an in-depth examination of a person's experience on a specific topic. In this thesis, qualitative data was gathered through semistructured interviews. The only requirement for eligibility as a participant was being an active, or previously active, user of Instagram. However, I also made efforts to recruit participants who were relatively young, and female, as this is the primary demographic on Instagram (Sherlock \& Wagstaff, 2019), and the demographic which experiences the most negative impacts (RSPH, 2017). I used snowball sampling to find participants, predominantly successfully recruited through 'friends of friends.'

In total, I interviewed thirteen participants. Ten live within the Wellington region and three in Auckland. Twelve participants identified as female and one as male. Interviews ranged from forty-one minutes to two hours and thirteen minutes. Twelve participants were currently active on Instagram, with one previously active. Their use of Instagram ranged from aspiring 'influencers,' who use Instagram for several hours each day and post images related to fashion and self-promotion, to those who use the platform for activism and movement-building, to those who use it recreationally and follow a wide range of content.

Four interviews were one-on-one, and there were two group interviews of five participants each (one of the group interviewees, who had further experiences to share concerning Instagram, was later also interviewed independently, thus is counted twice). All interviewees signed a consent form. Time and location of 
interviews were organised at the convenience of the participants. Participants were assured they would be given pseudonyms in the final thesis, allowing them to feel they could speak more freely. The interviews were semi-structured and conversational in order to make participants feel more comfortable and allow the conversation to move organically, with flexibility to discuss subjects I had not preconsidered. The full interview schedule, which provided the basis for discussions, is available in Appendix C.

\subsection{List of Participants}

Note: All names are pseudonyms.

\section{One-on-one Interviews:}

Billie Uses Instagram forty minutes per day on average. Previously used it for four hours per day, when aspiring to be an Instagram influencer. Posts content including self, fashion, travel, fitness. Follows content including friends, celebrities, food, health, fitness, travel, fashion.

Georgia Uses Instagram less than daily. Posts predominantly activism content. Follows content including friends and activism.

Amy Uses Instagram on average two hours per day. Posts content including the self, friends, travel. Follows content including friends, celebrities, food, environmentalism.

Laura Uses Instagram on average one hour per day. Posts content including self, friends, travelling, food. Follows content including friends, fitness, food. Previously followed a lot of celebrities but found it detrimental to her mental health.

\section{Group Interview One:}

Jen, Megan, Caro, Kate and Krista: all female aged between 17-18. Post content related to activism on public profiles, thought also have private 
profiles to post personal posts related to self, daily events, friends.

Collectively follow content including friends, politics and activism.

\section{Group Interview Two:}

Lisa, Emily, Dan, Anna, Billie (Billie was also interviewed individually and is listed above). Four females and one male aged 20-26. Post content including nature, travelling and the self. Collectively follow content including food, beauty, friends, sports, education, tattoos, fashion, activists, and news.

\subsection{Data Analysis}

I examined the data collected through the interviews through grounded theory (Glaser \& Strauss, 1967). Grounded theory is, as the name suggests, a method of creating theory 'from the ground up.' It is a way of analysing data without preconception, finding themes according to participants' contributions. Grounded theory goes beyond "what is directly observable and explicitly stated" to uncover more subtle findings by repeatedly going through the data to find themes and patterns (Charmaz, 2008, p. 403). Concurrently, the findings are related back to the literature in order for it to inform emergent understandings (O'Leary, 2014). From this, a new theory may be generated (the original purpose, hence the name), or the researcher may use grounded theory techniques to find patterns and map themes. According to Corbin \& Strauss (2008), either approach is valid, so long as it is made clear what the researcher is aiming to do. Within the scope of this research, my aim was to discover patterns and themes as opposed to generate new theory.

To analyse the data through grounded theory, firstly I transcribed the audio from the interviews. The transcriptions were then coded and physically 'cut and pasted' into general themes. The arising themes, through repeatedly returning to literature, evolved and solidified, eventually becoming the sub-headings within the wider headings of the three chapters of analysis. While patterns and themes arose organically, each one had elements of either relatedness, competence or autonomy; the three basic needs of Self Determination Theory. Each of these needs forms a chapter of the final analysis. 
An example of how data evolved into themes is demonstrated below in Table 3:

\section{Table 3}

Methodological example of coding process

\begin{tabular}{|l|l|l|l|}
\hline $\begin{array}{l}\text { Quote taken from } \\
\text { transcript }\end{array}$ & Code & $\begin{array}{l}\text { Final sub-heading } \\
\text { human need }\end{array}$ \\
\hline $\begin{array}{l}\text { "I feel like-especially } \\
\text { like dining out, kind of } \\
\text { for me, it's like- I'd } \\
\text { rather go to this } \\
\text { restaurant over this one } \\
\text { because it's aesthetic, } \\
\text { you know? Like even if } \\
\text { it's not as good food. } \\
\text { offline life }\end{array}$ & $\begin{array}{l}\text { From intrinsic } \\
\text { experiences to } \\
\text { extrinsic } \\
\text { representations }\end{array}$ & Autonomy \\
\hline $\begin{array}{l}\text { "Do it for the Gram:" } \\
\text { "If you follow heaps of } \\
\text { celebs and stuff and } \\
\text { you see their bodies and } \\
\text { their lives and stuff, } \\
\text { you start to compare it } \\
\text { to yours, and you're } \\
\text { like 'oh mine's so } \\
\text { know. }\end{array}$ & Image & Effects of exposure & Competence \\
\hline
\end{tabular}

Using the social constructivist approach of critical emancipation, interviews with participants were analysed to discover patterns and themes in answer to my research questions. The following three chapters discuss the findings. 


\title{
6. The "social" in social media: Instagram and Relatedness
}

\author{
"Connection-isn't that why we all started going online in the first \\ place?” \\ -Aza Raskin (Center for Humane Technology, 2019)
}

\subsection{Introduction}

Social media, as is clear from the term itself, predominantly frames itself as a tool for social connection. In line with the literature on social media (Lee et al., 2015; Sheldon \& Bryant, 2016), participants consistently stated that connecting to others was their primary reason for using Instagram. As having close personal relationships is an intrinsic, self-transcendent value (Ryan \& Deci, 2017), this is an area where Instagram has a direct capacity, and expectation, to fulfil needs concerning social connection.

This chapter discusses participants' experiences of connection on Instagram in relation to the basic human need of relatedness. SDT posits that "feeling relatedness with others is an intrinsic and basic psychological need-something proximately valued for its own sake. Because close relationships have so consistently yielded significant adaptive benefits to individuals, we have evolved to be intrinsically motivated to seek out and maintain close, open, trusting relationships with others" (Ryan \& Deci, 2017, p. 294). Relatedness is important not only for associated benefits such as access to resources for survival, but in its own right: relationships are necessary for human development and wellbeing.

SDT proposes that interpersonal relationships can "not only fail to satisfy a person's basic needs, but also may actively thwart them" (2017, p. 305). When a relationship supports a person's feeling of autonomy, they will be more able to "be themselves- 
that is, to be authentic and transparent and to function closer to their own ideals" (2017, p. 308). Conversely, interactions can also be need-thwarting if they "convey a disconnection or absence of relatedness and thus undermine energy and wellness" (2017, p. 296). If one's feeling of relatedness is thwarted, they are likely to search for need satisfaction through extrinsic/materialistic mechanisms. As discussed throughout this thesis, thwarted basic human needs, and consequent extrinsic motivations, are antithetical to intrinsic values and thereby to environmental values. Consequently, the capacity for Instagram to support or thwart human connection can have a significant impact on values, and therefore, considering the structure of values (see Sections 2.2 and 2.4), environmentally responsible behaviour.

Within this chapter, I first examine how Instagram provided participants with a sense of community and reaffirmed aspects of identity, providing networks of support which may not be available to them in their offline lives. I then discuss memes as a modern form of keeping in contact and maintaining relationships. Following this, I explore participants' experiences of differences between online and offline connection, with examples demonstrating how online connection may not satisfy needs for relatedness. Finally, I look at how Instagram may be impacting offline connection. Instagram can facilitate friendships as a shared hobby, while it can also diminish in-person interactions through a preoccupation with posting to, or browsing, Instagram, to the detriment of the interactions themselves.

\subsection{Acceptance and identity in online communities}

Comments from participants demonstrated situations in which Instagram can offer users feelings of acceptance and community. For example, Georgia described how her brother was shy and uncomfortable in offline situations, but had found acceptance and connection online. Billie described a similar personal experience, finding that when her offline life was not fulfilling basic needs, Instagram gave her an alternative outlet to express herself, which helped her grow in confidence and build her offline identity as an 'influencer:' 
"I met so many people through Instagram, and I've been to events through Instagram where I've met new people and it's helped me socialise and get out of my comfort zone." -Billie

Billie described herself as shy and struggling to 'fit in' during her early teenage years. She found that Instagram provided a more comfortable outlet for selfexpression and identity exploration. For individuals whose basic needs are thwarted in offline environments, social media may provide alternative opportunities for relatedness. For instance, Facebook has been demonstrated to increase wellbeing for people high in anxiety, as an "alternative social outlet for developing and maintaining relationships" (Conger \& Singg, 2020, p. 41). For individuals who feel restricted or uncomfortable in offline spaces, online communities can provide a safer space for self-expression and relationship-building with like-minded individuals (RSPH, 2017).

For Billie, growing her identity as an Instagrammer translated to more social confidence and opportunities for relatedness in her offline life. SDT states that identities form at least partly through a desire to achieve relatedness. One may internalise the values of a social group in order to gain a sense of connection to that group, and "find belongingness within the social order" (Ryan \& Deci, 2017, p. 386). Billie embraced the identity of the Instagram influencer, aspiring to the lifestyles and values she saw modelling success on the platform. Through gaining confidence in this identity, and the social opportunities it offered, Billie became more confident and comfortable expressing herself offline, indicating some contribution from Instagram to broader feelings of relatedness.

Of note, this community and identity was available to Billie as a conventionally attractive young white female, and thereby fitting existing ideals of an Instagram influencer. A multitude of research explores how Instagram supports or subverts traditional media representations. Social media platforms remain "mediated by the same inequalities shaping real life" (Edwards \& Esposito, 2018, p. 342), with unequal access to success on the platform (Newlands \& Fieseler, 2020). A range of comments from participants demonstrated their own experiences exploring and building identity within Instagram. Participants in Group Interview 
One (GI1) discussed how Instagram aided in solidifying their identities as environmental activists, exemplified below by Jen:

"In a way it does make you want to stick to your grounding and your actual beliefs, and make you want to, I don't know, in a way I guess commit to this lifestyle." -Jen.

For GI1, a useful aspect of Instagram is that it provides public space for the expression of values and beliefs, which are supported and validated by like-minded online communities. As an outlet for creative expression, Instagram provides infrastructural space for users to explore identity; a platform to share thoughts and feelings and expressions of self. Building identity within a public social space may encourage aspects of identity which are endorsed in these online communities. Online communities can offer access to support networks that may not be accessible offline, allowing geographically disparate yet like-minded people to connect and share experiences.
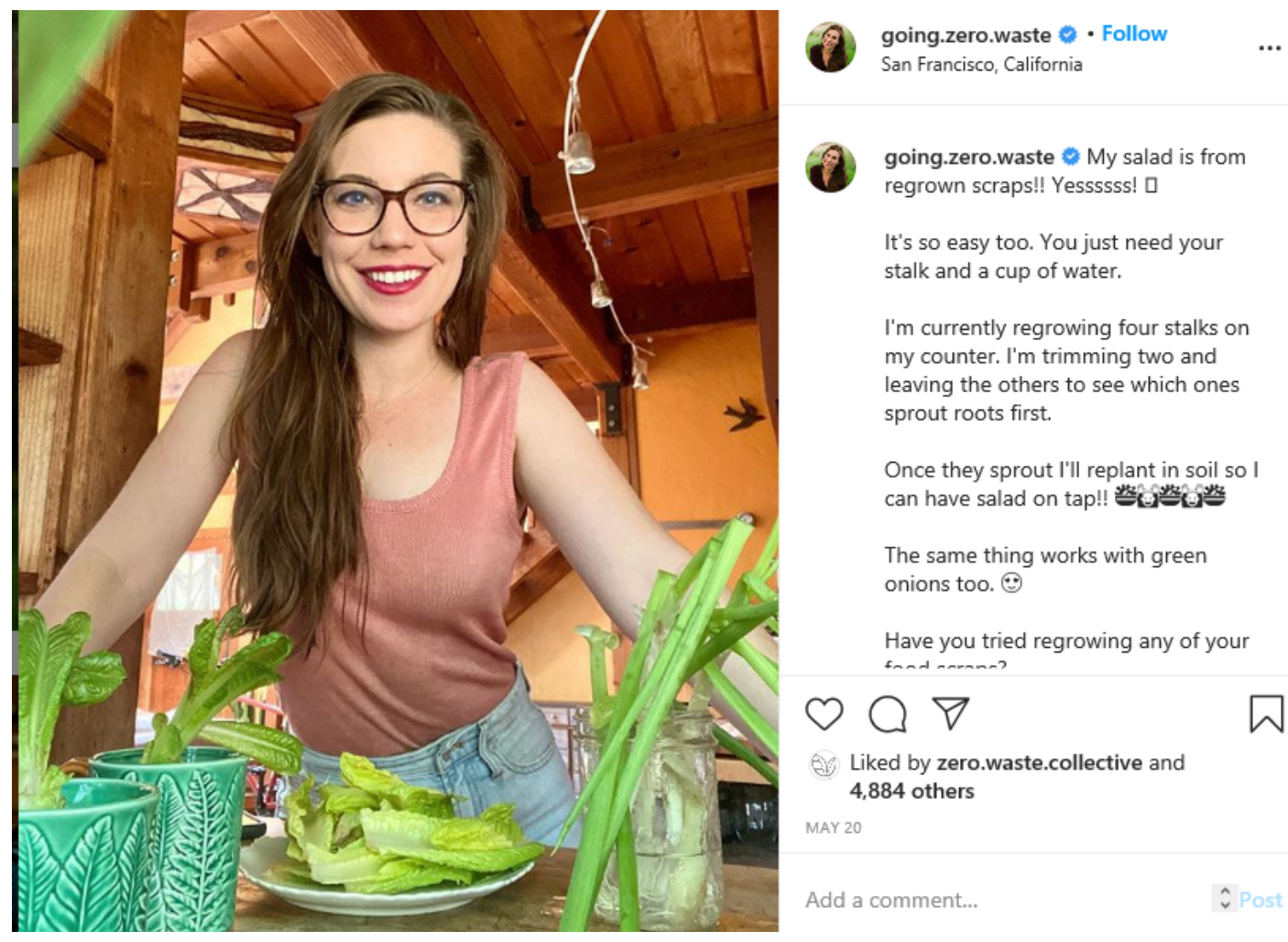

Example of an Instagram post promoting identity and community around "zero waste." Source: going_zero_waste https://www.instagram.com/p/CAYZJ4CH-fC/ 
Several participants recounted experiencing feelings of solidarity and support on Instagram. Group Interview Two (GI2) discussed social media in which female-only groups can ask and offer advice, often on very personal topics:

"A lot of people post stuff on there, and ask for comments- and this is like really, you know, intense stuff. Like 'I was sexually assaulted, and has anyone had this experience, what should I do?' Because it's a group of just females. I think there's quite a few people on it, maybe like definitely in the tens of thousands, but everyone's really accommodating, and is like 'I had this experience, private message me, ' and I feel like no one knows each other but-because it's such as large group of people-but I still feel like, if I had an experience and I really just needed a wider group of people to talk to, I would post on there." -Emily

This online community offers a support network in which, though no one knows each other at a personal level, the group environment allows members to feel comfortable enough to ask for help on profoundly personal issues. This environment appears to contribute to feelings of relatedness, where, as defined by SDT, one feels supported and that they reciprocally support others. Georgia also spoke of experiences of Instagram acting as a framework for like-minded individuals to connect over particular issues:

"There's quite a lot of love and support on this platform too, I get loads of messages from people who I don't even know, who are like 'hey I follow your work and just want to say you've personally inspired me'...I know a lot of people who definitely use the platform as a way of connecting over certain issues, and like finding kind of that support, and it's weird because they've developed relationships with people that they don't even know." Georgia

In these examples from Emily and Georgia, Instagram facilitates connections for group identities, whether sharing advice and support between women, or for connecting over a particular resonant issue. Users may, therefore, feel an extended circle of support, particularly if they do not receive that support in their offline life. While specific personal relationships are a more traditional source of support and solidarity, online communities such as Instagram instead provide support through a continually evolving network. As described by Blommaert and Varis, online 
communities "do not require the kinds of strong and lasting bonds grounded in shared bodies of knowledge we associate with more traditionally conceived "communities" or "societies" " (2014, p. 14). Platforms such as Instagram allow much broader access to other people, unlimited by geography. The vastness of these online communities offers users access to connection through a virtual agglomerate, where people can support or feel supported without "knowing" anyone in a traditional, offline capacity. Through these vast online communities, Instagram can support or enhance relatedness, thereby contributing to basic human needs.

\subsection{Connection through memes}

Memes, defined by Blommaert and Varis as "multimodal signs in which images and texts are combined" (2014, p. 8), are widespread on social media. People can 'tag" each other in memes, posting someone's name in the comments of a post (preceded by a '@') so they get a notification to view the post, alongside seeing who tagged them in it, and perhaps further comments or explanation. These tags are visible to anyone in the group or following the page in which the meme was posted.

Group Interview Two (GI2) discussed maintaining a connection to friends and family through social media memes, referencing memes as a way to understand the closeness of a relationship. For example:

\section{"You wouldn't tag someone that you weren't close with on some level" \\ -Dan \\ "I was having a fight with one of my friends, and we stopped tagging each other in memes, and it was like 'that's serious'" - Lisa}

Both of these comments indicate how individuals may use memes as a mechanism to understand the quality of a relationship. Dan's comment exemplifies how being tagged in a meme indicates to him a level of closeness. Conversely, Lisa understands the severity of her argument with her friend when they stop tagging each other in memes. Emily also spoke of memes as being her primary form of communication with her brother: 
"We live in the same city and I barely see him, but we tag each other in memes and I still feel close to him." -Emily

This example shows the ease and accessibility of communicating through memes. Although Emily rarely sees her brother, she nevertheless feels a close sense of connection due to regular communication through memes. Though there is no capacity for in-depth or complex discussion, being tagged reaffirms shared interest or humour, and is a signal that one is being thought of and is therefore important to the 'tagger.' Seft's 1995 study demonstrates the importance of "utterances that are said to have exclusively social, bonding functions like establishing and maintaining a friendly and harmonious atmosphere in interpersonal relations, especially during the opening and closing stages of social - verbal - encounters. These utterances are understood as a means for keeping the communication channels open" (as quoted in Blommeart \& Varis, 2014, p. 2). Tagging in Instagram memes may be an example of bonding functions which seem inconsequential but play an important role in maintaining a relationship. Further, memes often centre around humour, which as noted by Kuipers is "one of the strongest markers of social solidarity" and is "strongly connected with emotional closeness, solidarity and trust" (2009, p. 1). Memes provide appeal from being part of an 'in joke' which facilitates feelings of 'in-group-ness' (Miltner, 2014). 


\section{me trying to do a sit-up}

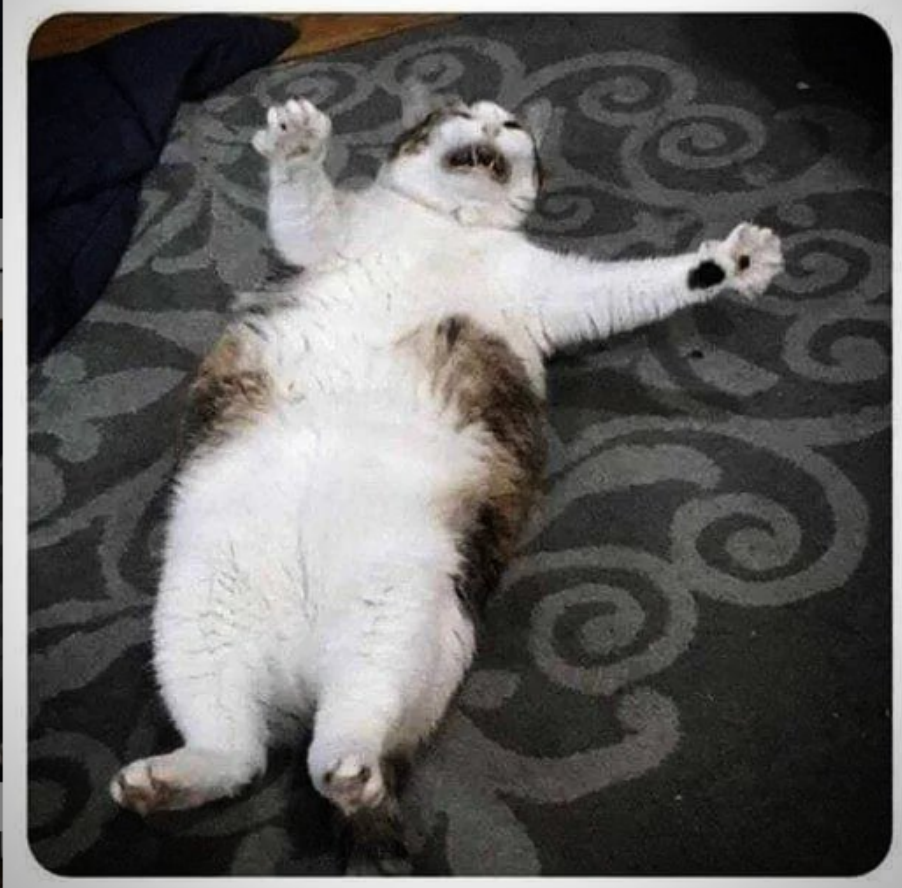

pets_have_humor - Follow

pets_have_humor Tag your "fitness" buddy 8

Follow at @pets_have_humor Follow at @pets_have_humor

\#catsofinstagram \#dogsofinstagram \#petsofinstagram \#goofy \#pets \#petmemes \#catmemes \#dogmemes \#cowsofinstagram \#funnymemes \#dankmemes \#animalmemes \#memes \#daily \#pussinboots \#adorable \#kittensofinstagram \#puppiesofinstagram \#pets \#petsofinstagram \#fitnessgoals \#fitness \#gymmotivation \#gym

\section{$0 \bigcirc \nabla$}

9. Liked by essie.wessie and 227 others AUGUST 23

Example of a meme on Instagram, including instruction to 'tag' friends. Source:

@pets_have_humor https://www.instagram.com/pets_have_humor/

The public nature of memes means that mutual friends are exposed to the tags, even when not purposely included in them, which can have varied outcomes. Generally, in both group interview discussions, participants grew noticeably animated when a familiar meme, group or social media trend was mentioned. In GI1, participants shared experiences of past social media trends, and even though they did not know each other at the time of specified trends, the shared cultural reference appeared to aid group connection. In GI2, Lisa clearly enjoyed being "in on" seeing the tags between Emily and her brother. However, she also stated that in a situation where she sees someone else tagged and feels she herself is "deserving of being tagged," she would feel excluded, a sentiment echoed by Billie. With the facilitation of "ingroup-ness" mentioned above, there inherently also comes a risk of exclusion from the in-group. People may feel excluded from specific tags, or, as discussed by Miltner (2014), entire social media groups or pages can be exclusionary or oppressive to some identities. While I wish to acknowledge that systematic exclusion exists among online communities and will have significant impacts on providing 
need-supporting or need-thwarting environments, a fuller discussion on this topic is beyond the scope of this thesis.

Discussions with participants demonstrate that tagging and being tagged in memes was overall conducive to supporting their feelings of relatedness. As a form of interaction, it reaffirmed shared interest or humour, enabled regular, easily accessible contact, and provided indication of a close and healthy relationship.

\subsection{Unsatisfying online connection and the need for external gratification}

Despite the above examples of achieving connection through Instagram, participants indicated other ways Instagram interactions might not be sufficient in fulfilling a sense of relatedness. Several participants mentioned limitations in building online relationships, with the format inherently reducing users to a simplified avatar of themselves. With communication limited to images and written text, it can be challenging to convey nuance, or to 'read' emotions and respond appropriately, as discussed by Georgia:

\footnotetext{
"Sometimes I've been like, is it good to connect all these people and then not have any appreciation of them as very complex multifaceted human beings, like which you would if you were able to engage with them face to face? Especially at times when we've had conflict within the (climate) movement I'm like, this would be so much easier to resolve if people were able to have an appreciation of the whole person not just their little face on a group chat, which allows you to say all sorts of things you wouldn't say to someone in person." -Georgia
}

Georgia's comments concerning communicating online in ways that differ from face to face interactions are explained by the concept of 'online disinhibition' (Suler, 2005); a theory stating that due to the relative anonymity of online interactions, typical inhibitors to participating in harmful behaviour are removed. The effects of harmful behaviour are invisible to the perpetrator, distanced by time or geography. Further, the limited range of social cues available in online interactions means the perpetrator receives minimal feedback on the inappropriateness of their behaviour, such as they would otherwise in face-to-face communication (Casale \& Fioravanti, 
2018). Therefore, online disinhibition may incentivise or magnify harmful interactions, actively thwarting relatedness.

Other participants discussed experiencing online interaction as less satisfying than offline interaction. For instance, Lisa, when explaining why she temporarily deleted her Instagram, stated:

\section{"I think I just needed a break from people. Coz I feel like the interactions online are just different to the interactions in person. And I needed that refreshing in-person interaction that I wasn't getting online, if that makes sense. So, I mean it was still pretty pathetic to not go on for like 24 hours but that did actually feel like a pretty decent break for me." -Lisa}

Lisa simultaneously states a need for both "a break from people" and more "inperson interaction," indicating that despite having an overload of online connection, it did not satisfy her need for relatedness. Despite this perception, her 'break' was only 24 hours, indicating that although she realised Instagram's limitations in contributing to relatedness, she still felt strong incentives to use it daily. This experience demonstrates the addictive nature of Instagram: Research has demonstrated Instagram to be more addictive than cigarettes and alcohol (RSPH, 2017). By repeatedly drawing in the attention of users, this takes time and attention away from potentially more fulfilling offline interactions. Lisa's experience demonstrates that, while there is significant access to connection on Instagram, it is not necessarily quality connection and may not satisfy users' needs for relatedness.

Amy also experienced differences between online and offline interactions. She discussed how she relies on communication in the form of likes and comments on her photos to gain confidence in the way she looks. When asked if she would find it difficult to delete Instagram and no longer have these interactions, she stated:

"I think at first it would be, but also it might make it more valuable, like face to face interaction when someone says something nice, almost. At first, I think it would be hard and weird, but I think you'd just get used to it and that's way better, in my opinion." - Amy 
Like Lisa, Amy believes that offline interaction and support would be preferable to the online interactions she has. Perhaps due to the image-based and self-promotional nature of Instagram, interactions can more readily centre around giving and receiving image-based gratification. A reliance on others to give support in the form of imagebased gratification was a frequent topic of discussion among participants. For instance, when asked why she feels the need to post her photos on Instagram, Amy replied:
"I don't know, I think it's almost like...reassurance from people that you do look nice, and you do look good, you need that ego boost. Or like if you think you look nice then you've gotta make sure other people see that side of you and...almost like that. When people comment on it and stuff and you're like 'ok yeah, I do look OK, that's good. If people like it then I do actually look good.' " - Amy

Amy's experience is an example of SDT's concept of 'contingent self-esteem' (Ryan \& Deci, 2017), in which feelings of self-worth are dependent on validation from others. Interactions focussing on gratification concerning one's appearance further incentivises image as an important value, as it is reinforced through peer communication. Further, success in appearance is 'controlled' in that it is measured in approval from others, and therefore thwarting the basic human need of autonomy. Individuals feel a perceived pressure to "prove one's worth to others" (2017, p. 168), and rely on compliments from others to earn or maintain self-worth.

While Amy spoke of posting images to validate her positive self-image, participants also spoke of posting for reassurance when they are feeling low. When asked why she needed online validation, Billie replied:

\section{"I don't know, sometimes you get lonely. So sometimes it's nice to like- when you're having a shit day just post a photo of yourself and then people are like 'oh you're so pretty, 'whatever. It just makes you feel good I guess." -Billie}

Billie's experience aligns with a Singaporean qualitative study of girls using Instagram, which found that their participants made efforts to present attractive images of themselves on social media in order to gain a sense of self-worth (Chua \& 
Chang, 2016). The study theorizes that for users feeling low in self-esteem, Instagram allows them to carefully curate and edit images of themselves at their best, aiming to improve self-esteem through receiving image-based gratification from others. Gaining self-worth and feelings of relatedness through image-based gratification is likely to act as "substitutes or symbolic satisfiers of more basic needs that have been thwarted" (Ryan \& Deci, 2017, p. 91). As applied to Billie's experience above, feelings of loneliness are an example of a thwarted need for relatedness, which she attempts to rectify through the more temporary, symbolic satisfier of online image-based gratification. However, while this may temporarily improve mood, this self-worth is conditional on a constructed appearance, and, being outside Billie's control, needs consistent reaffirmation to avoid returning to previous lower levels of self-esteem. The perpetual pursuit of reaffirmation maintains attention on image and the pursuit of gratification, 'crowding out' intrinsic aspirations that would better fulfil basic needs (Kasser, 2016).

A further issue with connection centring on online gratification is that it is inherently a comparatively limited view of a person's actual lived experience. The impetus for gratification, alongside the tendency for Instagram to show the "shiny" parts of life, may lead to uncontextualised images, which can lead to interactions with unintended negative consequences, For example, Laura found uncontextualized interactions exacerbated her eating disorder:

\section{"And also the instant gratification, you know, you post a photo and then people are like 'oh you look so good' and it's like, 'thanks, I haven't eaten in three days!'” -Laura}

While those commenting on Laura's photo may be well-meaning in their compliments, the separation from context makes it difficult to have any sense of the person's experience outside the constructed image. As evident from Laura's statement above, responses intended as supportive comments can inadvertently be an incentive to exacerbate dangerously unhealthy habits. An individual's goal to lose weight, even at an unhealthy rate, can be experienced as successful if others comment positively on their image (Ryan \& Deci, 2017). These measures of success can thus be self-reinforcing, perpetuating unhealthy eating habits. This outcome is 
evident in the above example from Laura, who experienced gratification from her peers, reinforcing harmful behaviours.

\subsection{Instagram and offline connection}

Further to discussing interactions within the Instagram infrastructure, themes emerged related to how Instagram impacts offline connection. Participants stated a multitude of experiences where Instagram influenced offline connection, with a variety of outcomes. Like any shared interest, a passion for Instagram can aid relationship-building with other, like-minded Instagram users, as described by Billie and Amy:

"I really bonded with this girl overseas coz she was super into Instagram and so was I, and we would just kind of run away from the group and go on hikes and like-to take photos-but it was like fun, but we were doing it to take photos. We did have a good time and we enjoyed it." -Billie

"I can think of really fun times off the top of my head where like me and my friend have gone somewhere really beautiful and we've spent ages taking photos and doing fun stuff, I definitely think it can add to the fun." -Amy

These experiences are not about the quality of connection on Instagram itself, but rather how their shared interest in Instagram facilitates offline connection. In the above examples, Instagram aided in both forging a friendship and maintaining one; thus, contributing to feelings of relatedness.

However, others described experiences in which Instagram was detrimental to offline relationships. They felt diminished in-person connection due to friends' preoccupation with Instagram while spending time together. This emerged in two ways: Firstly, participants experienced frustration when friends were predominantly focussed on representing their offline experiences on Instagram, rather than the interaction itself:

"I definitely know friends who are posting every aspect of their lives on (Instagram), and its- I personally find it difficult to be around, or like, we'll be hanging out, and it's just about like "oh let's take a photo of this' or whatever, rather than -which to me 
feels like detracting from the current moment, if you're constantly feeling the need to project it to some people who aren't even there, like, just enjoy the people that are." -Georgia

Georgia's comment, a view also shared by other participants, shows a frustration that time spent together is hindered when friends are absorbed by Instagram rather than being fully present. For Georgia, the quality of the interaction is impacted by her friends' desire to promote it to others. The offline interaction thus becomes an avenue for gaining status on Instagram, as opposed to being enjoyed for its own sake. This alludes to the capacity for the intrinsic experience of enjoying quality time with friends to perhaps become more focussed on the extrinsic desire to portray that experience to others as a means of self-promotion; thereby reducing the quality of the interaction itself.

Secondly, participants also spoke of impacts on offline interactions arising from spending time with people who were simultaneously scrolling through Instagram. For instance, Laura did not realise how distracted her offline interactions were until she had a period of time away from all social media:

"When I was on my social media detox I'd wanna chat and they'd
just be on their phones, like talking to me but like, scrolling at the
same time. And I was like 'woah, I can't believe this is what it's
like.' So ever since then I've tried to make a conscious effort if I'm
talking to someone, I'll put my phone away and not look at it." -
Laura

This quote from Laura exemplifies the difficulty of offline connection when Instagram is simultaneously taking attention away from the interaction. A preoccupation with Instagram limits offline connection not only for the person on their phone, but also for those around them who find themselves competing with Instagram for their friends' attention. Laura was not aware of the extent to which Instagram drew attention away from her offline interactions until she no longer had her phone.

Laura's experience is not unusual: A small but growing body of research examines the effects of what has been termed "phubbing," when a person is preoccupied with 
their smartphone and thereby ignores physically present company. One study found that $89 \%$ of participants had used their smartphone during their most recent social activity (Rainie \& Zickuhr, 2015). The term 'phubbing' describes a form of social exclusion which thwarts feelings of belongingness (Chotpitayasunondh \& Douglas, 2018). A recent study found that nearly half of participants had been 'phubbed' by a romantic partner (Roberts \& David, 2016). Through both posting about interactions on Instagram, and phubbing by looking at others' posts on Instagram, the platform can hinder offline connection, thereby thwarting relatedness.

\subsection{Conclusion}

Participant interviews confirmed a key motivation for their use of Instagram is to forge and maintain connections to others. According to SDT, interactions can either support or thwart relatedness, the feeling of supporting and being supported by others, which is one of the three universal human needs. When these human needs are met, intrinsic values are supported. When they are thwarted, individuals often uptake extrinsic values as substitutes, which has correlations with lower levels of mental well-being and less environmentally responsible behaviour.

Participants discussed instances of gaining connection, support and solidarity on Instagram, particularly when they may not have access to, or feel comfortable in, such networks in offline life. Participants shared experiences of maintaining communication through the platform, or, further, forming or maintaining offline friendships through a shared interest in Instagram.

However, further participant experiences indicate ways Instagram is not supporting, or is actively thwarting, their feelings of relatedness. Participants perceived a difference in the quality of Instagram versus offline interactions, stating examples in which, despite virtually infinite access to other people through Instagram, this access did not satisfy their need for relatedness. SDT states that when basic human needs are unfulfilled, there is a tendency to attempt to gain a semblance of these fulfilments through extrinsically oriented substitutes. Therein, Instagram users who feel a thwarted sense of relatedness are more likely to adopt extrinsic aspirations to find relatedness through alternative means, for instance, through receiving image-based online gratification. However, such gratification will not fulfil relatedness either, as 
it exacerbates using interactions as a means to an end (searching for approval from others) as opposed to the intrinsic enjoyment of the relationship. These examples indicate ways in which Instagram may be a need-thwarting environment, thus contributing to extrinsic values.

Participants also voiced frustrations that friends were often too preoccupied with posting to Instagram, or viewing images from others, to be fully 'present' in offline interactions. This indicates the intrinsic experience of offline connection being subsumed by the extrinsic experience of representing interactions to others in pursuit of social approval. 


\title{
7. Social comparison, thwarted competence and materialism
}

\author{
"Just like scrolling seeing all these girls with just perfect everything is- I \\ feel like that affects you in a negative way"
}

- Billie, GI2

\subsection{Introduction}

'Social comparison theory' entered academic literature in the mid- $20^{\text {th }}$ century, describing an instinct to compare oneself to others; both in order to learn and grow, and to be reassured that one's own opinions and abilities are in line with others' (Festinger, 1954). However, more recent research shows social comparison can lead to adverse outcomes. For instance, excessive 'upward' social comparison (comparing oneself to others seen as more successful) can lead to feelings of incompetence and low self-worth. These feelings of incompetence predict stronger materialistic aspirations, as individuals attempt to regain self-worth through extrinsic pursuits (Kasser, 2016; Richins, 2017). As stated by Kasser, all people have the capacity to behave materialistically, and therefore researchers must ask, rather than who is materialistic, "when are people materialistic?" (2016, p. 506). This chapter thus explores how thwarted competence through social comparison leads to increased materialistic values.

One of the most commonly arising themes in interviews was the tendency for participants to compare themselves to the people they saw on Instagram. Instagram tends to show an idealized version of one's life, a sentiment voiced by many participants and established in research (de Vries \& Kühne, 2015; Ho \& Ito, 2019; Sherlock \& Wagstaff, 2019). Participants frequently spoke of viewing carefully curated 'highlight reels' on Instagram and feeling that themselves and their own lives were inadequate in comparison. They perceived a certain standard of success, beauty, or excitement portrayed on Instagram, which they often felt unable to reach personally. 
In this chapter, I explore participants' experiences of social comparison on Instagram in relation to the basic human need of competence. Firstly, I will discuss participants' experiences of feeling relatively inadequate on Instagram, indicating thwarted feelings of competence. I then examine participants' experiences through ego/task orientated social comparison. Following this, I discuss the variation in effects when participants compare themselves to friends versus celebrities, as predicted by Lup (2015) and Hwnag (2019). Lastly, I explore the direct relationship between upward social comparison and materialism.

\subsection{Effects of exposure to idealised content}

Many participants spoke of feeling that their lives were inferior to the lives they saw portrayed on Instagram, even while aware that the online representations depicted, for example, "highlight reels" of life (Georgia), only the "positive, happy things" (Amy), or a "fake reality" (Billie). Participants mentioned a range of interests they followed in which seeing the images left them feeling inadequate in comparison. For instance, they viewed Instagram feeds showing people travelling while they were not, people more productive in terms of activism than they were, living a more exciting life than they were, or attaining higher levels of fitness. Each of these participants mentioned feeling adverse psychological outcomes from seeing this content, despite consciously knowing it was not representative of anyone's 'reality.' Seeing screeds of images of people appearing successful in various ways, participants felt unable to keep up with a perceived standard. Vanderckhove (2019) discusses how perceiving an unrealistically high standard, and failing to reach it, can lead to feelings of self-criticism. Instagram's idealised depiction of life creates a standard which is highly edited and therefore artificially raised, increasing users' expectations of what they should be achieving for themselves.

Viewing idealized images on Instagram leads users to experience frustrated feelings of competence when they inevitably cannot reach the perceived standard in their own real-life experience. As competence is one of the basic human needs, when it is thwarted, this directly affects levels of wellbeing. As discussed in Section 2.4, individuals with thwarted basic human needs are more likely to seek out extrinsic goals. According to Vanderkerckhove et al, "persistent need frustration leads to 
feelings of insecurity and this makes individuals prone to the pursuit of extrinsic goals" (2019, p. 584). Experiencing frequently frustrated feelings of competence, individuals attempt to regain competence through indirect or symbolic means, which often involves purchasing a product which is advertised as a way to feel better about oneself (Kasser \& Kanner, 2004).

Many participants particularly stated feeling more severe negative psychological impacts from Instagram use when they were younger. For instance, Laura discussed a lessening of perceived pressure to create successful Instagram content:

\section{"I just don't really care anymore. It could be an age thing, it could also be I've just fought my battle, like social media and my self-esteem and my self-worth with that" -Laura}

While many participants took a philosophical view of having more difficult struggles with Instagram and self-esteem when they were younger, it nevertheless remains concerning that the struggle exists at all. Furthermore, in recent years, since these participants have largely 'fought their battles,' the platforms have only become both more popular and more commercialised (Statista, 2020). With adolescents the primary demographic of Instagram users (Sherlock \& Wagstaff, 2019), as well as the most susceptible demographic to self-criticism (Ryan \& Deci, 2017), greater care and protection needs to be put in place to protect adolescents, in particular, from the worst effects of the Instagram social context.

\subsection{Social comparison and task/ego orientation}

As stated above, a consistent theme amongst almost all participants was a feeling of pressure to reach a certain standard in their own lives which they perceived as the norm on Instagram. Participants stated feeling unable to achieve a perceived standard of beauty, or that their life was less exciting, productive or high achieving than what they saw on Instagram. Participants frequently experienced feelings of inadequacy when viewing, and concurrently comparing themselves to, images they saw on Instagram.

Social comparison theory (Festinger, 1954) states that people have an innate desire to compare themselves against others as a way to evaluate their own opinions and 
abilities. Social comparison manifests in 3 forms (Hwnag, 2019): Upward social comparison occurs when people compare themselves with others whom they perceive as more successful and tends to be motivated by a desire for selfimprovement. However, it can also lead to adverse mental health outcomes by making individuals feel personally inadequate. Downward social comparison occurs when people compare themselves with others who are less successful than themselves and tends to be motivated by a search for self-reassurance. Lastly, the most frequently employed form of social comparison is horizontal, which functions as a way for individuals to check that their opinions and abilities are in line with others.

SDT describes the differentiation between task-orientation, an intrinsic desire to grow and learn, and ego-orientation, an extrinsic desire to prove one's worth to oneself or others (Ryan \& Deci, 2017). Butler (1993) merged the theories of socialcomparison and task/ego orientation. She discusses how social comparison differs according to two possible motivating factors, with accordingly different outcomes: When individuals compare themselves to others of greater ability in order to learn and grow it is 'task-oriented' social comparison. On the other hand, when an individual measures themselves against others in order to reassure them of their own abilities and maintain self-worth, it is 'ego-oriented' social comparison.

Billie spoke of following people on Instagram because they inspire her; signifying task-oriented social comparison's desire to learn and grow:

\section{"I guess it's kind of an aspiration of like, 'oh I wish I could do that,' that kind of thing. Or that's like what I've seen through Instagram and who my friends follow and who I follow. Like I don't follow someone who- that's boring online I guess... I just wanna see exciting things that I can-I guess- not aspire to be but, I don't know... I guess if you see people taking nice photos at nice restaurants you're like 'ooh I wanna do that.' Or like nice travel photos it's like 'ooh I wanna go there.' "-Billie}

While Billie's experience appears task-oriented, she found that following 'inspiring' profiles, predominantly image-focussed celebrities and influencers, often left her feeling psychologically worse: 
"I feel like people follow people because they want to be inspired, but then it kind of flips and they're like, 'oh I don't have that, I'm not good enough."-Billie

Billie's comment demonstrates a blend of both task (inspiration) and ego (evaluating selfworth) orientation within one context. This convergence is not addressed by Butler, who measured motivation as being either one way or the other. However, Billie's experience indicates a more fluid relationship between social comparison orientations. For instance, Billie has a desire to learn and grow, looking at images on Instagram for inspiration. She thus enters the social comparison with a task-oriented motivation. When viewing the images, due to the 'highlight reel' nature of Instagram, she predominantly employs upward social comparison, seeing polished images of exciting lives. Assuming both task-oriented and egooriented social comparison serve an important function to psychological wellbeing, a problem arises: As Instagram tends to show an idealised version of reality, it creates an unreasonably high 'standard,' as users compare the 'highlight reel' versions of life they see on Instagram with their own realities.

When users employ ego-oriented social comparison to assess their abilities are in line with others, the content to measure themselves against is unrealistic and idealized. Users thereby employ an excess of upward social comparison, comparing themselves with these artificially raised standards. There is a lack of opportunity for horizontal or downward social comparison, which would serve the purpose of reassuring users that their opinions and abilities are in line with others. This imbalance may result in individuals searching for such self-evaluation through upward social comparison instead, thereby leading to feelings of incompetence or inadequacy, as discussed by Billie above.

The nature of the images Billie views on Instagram further complicate her initial task-involved motivation. The predominant images she sees celebrate the extrinsic values of wealth, image and status (see Section 6.4). Billie's task-oriented motivation to improve herself is channelled to extrinsic aspirations, modelled on the values of the role models she aspires to be like. Success in these values is, however, inherently reliant on external measures such as approval from others (Ryan \& Deci, 2017). Billie is unable to define her own success in the qualitatively evaluated Instagram environment. This involves a thwarting of autonomy, another basic human need, and therefore likely to be detrimental to wellbeing. 
Thus, a more nuanced blend of task orientation and ego orientation emerges, with a desire to learn and grow, yet toward extrinsic goals that thwart basic human needs. As discussed throughout this research, a shift toward extrinsic orientation is a shift away from both mental and ecological wellbeing.

\subsection{Social comparison with friends vs celebrities}

Several participants discussed a perceived difference in how they were affected by viewing images of their friends versus images of celebrities or influencers. They consistently spoke of an increased negative effect when viewing more celebrity/influencer images, with the key difference evidently being that they knew more of their friends' real-life experiences and therefore had more awareness that their reality was less 'perfect' than it appeared on Instagram. The following quote from Georgia exemplifies this:

\footnotetext{
"I remember actually someone was talking about 'oh, I had to delete Instagram, it's just so difficult to see all these beautiful people and their very shiny lives, not to constantly compare myself against that and stuff,' and I was like 'oh...right,' because I just follow people that I know, so I know their lives aren't shiny. I mean obviously with your friends- it's definitely still projecting a false image of their lives, so sometimes it can be hard as well when you follow people that you know coz you're like 'oh wow these people are doing all this stuff that I should be too." -Georgia.
}

While Georgia still feels pressure resulting from comparing herself with her peers on Instagram, she is simultaneously aware of their broader context and less "shiny" parts of their lives. On the other hand, following celebrities or strangers on Instagram coveys only the constructed, idealised versions of themselves; separated from reallife context.

Further participants also spoke of feeling adverse psychological effects from following celebrity accounts, despite conscious awareness of the highly edited and constructed nature of these accounts. For instance, Laura discussed the significant adverse psychological effects she felt from comparing herself with celebrities: 


\section{"I think there's a lot of pressure to kind of push out a certain image of yourself on (Instagram). Now I don't follow any celebrities, I just follow people I know and food. Just coz I felt like the more verified accounts I followed, the more shit I felt about myself. I was just kind of comparing myself to all these people who just had a very different way of life, and it just made me feel bad about myself- I got an eating disorder from it." -Laura}

Laura stated that following celebrity Instagram accounts had a direct influence on her mental health and self-image. Her experiences, (similar to the accounts of other participants), of feeling more substantial adverse effects from following strangers as opposed to friends add qualitative validation to previous quantitative research. Studies from Lup et al. (2015) and Hwnag (2019) found that when social comparison was a mediator, higher depressive symptoms than average were correlated with following strangers on Instagram; while lower depressive symptoms than average were correlated with following friends. Hwnag proposed that Instagram's "prominence of positive images may trigger assumptions that these photos are indicative of how the people in them actually live and thus maybe people are more vulnerable to judging themselves in relation to the assumed (but often unrealistic) lives of others, which can trigger negative feelings (e.g. depression and stress)" (2019, p. 1630). When viewers know a person from outside the context of Instagram, they can more easily rationalise the images as being non-representative of that person's real-life experience. It is more challenging to do so for strangers' images, without any knowledge of their lives outside the polished context of Instagram.

While Lup (2015) and Hwang (2019) found an overall positive effect when individuals viewed friends' Instagram images, participants of this study stated negative experiences from friends as well; albeit to a smaller extent than from following celebrities. This departure from previous findings could have been due to a bias of what participants believed I wanted to hear, or perhaps the qualitative discussions allow a level of nuance not accounted for in the quantitative studies undertaken by Lup and Hwang. However, overall, the findings from Lup and Hwang that demonstrated a more pronounced negative psychological effect from following celebrities was maintained in the present thesis.

Participants experienced thwarted competence through social comparison with friends and, to a greater extent, celebrities on Instagram. SDT states that when basic 
human needs are unfulfilled, there is a tendency to attempt to gain a semblance of these fulfilments through other means. According to SDT, "Individuals whose basic psychological needs have been neglected or frustrated in development are more prone to adopt need substitutes, such as extrinsic life goals, as being personally important" (Ryan \& Deci, 2017, p. 285). As discussed in Section 2.1, research has shown that the most likely way to improve environmentally responsible behaviour is to disincentivise extrinsic values, and instead encourage more intrinsic values. A move toward the extrinsic side of the values circumplex is a move away from values associated with environmentally responsible behaviour.

\subsection{Social comparison and materialism}

As discussed above, Instagram's tendency to portray an idealised version of reality leads to a nimiety of upward social comparison, resulting in thwarted feelings of competence. Although participants were cognizant of Instagram's unrealistic 'standards,' participants frequently mentioned comparing themselves to the images they saw presented on the platform, and feeling inadequate as a result. For example, Billie stated:

\footnotetext{
"After coming off Instagram- you know you see all these pretty people with good bodies and good clothes, I would always just be like, 'oh I need to go shopping, I need new clothes, I need to get a nose job, I need to get a spray tan...' all of that bad stuff, and it's so easy for people on the internet to be like 'love yourself' but when you come off it it's kind of difficult, to deal with it yourself." -Billie
}

Exposure to images of conventionally attractive people on Instagram has led Billie to feel pressure to conform to a perceived standard of beauty. Visible themes stating to "love yourself" get lost among the other less explicit underlying messages primarily, that to be accepted, she must continuously strive to create and maintain an idealized self-representation that meets the perceived 'standard' depicted on Instagram. Keeping up with these perceived standards is unattainable, and therefore, likely to continually be a source of frustration. 


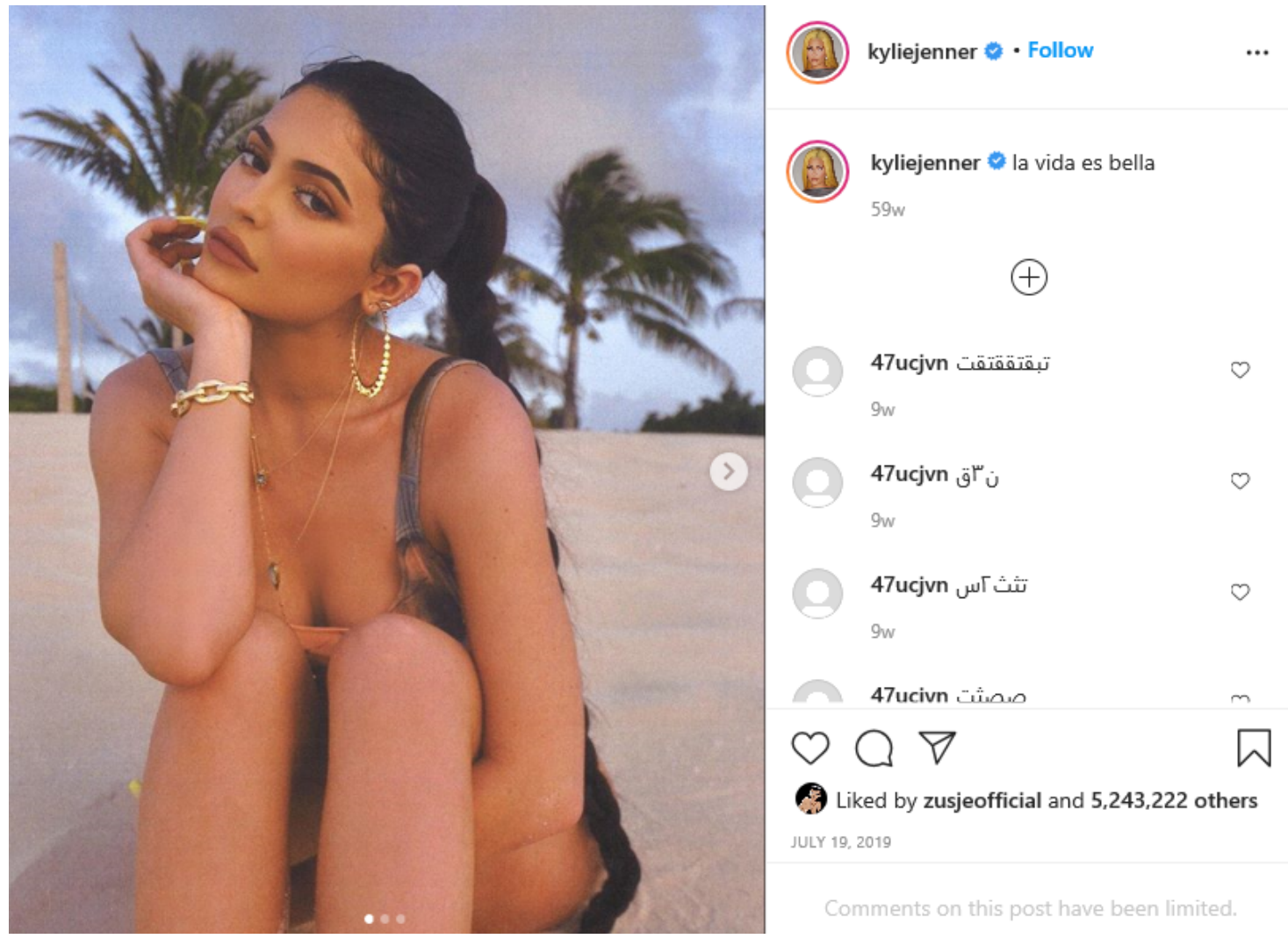

An example of a lifestyle depicted Instagram influencer. Source: @kyliejenner https://www.instagram.com/p/BOFOW6gndLo/

Richins argues that often individuals who feel negative psychological states tend to believe specific products have "transformative powers" which will increase their feelings of wellbeing (2017, p. 480). For instance, Billie sees images of attractive people and, feeling comparatively inadequate, desires products which she believes will enhance her appearance, bringing her up to the 'standard' she perceives. Purchasing the product, Richins continues, will cause individuals to feel briefly better, before returning to the same negative state, searching for another purchase to instil wellbeing, thus creating a perpetuating cycle of materialistic behaviour.

As a further consideration, Billie's expectations are artificially raised specifically in terms of self-presentation, centring the value of 'image.' This focus may be understood through previous research from De Vries \& Kühne (2015), who states that emerging adolescents tend to emphasise themselves as popular and attractive on social media. Exposure to images of others looking more popular or attractive than oneself is likely to inflict feelings of inadequacy in these areas, creating a desire to improve one's own image and status in order to reach the perceived standard. 
A similar process may be at work in terms of consumption practises. Repeated exposure to images showing consumptive behaviour can increase expectations around what is a 'normal' level of affluence (Richins, 2017). Laura stated that Instagram was a platform particularly suited to demonstrating messages of consumption:

"I think on Instagram especially you want to show off what you've got." -Laura

This quote from Laura demonstrates an immediate materialistic incentive through Instagram. Instagram offers greater access to conspicuous consumption, which involves "attaining and exhibiting costly items to impress upon others that one possesses wealth or status" (Sundie et al., 2011, p. 664), a definition which clearly shows the extrinsic motivations of the behaviour. Instagram offers users a potentially far greater 'audience' to impress with their purchases. Research indicates Laura's experience is not unusual: Krause et al (2019) found that frequency of Instagram use positively predicted engaging in conspicuous consumption. They reason that Instagram users see others participating in conspicuous consumption on the platform, and viewers 'approving' it, as demonstrated through likes and comments supporting the purchase. Krause et al. also found that higher levels of conspicuous consumption correlated with higher levels of envy toward other Instagram users, with evidence that conspicuous consumption was at least partly mediated by an "envy triggered behavioural response to close the gap between oneself and the envied other" (2019, p. 12). This echoes previously discussed themes of social comparison and materialism, in that users are exposed to ideals of affluence, feel like their own standard is 'below par,' and thus are incentivised to employ materialistic behaviours in efforts to repair feelings of competence.

\subsection{Conclusion}

Participants frequently engaged in social comparison when viewing Instagram images. As Instagram images tend to be highly curated, idealised depictions of life, participants particularly engaged in upward social comparison. While upward social comparison can be a source of inspiration and is important for self-development (Festinger, 1954), the overly idealized content on Instagram led to adverse outcomes 
for participants, with perceptions of personal inadequacy and thus thwarted competence.

Thwarted competence resulting from upward social comparison was stronger when participants viewed images of celebrities, in alignment with research from Hwnag (2019) and Lup (2015). However, in a departure from Huang and Lup, negative implications also existed, albeit to a smaller extent, when viewing images of friends. Some participants explicitly stated materialistic, image-related goals emerged from viewing images on Instagram. Thwarted competence is likely to increase extrinsic aspirations, such as materialistic tendencies, in an attempt to repair feelings of selfworth (Richins, 2017). Materialistic aspirations were further incentivised through participants' exposure to a plethora of idealised images depicting materialistic content (see Section 6.4). These images signal to users that the subjects in these images are more 'successful' than they are and incentivises them to strive to achieve similar outcomes for themselves.

In sum, through social comparison and thwarted competence, participants showed indication of being incentivised toward stronger materialistic values. Every user has a different experience of Instagram, and therefore, this analysis is far from conclusive. However, the experiences discussed by participants indicate the capacity for Instagram to exacerbate materialistic aspirations. If society is to overcome the current ecological crises and undertake the societal transformation requested by the IPCC (2018), there needs to be a shift in cultural values toward the intrinsic values associated with mental and ecological wellbeing. However, experiences of participants, in alignment with related research, indicates that the cultural values depicted on Instagram, and resultant outcomes through social comparison and SDT, are conducive to further exacerbating materialistic extrinsic values. Considering Instagram users number over a billion individuals, the misalignment between using Instagram and developing intrinsic cultural values may have deep-seated consequences in terms of responding to ecological crises. 


\title{
8. Autonomy: Incentives toward self-promotion
}

\author{
"As a medium, the internet is defined by a built-in performance \\ incentive. In real life, you can walk around living life and be visible to \\ other people. But you can't just walk around and be visible on the \\ internet-for anyone to see you, you have to act. You have to \\ communicate in order to maintain an internet presence. And, because the \\ internet's central platforms are built around personal profiles, it can \\ seem-first at a mechanical level, and later on as an encoded instinct-like \\ the main purpose of this communication is to make yourself look good" \\ (Tolentino, 2020, p. 8).
}

\subsection{Introduction}

The third basic human need, autonomy, describes feeling able to express oneself freely, without coercion. According to SDT, the opposite of autonomy is control; feeling pressure from external sources to present oneself a certain way (Ryan \& Deci, 2017). This is commonly experienced: "It is (perhaps too) frequently the case that people's behaviour arises not from the authentic strivings of the self but instead from feelings of coercion, control, and pressure (Deci \& Ryan, 2002, p. 127).” Autonomy and control closely interlink with intrinsic and extrinsic motivations. When one feels supported in their sense of autonomy, they are free to pursue their intrinsic interests. When, on the other hand, one experiences forms of control, they feel pressure to act according to the preferences of others, thus motivated extrinsically. Behaving a certain way according to even subtle or imagined preferences of others involves an internalised control, and therein, extrinsic motivation.

The premise of Instagram as a platform offering creative self-expression appears at first glance to support autonomy. However, participants spoke of experiencing pressure to present themselves in certain ways on Instagram. Several participants indicated that they did not feel free to express their genuine selves on Instagram, but instead felt compelled to post images according to what they believed their followers 
'wanted to see.' Users are cognizant that their images will be more 'successful' if they construct images in certain ways, such as constructing idealised images, centring the self, objectifying the self, and purposefully seeking out experiences for their photogenic appeal. These examples indicate ways the norms and infrastructure of Instagram act as controlling factors, influencing behaviour on the platform and thereby limiting autonomy.

In enacting these online behaviours, users are mimicking norms set by professional Instagram influencers, who are have large economic incentives to self-promote (Page, 2012). Influencers, as modern-day cultural messengers, shape the values of the platform, setting the tone as role models and ideals of 'success' which other users can aspire to. In this way Instagram, participating in a neoliberal economy and with its most celebrated figureheads espousing the values of wealth, image and fame, inherently endorses extrinsic values.

It should be noted that several participants stated that since Instagram stopped displaying how many 'likes' a post received (in July 2019 for users in New Zealand) the pressure to conform to Instagram norms had lessened. However, some pressures evidently remain- the vast majority of examples mentioned in this chapter were experienced after those changes were implemented.

\subsection{Like for like: Attention on Social Status}

Some participants spoke of participating in an Instagram trend to trade 'like for like' or 'follow for follow.' These phrases, prevalent on social media, proclaim that any likes and follows will be reciprocated, thus allowing both users to grow in popularity. In this situation, users 'like' or 'follow' a social media profile not because of a genuine interest in the content itself, but because they hope the action will be reciprocated. The 'like' or 'follow' is a means to an end, motivated by an outside factor- enhancing one's status -and thus, an extrinsically oriented activity. The 'like for like' phenomena dispatches with Instagram's use as a way to genuinely connect with others, or to produce or follow images that are interesting or appealing in and of themselves. Instead, its explicit purpose becomes building a following and thus increasing social status. This use of Instagram will likely appeal to users already high 
in extrinsic values: Ryan and Deci argue that individuals with strong extrinsic values are more likely to "value a partner for her or his wealth, looks, or status, in which case the connection with the partner is a means to extrinsic interests, rather than being an end in itself" (2017, p. 314). According to the values circumplex, status is an extrinsic value diametrically opposite to values of environmental care and personal wellbeing (see Figure 2.2).

The 'like for like' trend acts as a controlling factor because it is concentrated on increasing status, which relies on approval from others. Success in this goal is outside the capacity of the individual, which limits their autonomy to define their own success. Further, aspirations of status incentivise individuals to act according to the whims of others in order to win their approval, rather than express themselves freely.

\section{3 "What they want to see:" Following the demands of the 'market'}

Many participants spoke of a range of ways they experienced pressure to create content that would be approved by their followers. Instagram provides a platform where images are immediately publicly available to view, and this affects how users interact with the platform, such as demonstrated by the following comments from Georgia:

"There are so many layers to it and it can really fuck with your thinking, to see 300 people have seen this, or 1000 people follow my page, like... these people are clearly expecting something from me." -Georgia

These comments display a constraint defined by SDT as 'surveillance,' which examines the psychological impact of feeling watched, and implicitly, evaluated. According to SDT, perceptions of being watched and evaluated decrease intrinsic motivation due to its "phenomenological significance as a form of external control" (Ryan \& Deci, 2017, p. 147). This controlling factor impacts intrinsic motivation regardless of approval or disapproval:

Even when people receive positive evaluations, the knowledge that they are being evaluated can reduce their intrinsic motivations. Thus, when people post on 
Instagram with an awareness that images are there to be viewed by others (surveillance), and to receive likes and comments (evaluation), this suggests a likely perceived external control; and therefore decreased intrinsic motivation.

Some participants discussed tailoring content to what they believe their followers 'want to see,' feeling pressure to portray scenes in a way that fits the norms and perceived expectations of followers. For instance, Amy spoke of her Instagram portrayal of a holiday in Tahiti:

"In a couple of my photos you can see beautiful backgrounds but then if you turn the camera around you can see all the villagers' houses and stuff, which are- they're not in poverty but it's not anywhere near as I guess glamourous as it looks in that photo."

(Researcher:) "Why did you show the glamourous side?"

"Well to be honest it's a lot prettier isn't it, to show that side, and I think my followers would enjoy that a lot more." -Amy

Despite personally preferring the more 'real' places, Amy believed others wanted to see only the 'glamorous' side of Tahiti. Before her holiday, she had seen Instagram images of Tahiti which followed the Instagram norm Ho and Ito describe as "idealised images of affluence and attractiveness" (2019, p. 1360). She accordingly adapted her content to follow this norm with her own representation of Tahiti, interpreting the norm as audience preferences. 


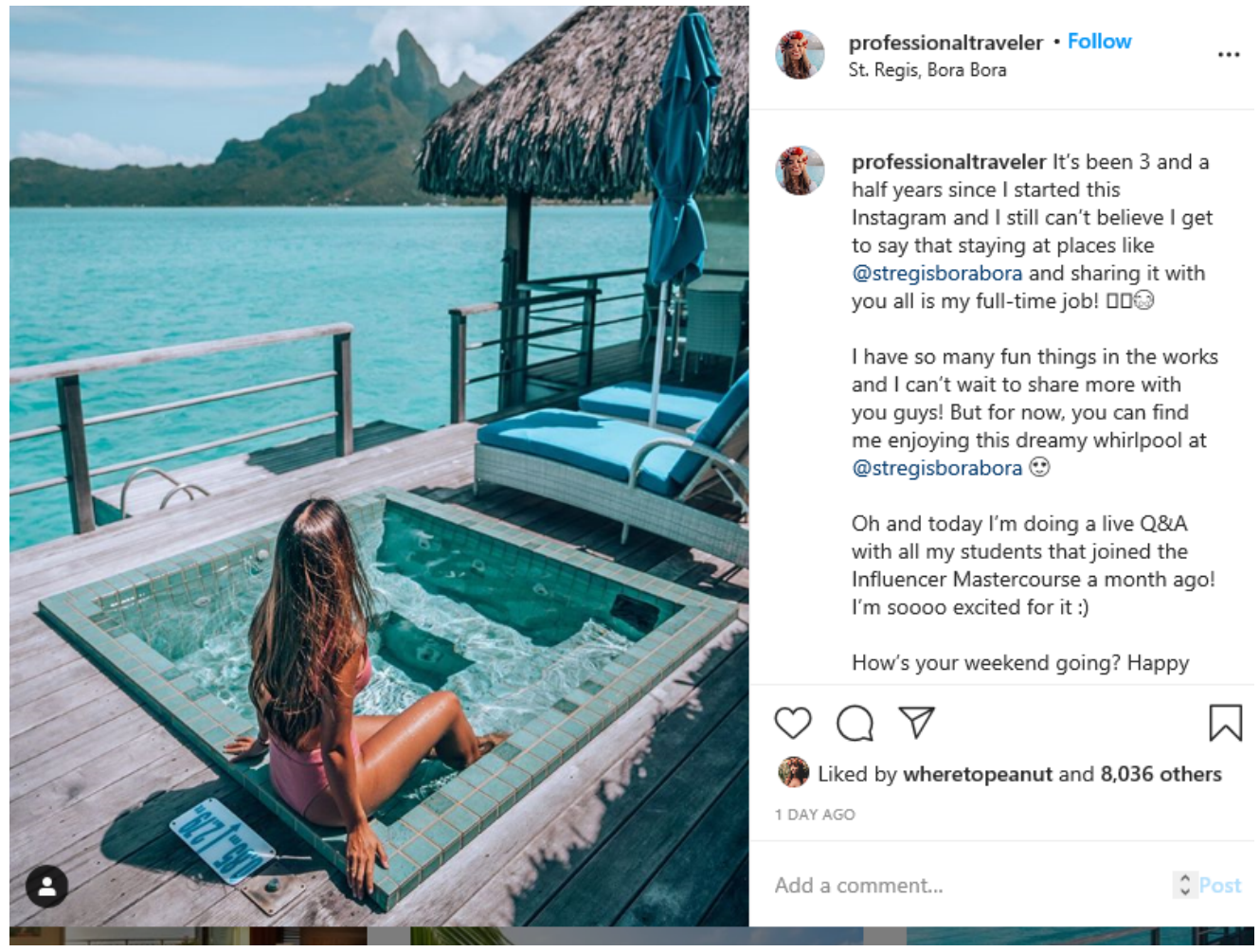

An Instagram image with the hashtag \#Tahiti. Source: @professionaltraveler

https://www.instagram.com/p/CEzs8XZg2B9/

However, what people genuinely 'want to see' may not be adequately communicated through qualitative likes. For instance, while Amy believed her followers wanted to see glamorous, idealised depictions of her holiday, participants consistently stated that seeing idealised Instagram content made them feel personally inadequate (see Chapter 5 for a full discussion on this topic). This indicates a disjuncture, in that the images people believe their followers 'want to see' in fact appear to be driving psychological discontent. Content interpreted as what people 'want to see' may, rather, simply depict themes that are more conducive to the norms and infrastructure of the platform. Images which gain more likes are socially 'endorsed' (Meixner \& Marlow, 2017), and users are thereby encouraged to create further similar content. As Instagram users seek success on the platform, the measures of which are qualitatively measured in likes and follows, they continue to create content garnered toward achieving more likes; further entrenching these norms.

SDT's theory of conditional regard can further illuminate perceived pressure to post content which will be approved by followers. Conditional regard describes when a person is only accepted or appreciated if they behave in a certain way. For instance, 
parents may 'control' the behaviour of their children by encouraging them to act in socially desirable ways. To receive their parents' support, the children "must give up autonomy to get relatedness" (Ryan \& Deci, 2017, p. 311). Following this theory, children do not have free reign to express themselves genuinely but learn to adapt their behaviour to what is deemed appropriate in their social context. Translating this to Instagram, users may feel pressure to curate an online version of themselves which matches what they perceive as socially desirable or appropriate in an Instagram context. They post what they believe their followers want to see, and in doing so lose a level of autonomy, but gain a semblance of relatedness through 'likes'. Users are thereby conditioned toward posting content which will be more 'liked' by their followers.

Through the pressure to be successful on Instagram, an experience of place is packaged into a commodity which is traded for likes online (Smith, 2018). In this marketplace infrastructure, the inherent incentive is to create the most valuable commodity. As a result, as demonstrated by Amy's comments above, Instagram users post images that will succeed in this 'marketplace' as opposed to images that portray their genuine experience. While Amy may not consciously think of the platform as that of a marketplace, this contextual background influences the way she interacts with the platform. Her priority to post what her followers will 'like' signifies a move away from using Instagram for intrinsic aims such as, for example, a creative outlet for self-expression, to more capitalist values of success and self-promotion in the Instagram marketplace. In internalizing aspirations which are dependent on the approval of others, this introduces a control, thereby limiting autonomy.

\subsection{Infrastructural incentives toward self-promotion and self-objectification}

As stated above, many participants noted that despite at times wanting to post images of scenery or friends, they refrained from doing so due to the pressure to gain 'likes.' Following the demands of the 'market,' participants consistently stated pressure to post more photos of themselves; conscious that these images would receive more "likes" than other content. Accordingly, they constructed their online lives tailored to what they believed their followers 'wanted to see' (as determined by the numbers of likes and comments the different types of content received). Billie, 
who for a period was aiming to be a full time 'influencer,' spoke of the pressure she felt to maintain her online image. She felt compelled to post self-promotional photos of herself, finding her number of followers dropped if she posted any other content.

"It just didn't fit the theme of what people followed me for so
people started unfollowing me, and I was just like ohh no that's a
bad idea, so I got caught up in that whole situation of-I just wanna
post what I want but it- I couldn't have lots of followers and
achieve that goal I guess, if I was just posting what I kind of
wanted to post." -Billie

Billie's experience exemplifies a perceived lack of autonomy. She felt pressure to post a particular form of content which did not reflect how she genuinely wished to express herself. While she wanted to post a more varied selection of photos, she posted predominantly self-promotional images of herself, following the demands of the Instagram 'market.' Like many participants, she was acutely aware of the tendency for images of oneself to receive more 'likes.' Receiving approval for posting images of oneself, as opposed to any other content, reinforces a cultural context in which self-promotion is the norm.

These pressures toward self-promotion counter research from Wang and $\mathrm{Li}$, who argue that posting to social media supports feelings of autonomy "because people can choose what and how to produce UGC" (user-generated content) (2014, p. 244). While this is technically true- there is little explicit direction around what to postthis theory ignores the more subtle social influences and contextual norms experienced by participants. No context is 'neutral', and perceived pressures to conform to norms and expectations can influence how users interact with a social platform (Jungselius, 2019), thereby potentially influencing autonomy. For instance, Chua \& Chang present a more contextual approach, analysing Instagram posts through the theory of self-presentation. According to the study, "through selfpresentation, a person pleases the audience by matching her performance to audience expectations and preferences. Additionally, self-presentation allows a person to project her performance to the ideal self" (2016, p. 191). On Instagram, users understand follower preferences through how many likes or comments an image receives. When images of themselves receive more likes, there is an incentive to 
"match audience expectations and preferences" by continuing to post further images of themselves, concomitantly participating in self-promotion. Through the perceived expectations of followers and pressure to post 'successful' images, self-expression on Instagram may adapt to fit self-promotional norms and expectations.

While photos of the self consistently gained more likes than other content, Laura, Billie and Amy all mentioned that further to this, their overall most "liked" photos were those in which they wore bikinis:

\section{"If you post a photo of yourself, you're gonna get a lot more people commenting and liking it. especially if you're looking really good, or I don't know you're wearing a bikini or something like that" -Laura}

Their experiences align with findings from a United Kingdom study, which found that almost a third of the women who participated in the study featured, using their term, 'self-objectifying content' on their Instagram (Bell et al., 2018). Selfobjectifying content was defined as images that showed $75 \%$ of the woman's body, a sexually suggestive pose, or a body part as the emphasis of the image. Content that was defined as self-objectifying consistently received more likes than other content. According to the study, "receiving more positive feedback on objectified self-images relative to non-objectified self-images indicates that such self-presentations will please the audience, thus providing motivation for presenting the self in similar ways in the future" (p. 87). For Instagram users seeing that their previously most "successful" posts are bikini photos, they can see that if they post more bikini photos, they are likely to receive more 'likes.' This presents an incentive for users to view their own value as contingent upon maintaining physical appearance, thus creating an ongoing pressure and emphasis on the extrinsic value of image. While users may outwardly conform to how they believe others perceive them, it can also internalise more deeply as self-objectification- instilling a belief that their value truly is in their physical appearance (Ryan \& Deci, 2017).

However, in counter to the growing body of research claiming the 'narcissism' of selfies and self-objectification, other research argues that such content is an expression of feminist empowerment. From this perspective, such selfies may 
contribute to autonomy, particularly when other social environments are more repressive. Murray argues for a "productive counter-reading of the "selfie," one that advances the possibility that popular forms of female self-imaging may offer the opportunity for political engagement, radical forms of community building - and most importantly, a forum to produce counter-images that resist erasure and misrepresentation" (2015, p. 491). He, alongside other researchers such as Holowka (2018) and Olszanowsky (2014), argue that through self-representations on Instagram, women are resisting traditional ideas of what is 'acceptable' and taking control of their own representations. In this way, Instagram users may find a greater sense of autonomy.

As part of this broadening of acceptability is the recent upsurge of "body positive" content (Cohen et al., 2019). This movement eschews traditional ideas of the "body perfect" (Dittmar, 2008) to celebrate types of bodies traditionally absent from media depictions. In reference to this, Jen stated:

\section{"I think now because social media has popped up a lot and because there's lots of controversy on what a good body is, I think all the discussion around self-love has definitely developed a lot more, and I think that's really good, in a way, because if social media wasn't a thing, and you were still seeing these top models on billboards and stuff, maybe that discussion wouldn't be as strong." -Jen}

From this perspective, Instagram has given a platform to show alternatives to the traditionally portrayed depictions of women in the media. As Instagram content is user-generated, this 'allows for bodies that are typically marginalised by society's dominant appearance standards to finally have a voice and be seen" (Cohen et al., 2019 , p. 1548). While existing research on values tends to depict anything related to 'image' as an extrinsic goal, it may be that gaining self-confidence in one's body image lowers extrinsic concerns about what others think. By broadening public notions of what bodies can look like, people might feel more accepted, confident, and therein experience a greater sense of autonomy. Early research shows that, in contrast to viewing images of thin-ideal bodies, viewing body-positive content was found to increase mood and body satisfaction (Cohen et al., 2019); indicating that viewing body-positive imagery may be conducive to basic human needs. 

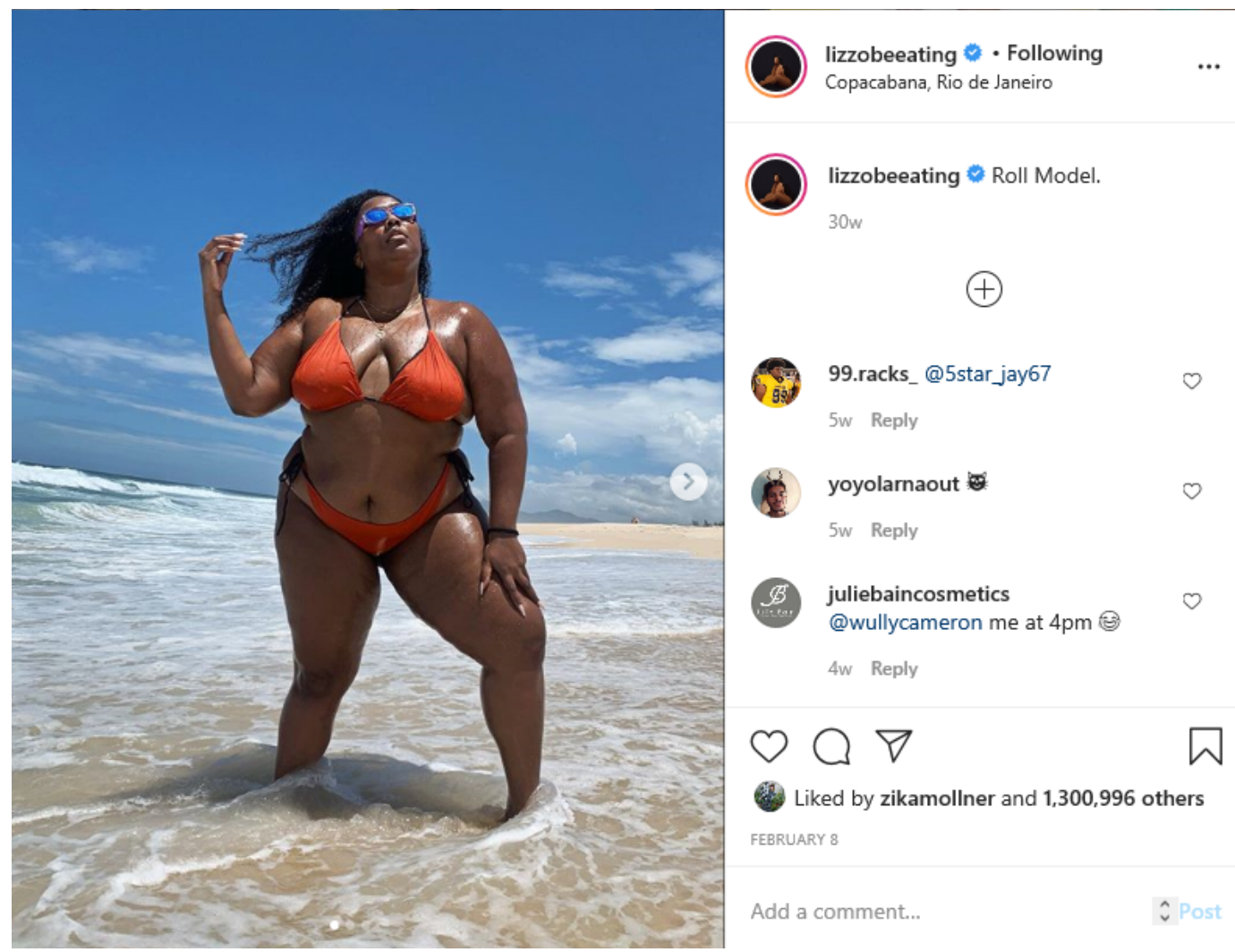

An example of 'body positive' content on Instagram. Source: @lizzobeeating https://www.instagram.com/p/B8RmA6ahHTq/

8.5 "Do it for the Gram:" from intrinsic experiences to extrinsic representations

Many participants stated examples of experiences they constructed for the primary motivation of posting a photo on Instagram. For instance, participants decided what/where they chose to eat, why/where they decided to walk, and what they prioritised when travelling according to what would look appealing on Instagram. The following quote from Amy describes her experience of a music festival:

"Oh my god we took so many photos, and we were like on the balcony and then we were on the couch then we were standing by a window, and we would flick through them and be like OK I like this angle, but I don't like my hair like that, or OK I've gotta sit like this, and OK can you take a few more, and it was like intense. And it was all for Instagram. It was not like we were even having fun, or like for memories- it was 100\% only point of it was to post on Instagram. Which we did. We got great photos." -Amy 
In these situations, the inherent (intrinsic) value of the described activities (eating, travelling, live music) diminishes, overtaken by the extrinsic motivation of representing that experience to others. SDT states that "doing an interesting activity because any outcome is expected to be contingent upon it runs the risk of decreasing intrinsic motivation, and in an attribution sense, demeans the primary activity" (Ryan \& Deci, 2017, p. 129). Therefore, participating in an activity primarily to post it on Instagram prioritises an outcome (gaining online gratification), which may detract from the experience itself. An intrinsic motivation thus evolves to become extrinsic as activities previously done for enjoyment become provisioned upon being 'worthwhile' according to external measures. In each case, users are practising and reinforcing importance on extrinsic motivations, which, according to the structure of values (see Section 2.5), directly conflicts with the intrinsic values associated with both mental and ecological wellbeing.

Participants stated experiences of feeling pressure to maintain an online presence in terms of both quality and quantity of posts, and to uphold a representation of an exciting/productive/successful life. Many participants stated that they had felt pressures relating to maintaining an online presence in the past, but did not, at least to the same extent, at the time of the interview. Krista spoke of how she deleted her Instagram account and subsequently realised the pressures she had perceived coming from others were self-imposed:

"I think when I deleted my Instagram I was like'oh shit, how are people gonna know that you're like, alive? How are people gonna know what you're up to?' All that kind of shit. But I got to a point where I was like, people don't need to know that. And I think I was definitely at a point where I was like taking photos or videos coz in my head I'm like 'I'm gonna put this on Instagram. 'And it wasn't for me. It was to show other people that I was having fun, or I was doing something. And I think having removed that out of my life I really had a think about why I'm creating content, who I'm actually creating it for, is it good for me.' -Krista

Krista internalised a perceived pressure to project her activities to an online audience, and further, to participate in more offline activities in order to augment the 
online representation of her life. This may be an example of, described in SDT, seeking to maintain self-worth by "perform(ing) in ways that are valued by a reference group to which they do or would like to belong" (Ryan \& Deci, 2017, p. 168). The internalised pressure to prove her worth to others on Instagram dissipated when she deleted it, taking away the internalised control of her followers' expectations.

Ryan \& Deci (2017) further state that introducing external rewards to an activity causes engagement with the activity to decrease. In an Instagram context, it is possible that posting about an experience becomes preoccupied primarily with the reward of 'likes;' as opposed to motivated through intrinsic engagement in the activity itself. For Krista, deleting Instagram and thus removing any internalised pressure to gain 'likes' allowed her to regain intrinsic interest in activities, and further, regain a sense of autonomy as the perceived control of expectations of followers no longer existed.

\subsection{Influencers as Cultural Messengers}

The aptly named 'influencers' largely shape the culture of Instagram. Influencers are "defined by their ability to influence, shaping consumer behaviour and perceptions in a specific field or market" (Newlands \& Fieseler, 2020, p. 168). According to SDT, cultural norms are set by cultural messengers such as religious leaders, politicians, and celebrities (Ryan \& Deci, 2017). In the context of Instagram, this can also be extended to influencers, who have a reach of many thousands or even up to hundreds of millions of followers.

Participants demonstrated mixed feelings about the values and ideals promoted by influencers. Several participants mentioned frustration that most influencers focus on self-promotion and materialistic themes when they could use their platforms to raise awareness for social or environmental issues. Others, attracted to the lifestyles portrayed by influencers, felt that Instagram offered a pathway to success, away from the mundaneness and responsibilities of their real-life experiences. Instagram influencers offer a vision of the 'good life,' which appears attainable to anyone who can 'brand' themselves successfully enough (Khamis et al., 2017; Loukianov et al., 
2020). Influencers' depiction of success has tended to involve beauty, travel, and fame; portraying an idealised lifestyle which promotes these values, which viewers of this content then internalise as aspirations. This was exemplified by Laura:

"To be instagram famous meant that you didn't have to go to uni anymore and brands would just send you stuff and you can just travel the world, and it will be that life that you see on Instagram." -Laura

Influencers provide a form of escapism; a route to the 'good life' that appears accessible to anyone who can build enough followers. The content Laura speaks of is pervasive across the platform: In a study of 200 randomly selected images on Instagram with the caption '\#goodlife,' the authors interpreted three themes. Related to Laura's comments, one of the most pervasive themes was the "world traveller," a well-off, fashionable "traditionally beautiful young woman" in exotic locations. Another prevalent theme was "the self-made affluent entrepreneur," which included portrayals of lavish lifestyles, wealth, and (generally masculine) ideals of beauty. Lastly, with significantly less quantity of posts and less engagement (i.e. likes), was "shared experiences," which appears to be a catch-all for more intrinsic experiences such as creative pursuits and connection to nature (Loukianov et al., 2020). The vast majority of content examined thereby promoted the ideals of the 'good life' as luxurious and glamorous, with less attention given to intrinsically enjoyable pursuits.

Through collaboration with commercial brands for mutual economic gain, influencer depictions of the 'good life' generally embody themes of wealth, luxury and beauty. As stated by SDT, individuals are not only recipients of such cultural messages but play an active part in it "as participants in a cultural community" (Ryan \& Deci, 2017, p. 564). Within the context of Instagram, Loukianov et al. similarly state: "Conceptualising Instagram as a social conversation highlights the necessity to pay attention not only to how the conversation shapes the world for others but also what it requires us to do to actively participate" (2020, p. 12). The values of wealth, image, and fame espoused by influencers are admired, internalised, practised and therefore perpetuated by many individuals within the cultural community of Instagram. Exposure to influencers' blend of public persona and commercial intent may encourage individuals to see themselves in a similarly commodified nature. For 
instance, Page suggests that social media positions identity as "a product to be consumed by others, and an interaction which treats the audience as an aggregated fan base to be developed and maintained in order to achieve social or economic benefit" (Page, 2012, p. 182). Influencers use Instagram to self-brand for commercial success, and in doing so, set norms for self-branding that other users may adopt even when using Instagram as a non-commercial hobby.

These concepts are directly extrinsic on the values circumplex (see Figure 2.2), and oppose values related to environmental care. Further, research from Kasser (2002b) shows materialistic values, such as regularly promoted by influencers, conflict with feelings of autonomy. As he states, while consumer societies regularly promote aspiring to live the high-luxury lifestyles of the most 'successful,' "a strong focus on the pursuit of wealth, fame and image undermines the satisfaction of needs for authenticity and autonomy" (2002b, p. 73). This is because focussing on the materialistic pursuits espoused by influencers and other such role models of consumeristic cultures is distracting from more fulfilling, intrinsic pursuits, and further is a "fundamental alienation from what is truly meaningful" (2002b, p. 3). Influencer ideals of fame, wealth and image, thereby, depict ideas of happiness which are on the whole misleading, creating norms and standards which followers feel a need to aspire/live up to, which distract from what is truly meaningful and satisfying.

\subsection{Influencer commercialism}

Many participants also spoke of the direct harm they felt was caused by influencers promoting commercial products. Several participants mentioned frustrations at the way the platform was commodified through influencer product placement, particularly in its capacity to have a detrimental impact to themselves and others at their most vulnerable.

"Just to vulnerable people saying, 'if you want to look like me this is what you have to do.' Or 'If your tummy's not flat then you're not beautiful,' sort of thing, which-I've fallen for it before coz I don't have a flat tummy, so seeing like Kim Kardashian promoting like a-it's not just Kim Kardashian, she's just the only person I can think of right now-like promoting a tea that makes your stomach 
flat, you're kind of like 'oh ok so that's what I need to do to be like you, to be beautiful and stuff.' You know?" -Amy

Users' issues with influencer commercialism are widespread: one study found that $44 \%$ of the UK public thought influencer marketing was "damaging" to society (Stewart, 2018). Harrison argues that "consumers develop fictitious emotionallybonding relationships with endorsers that strongly resemble real friendships," which can then be exploited for commercial gain (Harrison, 2018, p. 399). Thereby, users searching for relatedness are told they must buy products in order to gain acceptance or approval; detrimental to both relatedness and autonomy.

While users may be fully aware of the constructed, commodified nature of the lifestyles promoted on Instagram, they may still be susceptible to commercial influence. While Amy, as mentioned above, bought the tea Kardashian promoted, she also showed cognisance of the background to the commercial images:
"Coz the influencers are getting paid to do it, they don't really think half the time-like Kim Kardashian doesn't drink a tea that flattens her stomach. She has professional chefs and a professional personal coach that gets her the body she wants. It's not from a tea that cost \$20, you know? But for someone who's vulnerable already, to see that, then they would probably buy into it. "-Amy

Despite a conscious understanding of the commercial manipulation involved, it nevertheless influenced Amy. As she mentions, the commercial intent may be particularly powerful when viewers are feeling vulnerable. Considering the typical Instagram feed contains highly edited idealised content, there are likely to be such vulnerabilities which emerge from upward social comparison and feelings of relative inadequacy (see Section 5.3). As stated by Hamilton, marketing infrastructure aims "to help us manufacture ideal selves and to supply the goods to fill the gap between the actual and the ideal" (2005, p. 13). Users who may be feeling vulnerable through thwarted competence may experience increased susceptibility to influencer messages signalling, as Amy referenced above, "that's what I need to do to be like you." Therefore, within Instagram infrastructure, there is both the capacity to thwart competence and thereby prompt vulnerabilities, and commercial messages which 
state that such vulnerabilities can be overcome with the purchase of (primarily image-based) products.
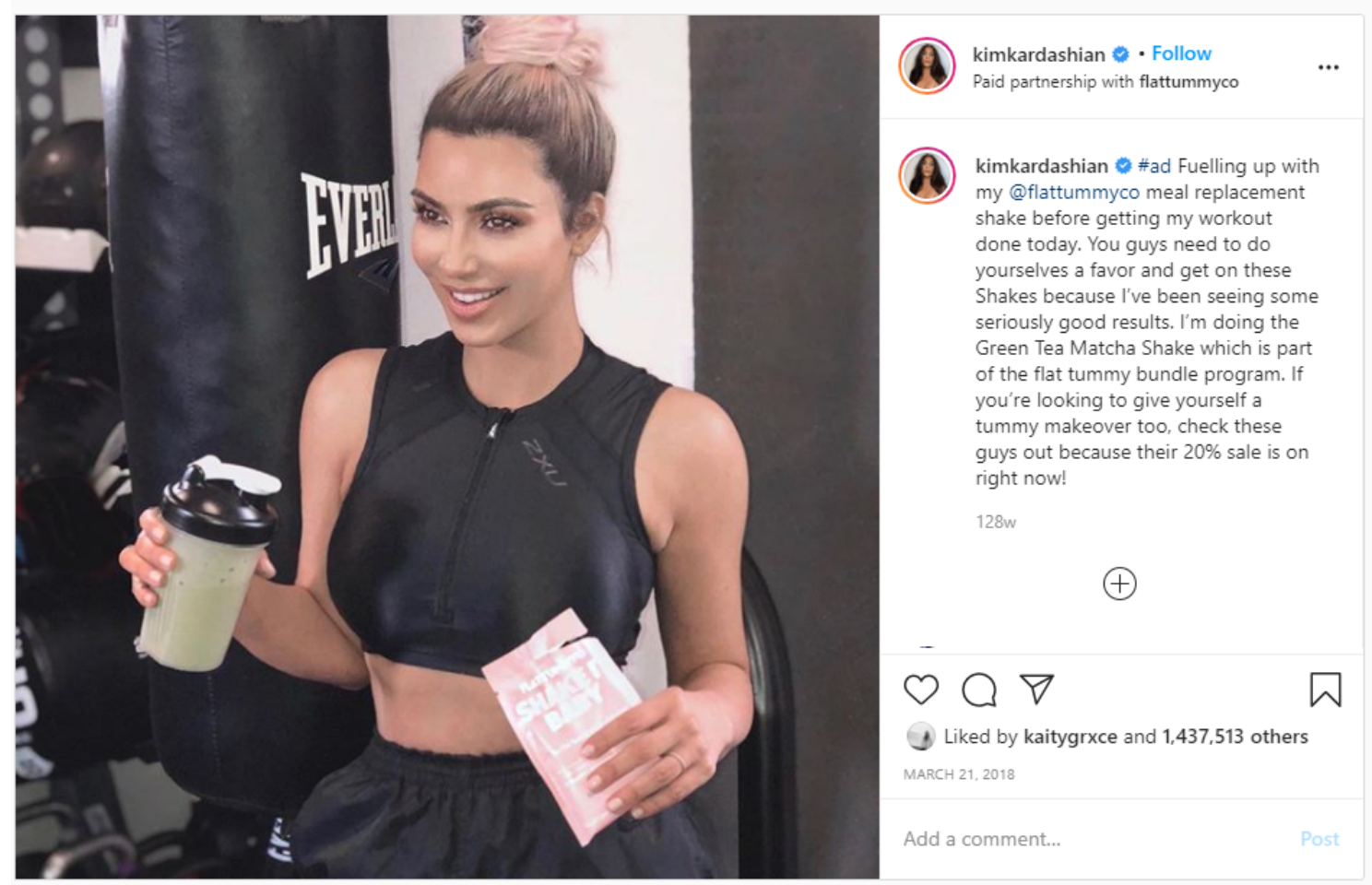

One of Kim Kardashian's Instagram posts promoting slimming tea.

Source: @kimkardashian https://www.instagram.com/p/BgkAznclyTt/

Several participants stated experiences similar to Amy's: During our discussions, they took a philosophical view of influencers, fully cognizant of the constructed nature of their profiles and resistant to their commercial messages. However, they also stated that there were times in which they were not able to have such an outlook and may be affected by such content, buying the promoted product or feeling psychological detriment. Many participants also expressed concern for other users seeing influencer content who may be more vulnerable, younger, or less savvy to the commercial intent.

\subsection{Targeted advertisements}

Participants stated an awareness of being targeted for advertisements, with many sharing experiences when they were amazed to receive advertisements directly related to a product they had been recently thinking about. This demonstrates the 
still-novel pervasiveness of micro-targeting. Micro-targeting involves tailoring online advertisements to appeal to individuals, according to data analytics gained through previous online activity. Businesses can either only target demographics who are more likely to engage, or they can highlight different features of their product according to what they know will appeal more to each individual (Winter et al., 2020).

Participants were highly aware of advertisements on Instagram, with reactions ranging from interest in the tailored products, to full dismissal, to concern over how well the advertisements 'knew' them. Caro stated that it is difficult to completely ignore Instagram advertisements:

\section{"I try to filter ads out of my brain when I'm looking through things, so, I try to like-I don't know it's like wearing a tinfoil hat, like I'm not affected by these ads, you know what I mean? But we probably are anyway, coz we're seeing them..." -Caro, GII}

While Caro tries to ignore the advertisements she sees on Instagram, she acknowledges they may still be impacting her, perhaps subconsciously. This instinctive feeling is backed up by literature: the advertising industry, existing to create desires for products, is a core driver of materialism (Kasser, 2016; Richins, 2017). Ramifications are substantial in terms of mental wellbeing: A study of one million Europeans in 27 nations examined data of life satisfaction and advertising expenditure over 3 decades. They found that when advertising expenditure of a country increased, life satisfaction significantly declined, and vice versa (Michel et al., 2019). These findings align with SDT research that increased materialism (fostered by pervasive advertising stimulating desires for products), conflicts with basic need satisfaction, and, therefore, wellbeing (Kasser, 2002b; Ryan \& Deci, 2017).

Kasser (2016) states that a key pathway to reducing materialism is to limit people's particularly children's- exposure to advertising. On Instagram, however, advertisers have increased access to people; and in particular to adolescents, with $43.1 \%$ of Instagram's billion users under 25 years old (Statista, 2020). The quantity of advertising on Instagram is substantial, with the platform gaining U.S\$9.45 billion in 
advertising profits in 2019, and projections for that revenue to double in 2021 (Statista, 2020). Alongside increased exposure to potential customers through targeted advertisements, corporations also have few regulatory restrictions. This is exemplified by a study from Barry et al. (2016), which found that their fictitious underage Instagram users as young as 13 had free access to following alcohol brands, and in doing so received 362 alcohol-related advertisements over thirty days. Despite many nations having regulations restricting alcohol brands from advertising to adolescents, on social media these brands were able to bypass these regulations, with considerable access to, and direct interaction with, underage Instagram users. Instagram's provision of increased, unregulated access to adolescents directly conflicts with recommendations toward reducing materialism.

According to Ryan and Deci (2010), the advertising industry is a controlling influence that limits autonomy, as it is based in the idea that "without having more and more one cannot be successful or happy" (p. 46). Similarly to previously discussed impacts of influencers (see Section 6.6), these cultural messages on what it takes to be happy can be internalised by individuals within the culture, causing individuals to aspire toward extrinsic goals which will be less likely to actually contribute to wellbeing (Kasser \& Ryan, 2001; Ryan \& Deci, 2017). The power of Instagram to promote advertising, outside of regulatory scrutiny, is counter to aims of mental and ecological wellbeing; and urgently deserves further academic and political attention.

\subsection{Conclusion}

Instagram encompasses a set of norms, expectations, pressures and precedents. Some participants felt pressure to be 'successful' online, tailoring content to what they understood would gain more likes. In doing so they were influenced to produce more self-promotional and self-objectifying content. These incentives toward selfpromotional and self-objectifying behaviour are closely tied to extrinsic values; with emphasis on image, status and fame. However, concepts of self-objectification are nuanced and depend on other contextual factors or motivations, such as whether the images are to promote empowerment and body positivity, or to conform to conventional ideals of attractiveness in return for 'likes.' 
In aiming to maintain or enhance an online self-image, many offline activities became primarily motivated by the will to gain Instagram content. Offline experiences thus become opportunities for online self-promotion, curating a selfbrand that would be successful in the Instagram marketplace. This detracts from the intrinsic value of an experience, as it becomes primarily about portraying the experience to others in order to increase one's online status.

Instagram, itself operating as a successful for-profit enterprise in a neoliberal economy, encompasses commercial values within its infrastructure. Its most 'successful' users are 'influencers,' who are the most popular users as rated by follower count, and who use these followers as a 'market' from which they can make economic gain. Typical ideals of success established by influencers include emphases on fame, popularity, attractiveness, and perpetual travel, portraying these values as associated with happiness and 'the good life.' However, these aspirations are intertwined with capitalist values, as the influencers espousing these messages are pursuing economic gain through their self-brand. Influencers, as cultural messengers, spread these values to their followers, who may internalise them despite having no economic incentive to do so.

Self-promotional values heavily align with extrinsic values, with both primarily concerned with image, status and fame. Incentives toward, and normalising, selfpromotional behaviour are antithetical to the intrinsic, self-transcendent values associated with mental and ecological wellbeing. The danger of Instagram's capacity to influence cultural values is significant, with over a billion users globally and minimal regulatory controls. Participant experiences demonstrate an incentive to embrace influencer values of self-promotion, indicating a cultural shift further along the values circumplex to stronger extrinsic values.

Further, direct commercialism is pervasive on Instagram, through both influencer product placement and targeted advertising. These commercial messages may be particularly powerful when users are feeling thwarted competence through comparing themselves to idealized images on the platform. Further, the lack of regulatory mechanisms allows advertisers significant access to adolescents. The quantity of largely unregulated advertising on Instagram is likely to increase 
materialistic desires, negatively affect users' mental health, and runs counter to ecological goals. 


\title{
9. Conclusion
}

\author{
"We are, in other words, in the midst of a natural experiment with \\ mankind's psychological makeup" (Aagaard, 2020, on the pervasiveness \\ of online activity).
}

\subsection{Overview}

Humankind is in the midst of multiple ecological crises. The world's top climate scientists state that there must be "unprecedented changes in all aspects of society" within the next decade in order to avoid catastrophic climate change (IPCC, 2018). In this thesis, I argue that such a transformation requires an examination of the prominent extrinsic Western cultural values that have led to and still perpetuate ecological crises. A large body of research demonstrates links between intrinsic values, environmentally responsible behaviour, and mental wellbeing; while extrinsic values are correlated with lower levels of wellbeing, less environmental concern, and a higher carbon footprint. Self Determination Theory (SDT) states that there are three universal human needs, which, if not met, lead to more extrinsic life goals, with associated mental and ecological impacts.

This study aimed to explore how participants' experiences of Instagram demonstrate incentives toward intrinsic or extrinsic values, using existing literature to explore implications for mental and ecological wellbeing. Emergent themes related to each universal human need: Connection, which is discussed in the context of the basic human need of relatedness; social comparison, discussed in terms of the basic human need of competence; and infrastructural incentives which influence how participants use the platform, which relates to the third basic human need of autonomy. Within these themes, while there were instances in which Instagram appears to contribute to basic human needs and promote intrinsic values, participant experiences indicate many ways in which the infrastructure of the platform can lead to thwarted basic human needs, and an exacerbation of extrinsic values. Findings are summarised below: 


\subsection{Relatedness}

Instagram, as a 'social' media, is premised upon offering users connectivity. Indeed, interviews with participants and existing literature indicate that keeping up/connecting with others is a primary reason for using the platform. This study also found that there are situations in which Instagram can help to fulfil a sense of relatedness for people who do not otherwise feel connected to an offline community. Further, participants stated the usefulness of being able to maintain relationships through easily accessible regular contact, such as tagging others in memes. However, participant interviews also highlighted other situations in which Instagram can be detrimental to overall relatedness. Participants stated feeling that Instagram did not satisfy a need for connection, and that even when they felt overloaded from Instagram interactions they did not feel a sense of relatedness. Participants' interactions on Instagram often centred on gratification in how one looks; which promotes the extrinsic value of 'image.' Further, many participants stated frustrations at Instagram taking precedence in offline interactions; either when their friends wanted to post their interaction on Instagram, or when they were browsing Instagram while simultaneously engaging in a conversation. This example, in particular, demonstrates an increased extrinsic motivation of representing an experience to others, to the detriment of intrinsic enjoyment of the interpersonal experiences themselves.

\subsection{Competence}

Participants spoke of Instagram as displaying a 'highlight reel' of one's life. As users can create an idealised version of their 'best' selves, viewers seeing these images can feel incompetent in comparison. Participants stated that they feel inadequate through comparing themselves to the polished personas they viewed on Instagram. This indicates thwarted feelings of competence. Research has demonstrated that regularly comparing oneself to those seen as 'better' can lead to materialistic tendencies in order to repair feelings of competence. Thus, the Instagram norm of creating idealised content encourages materialistic tendencies as users attempt to 'keep up' with an unattainable idea of reality. 


\subsection{Autonomy}

The infrastructure of Instagram sets norms and expectations for users to follow. While each user has a uniquely tailored experience of Instagram, the platform infrastructure itself is not neutral and therefore impacts users' experiences. Participants stated that they felt pressure to conform to expectations of followers, and that they consciously posted content which they believed would be more successful in gaining 'likes.' Consequently, following the will of the 'market,' participants posted more self-promotional images. Many participants also stated that they would seek out experiences primarily motivated by creating successful Instagram content. These experiences demonstrate participants feeling pressure to engage in activities, rather than for their intrinsic worth, for the extrinsic motivation of portraying it to others in return for approval in the form of 'likes.' Some participants indicated an internalization of the ideals of the 'good life' promoted by influencers, often centred around materialistic themes which undermine basic need satisfaction.

Participants also stated frustration with the directly commercial aspects of Instagram, in terms of both product placement from influencers and targeted advertising. Both of these commercial imperatives act as a controlling factor, sending false messages to users that buying a perpetual flow of products will lead to happiness. As well as being detrimental to autonomy, consumerism is directly associated with ecological damage (Hamilton, 2005; Jackson, 2017) and lower levels of wellbeing (Brown \& Kasser, 2005; Kasser \& Kanner, 2004).

\subsection{Bringing it all together}

Examining participants' experiences of Instagram, there are multiple ways the platform appears to affect values. Firstly, Instagram contains direct promotion of extrinsic values such as fame, wealth, image, and materialism, through norms set and perpetuated by influencers and internalized and practised by other users of the platform. Further, through incentives to gain social approval on Instagram, participants indicated a shift from situations in which they intrinsically enjoy a variety of life experiences to an extrinsic motivation to represent those experiences 
to others in order to gain social approval. Participants discussed how social interactions were less satisfying when friends were preoccupied with posting to, or browsing through, Instagram. Experiences such as eating in restaurants or travelling also frequently became motivated by representing them on Instagram, to the detriment of intrinsic satisfaction of the experience. Feasibly, any intrinsic hobby or activity that can be photographed can become a way to increase social capital on Instagram, therein reinforcing an emphasis on social approval and selfrepresentations, which is, by definition, an extrinsic orientation. Moreover, according to participant discussions, Instagram frequently thwarts the basic human needs of relatedness, competence and autonomy. Thwarting these needs is likely to lead to an increase in extrinsic values, as individuals search for a compensatory strategy which will offer some temporary semblance of need satisfaction (Deci \& Ryan, 2002).

These results indicate ways in which Instagram may be encouraging extrinsic values; through directly promoting extrinsic values, intrinsic experiences becoming extrinsic representations of experiences, and through thwarting basic human needs. These findings are cause for concern, as culturally sanctioned extrinsic values are fundamentally incompatible with avoiding catastrophic climate change.

\subsection{Implications for mental and ecological well-being}

According to the values circumplex, the Instagram incentives toward extrinsic values directly conflict with the values associated with environmentally responsible behaviour. A necessary element of mitigating climate change will be to minimise societal messages encouraging materialism and gaining social approval through selfenhancement, and instead, employing more consistent messaging promoting values of collective and environmental care. While it is daunting how much needs to change, values offer a clear way forward. Pinpointing the link between mental and ecological wellbeing indicates that responding to the challenges of climate change does not necessitate a sacrifice to a quality of life. It could instead mean an increase in quality of life- if quality is measured by subjective wellbeing as opposed to material wealth. Through culturally nurturing intrinsic values, there can be flourishing people and a flourishing planet. 


\subsection{Limitations and Future Directions}

Understanding how social contexts promote values is an enormously complex and nuanced undertaking, which this thesis only begins to explore. The different potential angles or focusses one could take are effectively infinite. This study is one small example of a much broader conversation on Western cultural institutions and their mental and environmental outcomes. This research points to an issue of massive scale with no easy solution: The shifting of an entire cultural practise of value-based messaging. This shift, while necessary, is vastly complicated; intertwined with the global economic systems relying on mass consumption. Perpetually increasing trends of consumption is one of the "central foundations on which twenty first century international capitalism is based" (Senker, 2011, p. 1016). The complexities of the deep-seated cultural belief in the necessity of economic growth is well-established in decades of literature; and though clearly a driver of extrinsic, consumerist messaging and a barrier to change, a deeper analysis of the structural drivers of extrinsic messaging was beyond the scope of this thesis.

While the shift from extrinsic to intrinsic cultural messaging may seem overwhelming or unrealistic, the world's leading climate experts have stated the necessity of societal transformation (IPCC, 2018; see Section 1.1). Therefore, considerable efforts in research, regulation and civil society need to work toward critiquing social contexts which promote extrinsic values, and focus on intrinsic values instead. The ultimate 'future direction' is moving toward a culture that encourages the intrinsic values necessary for flourishing people on a flourishing planet.

The links between Instagram, values and the environment are, as far as I can see, unprecedented in academic research. Therefore, this study is predominantly exploratory, laying out the links and preliminary findings which can then be built upon through research of larger scope or varied contexts.

This study does not claim to be representative or conclusive; to do so would require far greater sample sizes and even then may be unattainable, considering the uniquely tailored experience each Instagram user has. My sample size was relatively small 
and homogenous, made up of participants aged 17-26 from urban Aotearoa New Zealand. It was also limited to interviews as the sole research method, as I chose to focus on the in-depth experiences of a relatively small number of participants in order to explore their experiences of Instagram at a qualitative level. Through choosing this route, I necessarily ignored other avenues also worthy of research, such as content analysis, quantitative surveys, or experimental psychology. These other approaches remain valuable areas to explore. I have pinpointed three key areas of further research:

Firstly, there is a multitude of research necessary to further understand the links between social contexts (including, but not limited to, Instagram) and values. It would be valuable to gain further perspectives to see if the issues raised here differ according to geography, age, gender, ethnicity or socio-economic status. Further, while the themes in this thesis centre on Western values in a Western context, it would be valuable to gather research on whether people from other cultures experience Westernizing influences through social media. Moreover, research using other methods such as experimental psychology could contribute to understanding how environments such as Instagram affect immediate behaviour (such as using the priming methods discussed in Section 2.7).

Secondly, a critical area of research beyond the scope of this thesis involves how to work toward coming up with solutions for these issues. Data collection methods including high-level regulatory bodies and with people from inside the social media industry will be crucial in order to understand potential ways forward to create and regulate technology infrastructure which understands and limits detrimental impacts to mental and ecological wellbeing.

Thirdly, considering the magnitude of the changes in social contexts required, another useful area of research would be to experiment with local interventions that can suppress materialism in individuals. This research could have a more immediately achievable positive impact; though, as discussed in preliminary work in this area by Kasser (2016), system-level change is still necessary in order to achieve the breadth of change required. 
All in all, this thesis is an exploratory examination of how the social context of Instagram affect values, and what this means for mental and ecological wellbeing. This study indicates that Instagram contains infrastructural incentives toward further extrinsic values, which run counter to societal goals concerning mental and ecological wellbeing. These findings are cause for concern and deserve significant further academic and regulatory attention. However, these findings also reiterate the links between values and environmental and mental wellbeing; demonstrating that if these extrinsic incentives can shift to intrinsic, there are ways forward toward creating a culture of thriving people on a thriving planet. 


\section{References}

Allen, M. (2017). Sean Parker unloads on Facebook: "God only knows what it's doing to our children's brains." Axios. https://www.axios.com/sean-parker-unloads-onfacebook-god-only-knows-what-its-doing-to-our-childrens-brains-1513306792f855e7b4-4e99-4d60-8d51-2775559c2671.html

Barry, A. E., Bates, A. M., Olusanya, O., Vinal, C. E., Martin, E., Peoples, J. E., Jackson, Z. A., Billinger, S. A., Yusuf, A., Cauley, D. A., \& Montano, J. R. (2016). Alcohol marketing on Twitter and Instagram: Evidence of directly advertising to youth/adolescents. Alcohol and Alcoholism, 51(4), 487-492. https://doi.org/10.1093/alcalc/agv128

Bauer, M. A., Wilkie, J. E. B., Kim, J. K., \& Bodenhausen, G. V. (2012). Cuing consumerism: Situational materialism undermines personal and social well-being. Psychological Science, 23(5), 517-523. https://doi.org/10.1177/0956797611429579

Bell, B. T., Cassarly, J. A., \& Dunbar, L. (2018). Selfie-Objectification: Self-objectification and positive feedback ("likes") are associated with frequency of posting sexually objectifying self-images on social media. Body Image, 26, 83-89. https://doi.org/10.1016/j.bodyim.2018.06.005

Blommaert, J. M. E., \& Varis, P. K. (2014). Conviviality and collectives on social media: Virality, memes and new social structures.

https://research.tilburguniversity.edu/en/publications/b70d0aeb-48a4-4ddf-88839652db9a92da

Boer, M. M., Resco de Dios, V., \& Bradstock, R. A. (2020). Unprecedented burn area of Australian mega forest fires. Nature Climate Change, 10(3), 171-172. https://doi.org/10.1038/s41558-020-0716-1

Boyd, A. (2016). Beautiful trouble: A toolbox for revolution. New York: OR Books. 
Brown, K. W., \& Kasser, T. (2005). Are psychological and ecological well-being compatible? The role of values, mindfulness, and lifestyle. Social Indicators Research; Dordrecht, 74(2), 349-368. http://dx.doi.org/10.1007/s11205-004-8207-8

Brown, K. W., Kasser, T., Ryan, R. M., Alex Linley, P., \& Orzech, K. (2009). When what one has is enough: Mindfulness, financial desire discrepancy, and subjective well-being. Journal of Research in Personality, 43(5), 727-736.

https://doi.org/10.1016/j.jrp.2009.07.002

Burroughs, J. E., \& Rindfleisch, A. (2002). Materialism and well-being: A conflicting values perspective. Journal of Consumer Research, 29(3), 348-370.

https://doi.org/10.1086/344429

Butler, R. (1993). Effects of task- and ego-achievement goals on information seeking during task engagement. Journal of Personality and Social Psychology, 65(1), 18-31. https://doi.org/10.1037/0022-3514.65.1.18

Casale, S., \& Fioravanti, G. (2018). Why narcissists are at risk for developing Facebook addiction: The need to be admired and the need to belong. Addictive Behaviors, 76, 312-318. https://doi.org/10.1016/j.addbeh.2017.08.038

Ceballos, G., Ehrlich, P. R., \& Dirzo, R. (2017). Biological annihilation via the ongoing sixth mass extinction signaled by vertebrate population losses and declines. Proceedings of the National Academy of Sciences of the United States of America, 114(30), E6089-E6096. https://doi.org/10.1073/pnas.1704949114

Center for Humane Technology. (2019). The opposite of addiction. [Audio podcast]. https://www.humanetech.com/podcast/8-the-opposite-of-addiction

Center for Humane Technology. (2020). The stubborn optimist's guide to saving the planet. [Audio podcast]. https://www.humanetech.com/podcast/18-the-stubbornoptimists-guide-to-saving-the-planet 
Charmaz, K. (2008). Constructionism and the grounded theory method. In Handbook of constructionist research (pp. 397-411). Guilford Press.

Chotpitayasunondh, V., \& Douglas, K. M. (2018). The effects of "phubbing" on social interaction. Journal of Applied Social Psychology, 48(6), 304-316. https://doi.org/10.1111/jasp.12506

Chua, T. H. H., \& Chang, L. (2016). Follow me and like my beautiful selfies: Singapore teenage girls' engagement in self-presentation and peer comparison on social media. Computers in Human Behavior, 55, 190-197. https://doi.org/10.1016/j.chb.2015.09.011

Cohen, R., Fardouly, J., Newton-John, T., \& Slater, A. (2019). \#BoPo on Instagram: An experimental investigation of the effects of viewing body positive content on young women's mood and body image. New Media \& Society, 21(7), 1546-1564. https://doi.org/10.1177/1461444819826530

Conger, J., \& Singg, S. (2020). Should you "do it for the 'gram?" A review of the psychological and sociocultural effects of social media. Journal of Clinical Review \& Case Reports, 5(1). https://doi.org/10.33140/JCRC.05.01.09

Corbin, J. M., \& Strauss, A. L. (2008). Basics of qualitative research techniques and procedures for developing grounded theory. (3e [ed.] / Juliet Corbin, Anselm Strauss.). SAGE.

Crompton, T. (2010). Common cause: The case for working with our cultural values. WWFUK. https://assets.wwf.org.uk/downloads/common_cause_report.pdf Crompton, T., \& Kasser, T. (2009). Meeting environmental challenges: The role of human identity. WWF-UK. https://talk.eco/wpcontent/uploads/meeting_environmental_challenges_tthe_role_of_human_ident ity.pdf 
Davidov, E., Schmidt, P., \& Schwartz, S. H. (2008). Bringing values back in. Public Opinion Quarterly, 72(3), 420-445. https://doi.org/10.1093/poq/nfn035

de Vries, D. A., \& Kühne, R. (2015). Facebook and self-perception: Individual susceptibility to negative social comparison on Facebook. Personality and Individual Differences, 86(C), 217-221. https://doi.org/10.1016/j.paid.2015.05.029

Deci, E. L., \& Ryan, R. M. (2002). Handbook of self-determination research. University of Rochester Press.

Dehaene, S., Naccache, L., Clec'H, G. L., Koechlin, E., Mueller, M., Dehaene-Lambertz, G., Moortele, P.-F. van de, \& Bihan, D. L. (1998). Imaging unconscious semantic priming. Nature, 395(6702), 597-600. https://doi.org/10.1038/26967

Diffenbaugh, N. S. (2020). Verification of extreme event attribution: Using out-of-sample observations to assess changes in probabilities of unprecedented events. Science Advances, 6(12), eaay2368. https://doi.org/10.1126/sciadv.aay2368

Dittmar, H. (2008). Consumer culture, identity and well-being: The search for the "good life" and the "body perfect." Psychology Press.

Dittmar, H., Bond, R., Hurst, M., \& Kasser, T. (2014). The relationship between materialism and personal well-Being: A meta-analysis. Journal of Personality and Social Psychology, 107(5), 879-924. https://doi.org/10.1037/a0037409

Edwards, E. B., \& Esposito, J. (2018). Reading the black woman's body via Instagram fame. Communication, Culture and Critique, 11(3), 341-358. https://doi.org/10.1093/ccc/tcy011

Eyles, J., \& Smith, D. M. (1988). Qualitative methods in human geography. Polity.

Festinger, L. (1954). A theory of social comparison processes. Human Relations, 7(2), 117140. https://doi.org/10.1177/001872675400700202

Fitzsimons, J. (2011). Motivating people on climate change - is it a question of values? EnergyWatch, 60, 4-7. 
Glaser, B. G., \& Strauss, A. L. (1967). The discovery of grounded theory: Strategies for qualitative research. Aldine Publishing.

Gonick, L., \& Kasser, T. (2018). Hyper-Capitalism: The modern economy, its values, and how to change them. Brunswick, Victoria: Scribe Publications.

Grouzet, F. M. E., Kasser, T., Ahuvia, A., Dols, J. M. F., Kim, Y., Lau, S., Ryan, R. M., Saunders, S., Schmuck, P., \& Sheldon, K. M. (2005). The structure of goal contents across 15 cultures. Journal of Personality and Social Psychology, 89(5), 800-816. https://doi.org/10.1037/0022-3514.89.5.800

Hamilton, C. (2004). Growth fetish. Pluto Press.

Hamilton, C. (2005). Affluenza: When too much is never enough. Allen \& Unwin.

Harrison, J. (2018). The monetization of opinions: Consumer responses to covert endorsement practices on Instagram. Journal of Promotional Communications, 6(3), Article 3.

http://www.promotionalcommunications.org/index.php/pc/article/view/144

Ho, H., \& Ito, K. (2019). Consumption-oriented engagement in social network sites: Undesirable influence on personal well-being. European Journal of Marketing, 112017-0809. https://doi.org/10.1108/EJM-11-2017-0809

Ho, H., Shin, W., \& Lwin, M. O. (2017). Social networking site use and materialistic values among youth: The safeguarding role of the parent-child relationship and selfregulation. Communication Research, 0093650216683775. https://doi.org/10.1177/0093650216683775 Holowka, E. M. (2018). Between artifice and emotion: The "sad girls" of Instagram. Leadership, Popular Culture and Social Change. https://www.elgaronline.com/view/edcoll/9781785368967/9781785368967.00022 .$x m l$ 
Howell, R. A. (2013). It's not (just) "the environment, stupid!" Values, motivations, and routes to engagement of people adopting lower-carbon lifestyles. Global Environmental Change, 23(1), 281-290. https://doi.org/10.1016/j.gloenvcha.2012.10.015

Hurst, M., Dittmar, H., Bond, R., \& Kasser, T. (2013). The relationship between materialistic values and environmental attitudes and behaviors: A meta-analysis. Journal of Environmental Psychology, 36, 257.

Hwnag, H. S. (2019). Why social comparison on Instagram matters: Its impact on depression. KSII Transactions on Internet and Information Systems, 13(3), 16261638. https://doi.org/10.3837/tils.2019.03.029

IPCC. (2018). Summary for policymakers of IPCC special report on global warming of $1.5^{\circ} \mathrm{C}$ approved by governments. https://www.ipcc.ch/2018/10/08/summary-forpolicymakers-of-ipcc-special-report-on-global-warming-of-1-5c-approved-bygovernments/

Jackson, T. (2017). Prosperity without growth: Foundations for the economy of tomorrow (2nd edition.). Routledge.

Jungselius, B. (2019). "She liked the picture so i think she liked it". Unpacking the social practice of liking. Netcom, 33-1/2, 23-38. https://doi.org/10.4000/netcom.3849 Kasser, T. (2002a). Sketches for a self-determination theory of Values. In Handbook of selfdetermination theory research (p. 40). University Rochester Press.

Kasser, T. (2002b). The high price of materialism. MIT Press.

Kasser, T. (2016). Materialistic values and goals. Annual Review of Psychology, 67(1), 489514. https://doi.org/10.1146/annurev-psych-122414-033344

Kasser, T. (2019). An Overview of the Aspiration Index. Tim Kasser, Ph.D. https://www.timkasser.org 
Kasser, T., \& Ahuvia, A. (2002). Materialistic values and well-being in business students. European Journal of Social Psychology, 32(1), 137-146. https://doi.org/10.1002/ejsp.85

Kasser, T., \& Kanner, A. D. (2004). Psychology and consumer culture: The struggle for a good life in a materialistic world (1st ed.). American Psychological Association.

Kasser, T., \& Linn, S. (2016). Growing up under corporate capitalism: The problem of marketing to children, with suggestions for policy solutions. Social Issues and Policy Review, 10(1), 122-150. https://doi.org/10.1111/sipr.12020

Kasser, T., Rosenblum, K., Sameroff, A., Deci, E., Niemiec, C., Ryan, R., Árnadóttir, O., Bond, R., Dittmar, H., Dungan, N., \& Hawks, S. (2014). Changes in materialism, changes in psychological well-being: Evidence from three longitudinal studies and an intervention experiment. Motivation and Emotion, 38(1), 1-22.

https://doi.org/10.1007/s11031-013-9371-4

Kasser, T., \& Ryan, R. M. (1996). Further examining the american dream: Differential correlates of intrinsic and extrinsic goals. Personality and Social Psychology Bulletin, 22(3), 280-87. https://doi.org/10.1177/0146167296223006

Kasser, T., \& Ryan, R. M. (2001). Be careful what you wish for: Optimal functioning and the relative attainment of intrinsic and extrinsic goals. In Life goals and well-being: Towards a positive psychology of human striving (pp. 116-131). Hogrefe \& Huber Publishers.

Khamis, S., Ang, L., \& Welling, R. (2017). Self-branding, 'micro-celebrity' and the rise of Social Media Influencers. Celebrity Studies, 8(2), 191-208.

https://doi.org/10.1080/19392397.2016.1218292

Kim, H. (Christian). (2013). Situational materialism: How entering lotteries may undermine self-control. Journal of Consumer Research, 40(4), 759-772.

https://doi.org/10.1086/673191 
Krause, H.-V., Wagner, A., Krasnova, H., Deters, F. große, Baumann, A., \& Buxmann, P. (2019). Keeping up with the Joneses: Instagram use and its influence on conspicuous consumption. ICIS 2019 Proceedings. https://aisel.aisnet.org/icis2019/crowds_social/crowds_social/21

Ku, L., \& Zaroff, C. (2014). How far is your money from your mouth? The effects of intrinsic relative to extrinsic values on willingness to pay and protect the environment. Journal of Environmental Psychology, 40, 472-483. https://doi.org/10.1016/j.jenvp.2014.10.008

Kuipers, G. (2009). Humor styles and symbolic boundaries. Journal of Literary Theory, 3(2), 219-239. https://doi.org/10.1515/JLT.2009.013

Lambert, N. M., Fincham, F. D., Stillman, T. F., \& Dean, L. R. (2009). More gratitude, less materialism: The mediating role of life satisfaction. The Journal of Positive Psychology, 4(1), 32-42. https://doi.org/10.1080/17439760802216311

Lee, E., Lee, J.-A., Moon, J. H., \& Sung, Y. (2015). Pictures speak louder than words: Motivations for using Instagram. Cyberpsychology, Behavior, and Social Networking, 18(9), 552-556. https://doi.org/10.1089/cyber.2015.0157

Lekes, N., Hope, N. H., Gouveia, L., Koestner, R., \& Philippe, F. L. (2012). Influencing value priorities and increasing well-being: The effects of reflecting on intrinsic values. The Journal of Positive Psychology, 7(3), 249-261. https://doi.org/10.1080/17439760.2012.677468

Lodder, P., Ong, H. H., Grasman, R. P. P. P., \& Wicherts, J. M. (2019). A comprehensive meta-analysis of money priming. Journal of Experimental Psychology: General, 148(4), 688-712. https://doi.org/10.1037/xge0000570

Loukianov, A., Burningham, K., \& Jackson, T. (2020). Young people, good life narratives, and sustainable futures: The case of Instagram. Sustainable Earth, 3(1), 11. https://doi.org/10.1186/s42055-020-00033-2 
Lup, K., Trub, L., \& Rosenthal, L. (2015). Instagram \#Instasad?: Exploring associations among Instagram use, depressive symptoms, negative social comparison, and strangers followed. Cyberpsychology, Behavior, and Social Networking, 18(5), 247-252. https://doi.org/10.1089/cyber.2014.0560

Mandel, N., Petrova, P. K., \& Cialdini, R. B. (2006). Images of success and the preference for luxury brands. Journal of Consumer Psychology, 16(1), 57-69. https://doi.org/10.1207/s15327663jcp1601_8

Marcus, J., Ceylan, S., \& Ergin, C. (2017). Not so "traditional" anymore? Generational shifts on Schwartz values in Turkey. Journal of Cross-Cultural Psychology, 48(1), 58-74. https://doi.org/10.1177/0022022116673909

McCabe, J. L., \& Holmes, D. (2009). Reflexivity, critical qualitative research and emancipation: A Foucauldian perspective. Journal of Advanced Nursing, 65(7), 1518-1526. https://doi.org/10.1111/j.1365-2648.2009.04978.x

Meixner, B., \& Marlow, J. (2017). "Like" it or not: How do users understand the relationship between "likes" and edited social media content? 127655, 1893-1900. https://doi.org/10.1145/3027063.3053119

Michel, C., Oswald, A., Proto, E., \& Sovinsky, M. (2019). Advertising as a major source of human dissatisfaction: Cross-national evidence on one million Europeans. IDEAS Working Paper Series from RePEc.

http://search.proquest.com/docview/2186319998/?pq-origsite=primo

Miltner, K. M. (2014). "There's no place for lulz on LOLCats": The role of genre, gender, and group identity in the interpretation and enjoyment of an Internet meme. First Monday. https://doi.org/10.5210/fm.v19i8.5391

Moldes, O., \& Ku, L. (2020). Materialistic cues make us miserable: A meta-analysis of the experimental evidence for the effects of materialism on individual and societal well-being. Psychology \& Marketing, n/a(n/a). https://doi.org/10.1002/mar.21387 
Molinsky, A. L., Grant, A. M., \& Margolis, J. D. (2012). The bedside manner of homo economicus: How and why priming an economic schema reduces compassion. Organizational Behavior and Human Decision Processes, 119(1), 27-37. https://doi.org/10.1016/j.obhdp.2012.05.001

Monbiot, G. (2014). The values ratchet. George Monbiot. https://www.monbiot.com/2014/06/10/the-values-ratchet/

Murray, D. C. (2015). Notes to self: The visual culture of selfies in the age of social media. Consumption Markets \& Culture, 18(6), 490-516. https://doi.org/10.1080/10253866.2015.1052967

Newlands, G., \& Fieseler, C. (2020). \#dreamjob: Navigating pathways to success as an aspiring Instagram influencer. The Regulation of Social Media Influencers. https://www.elgaronline.com/view/edcoll/9781788978279/9781788978279.00016 .$x \mathrm{ml}$

O'Leary, Z. (2014). The essential guide to doing your research project (2nd edition.). SAGE. Olszanowski, M. (2014). Feminist self-imaging and Instagram: Tactics of circumventing sensorship. Visual Communication Quarterly: Legal, Ethical, and Technical Challenges in the Online \& Mobile Visual Paradigm, 21(2), 83-95. https://doi.org/10.1080/15551393.2014.928154

Page, R. (2012). The linguistics of self-branding and micro-celebrity in Twitter: The role of hashtags. Discourse \& Communication, 6(2), 181-201. https://doi.org/10.1177/1750481312437441

Pardes, A. (2017). Selfie factories: The rise of the made-for-Instagram museum. Wired. https://www.wired.com/story/selfie-factories-instagram-museum/

Phan, K., \& Macias, M. (2018). How Instagram is changing the art world. Southern California Conferences for Undergraduate Research. https://www.sccur.org/sccur/FALL_2018_CONFERENCE/SOC_SCI_POSTERS/82 
Piurko, Y., Schwartz, S. H., \& Davidov, E. (2011). Basic personal values and the meaning of left-right political orientations in 20 countries. Political Psychology, 32(4), 537-561. https://doi.org/10.1111/j.1467-9221.2011.00828.x

Rainie, L., \& Zickuhr, K. (2015). Americans' views on mobile etiquette. Pew Research Centre. https://www.pewresearch.org/internet/2015/08/26/americans-views-on-mobileetiquette/

Raworth, K. (2017). Doughnut economics: Seven ways to think like a 21st century economist. Chelsea Green Publishing.

Richins, M. L. (2017). Materialism pathways: The processes that create and perpetuate materialism. Journal of Consumer Psychology, 27(4), 480-499. https://doi.org/10.1016/j.jcps.2017.07.006

Richins, M. L., \& Dawson, S. (1992). A consumer values orientation for materialism and its measurement: Scale development and validation. Journal of Consumer Research, 19(3), 303-316. https://doi.org/10.1086/209304

Ripple, W. J., Wolf, C., Newsome, T. M., Barnard, P., \& Moomaw, W. R. (2020). World scientists' warning of a climate emergency. BioScience, 70(1), 8-12. https://doi.org/10.1093/biosci/biz088

Roberts, J. A., \& David, M. E. (2016). My life has become a major distraction from my cell phone: Partner phubbing and relationship satisfaction among romantic partners. Computers in Human Behavior, 54, 134-141. https://doi.org/10.1016/j.chb.2015.07.058

Rockström, J., Steffen, W., Noone, K., Persson, Å., Chapin, F. S., Lambin, E., Lenton, T. M., Scheffer, M., Folke, C., Schellnhuber, H. J., Nykvist, B., de Wit, C. A., Hughes, T., van der Leeuw, S., Rodhe, H., Sörlin, S., Snyder, P. K., Costanza, R., Svedin, U., ... Foley, J. (2009). Planetary boundaries: Exploring the safe operating space for humanity. Ecology and Society, 14(2). JSTOR. https://www.jstor.org/stable/26268316 
Rooney, D. (2000). Thirty years of competition in the British tabloid press: The Mirror and the Sun 1968-1998. In Tabloid Tales: Global Debates Over Media Standards. Rowman \& Littlefield.

RSPH. (2017). \#StatusOfMind: Social media and young people's mental health and wellbeing. Royal Society for Public Health. https://www.rsph.org.uk/ourwork/campaigns/status-of-mind.html

Ryan, R. M., \& Deci, E. L. (2017). Self-determination theory: Basic psychological needs in motivation, development, and wellness. Guilford Press.

Sapolsky, R. M. (2017). Behave: The biology of humans at our best and worst. Penguin Press.

Schultz, P. W., Gouveia, V. V., Cameron, L. D., Tankha, G., Schmuck, P., \& Franěk, M. (2005). Values and their relationship to environmental concern and conservation behavior. Journal of Cross-Cultural Psychology, 36(4), 457-475. https://doi.org/10.1177/0022022105275962

Schwartz, S. H. (1992). Universals in the content and structure of values: Theoretical advances and empirical tests in 20 countries. In M. P. Zanna (Ed.), Advances in experimental social psychology, Vol. 25 (pp. 1-65, Chapter ix, 390 Pages). Academic Press (San Diego, CA, US). http://dx.doi.org/10.1016/S0065-2601(08)60281-6

Schwartz, S. H. (2011). Studying values: Personal adventure, future directions. 307-319.

Schwartz, S. H., Cieciuch, J., Vecchione, M., Davidov, E., Fischer, R., Beierlein, C., Ramos, A., Verkasalo, M., Lönnqvist, J.-E., Demirutku, K., Dirilen-Gumus, O., \& Konty, M. (2012). Refining the theory of basic individual values. Journal of Personality and Social Psychology, 103(4), 663-688. https://doi.org/10.1037/a0029393

Senker, P. (2011). Prosperity without growth: Economics for a finite planet. Energy \& Environment, 22(7). https://tewaharoa.victoria.ac.nz 
Sheldon, P., \& Bryant, K. (2016). Instagram: Motives for its use and relationship to narcissism and contextual age. Computers in Human Behavior, 58, 89-97. https://doi.org/10.1016/j.chb.2015.12.059

Sherlock, M., \& Wagstaff, D. L. (2019). Exploring the relationship between frequency of Instagram use, exposure to idealized images, and psychological well-being in women. Psychology of Popular Media Culture, 8(4), 482-490.

https://doi.org/10.1037/ppm0000182

Shrubsole, G. (2012). Consumers outstrip Citizens in the British media. OpenDemocracy. https://www.opendemocracy.net/en/opendemocracyuk/consumers-outstripcitizens-in-british-media/

Silverman, D. (2011). Interpreting qualitative data: A guide to the principles of qualitative research (4th ed.). Sage.

Smith, S. P. (2018). Instagram abroad: Performance, consumption and colonial narrative in tourism. Postcolonial Studies, 21(2), 172-191. https://doi.org/10.1080/13688790.2018.1461173

Srinivasan, R. (2017). After the Internet. Polity Press.

Stanley, L. (1983). Breaking out: Feminist consciousness and feminist research. Routledge \& KPaul.

Statista. (2020). Instagram. Statista. https://www.statista.com/study/21392/instagramstatista-dossier/

Stewart, R. (2018). UK shoppers say brands aren't transparent about influencer deals and rules need tightened. The Drum. https://www.thedrum.com/news/2018/02/12/ukshoppers-say-brands-arent-transparent-about-influencer-deals-and-rules-need

Streck, C., Unger, M. V., \& Greiner, S. (2020). COP 25: Losing sight of (raising) ambition. Journal for European Environmental \& Planning Law, 17(2), 136-160. https://doi.org/10.1163/18760104-01702003 
Stuart, D., Gunderson, R., \& Petersen, B. (2020a). Overconsumption as ideology: Implications for addressing global climate change. Nature and Culture, 15(2). https://doi.org/10.3167/nc.2020.150205

Stuart, D., Gunderson, R., \& Petersen, B. (2020b). The climate crisis as a catalyst for emancipatory transformation: An examination of the possible. International Sociology, 35(4), 433-456. https://doi.org/10.1177/0268580920915067

Suler, J. (2005). The online disinhibition effect. International Journal of Applied Psychoanalytic Studies, 2(2), 184-188. https://doi.org/10.1002/aps.42

Sundie, J. M., Kenrick, D. T., Griskevicius, V., Tybur, J. M., Vohs, K. D., \& Beal, D. J. (2011). Peacocks, porsches, and Thorstein Veblen: Conspicuous consumption as a sexual signaling system. Journal of Personality and Social Psychology, 100(4), 664-680. http://dx.doi.org/10.1037/a0021669

Tashakkori, A. (1998). Mixed methodology: Combining qualitative and quantitative approaches. Sage.

Tolentino, J. (2020). Trick mirror: Reflections on self delusion. Random House Trade Paperbacks.

Twenge, J. M. (2017). IGen: Why today's super-connected kids are growing up less rebellious, more tolerant, less happy-- and completely unprepared for adulthood (and what this means for the rest of us) (First Atria books hardcover edition.). Atria Books.

Twenge, J. M., Campbell, W. K., \& Freeman, E. C. (2012). Generational differences in young adults' life goals, concern for others, and civic orientation, 1966-2009. Journal of Personality and Social Psychology, 102(5), 1045-1062.

https://doi.org/10.1037/a0027408

Unanue, W., Vignoles, V. L., Dittmar, H., \& Vansteenkiste, M. (2016). Life goals predict environmental behavior: Cross-cultural and longitudinal evidence. Journal of 
Environmental Psychology, 46(C), 10-22.

https://doi.org/10.1016/j.jenvp.2016.02.001

Vandenkerckhove, B., Brenning, K., Vansteenkiste, M., Luyten, P., \& Soenens, B. (2019). The explanatory role of basic psychological need experiences in the relation between dependency, self-criticism and psychopathology in adolescence. Journal of Psychopathology and Behavioral Assessment, 41(4), 574-588.

https://doi.org/10.1007/s10862-019-09719-0

Venhoeven, L., Bolderdijk, J. W., \& Steg, L. (2013). Explaining the paradox: How proenvironmental behaviour can both thwart and foster well-being. Sustainability, 5(4), 1372-1386. https://doi.org/10.3390/su5041372

Vogel, E. A., Rose, J. P., Okdie, B. M., Eckles, K., \& Franz, B. (2015). Who compares and despairs? The effect of social comparison orientation on social media use and its outcomes. Personality and Individual Differences, 86, 249-256. https://doi.org/10.1016/j.paid.2015.06.026

Vohs, K. D., Mead, N. L., \& Goode, M. R. (2006). Psychological Consequences of Money. Science, 314(5802), 1154-1156. https://doi.org/10.1126/science.1132491

Wang, X., \& Li, Y. (2014). Trust, psychological need, and motivation to produce usergenerated content: A self-determination perspective. Journal of Electronic Commerce Research, 15(3), 241-253.

Weinstein, N., Przybylski, A. K., \& Ryan, R. M. (2009). Can nature make us more caring? Effects of immersion in nature on intrinsic aspirations and generosity. Personality and Social Psychology Bulletin, 35(10), 1315-1329.

https://doi.org/10.1177/0146167209341649

Winter, S., Maslowska, E. H., \& Vos, A. L. (2020). The effects of trait-based personalization in social media advertising. Computers in Human Behavior, 106525. https://doi.org/10.1016/j.chb.2020.106525 
Wu, T. (2019). Blind spot: The attention economy and the law. Antitrust Law Journal, 82(3), 771-806.

Zelenski, J. M., Dopko, R. L., \& Capaldi, C. A. (2015). Cooperation is in our nature: Nature exposure may promote cooperative and environmentally sustainable behavior. Journal of Environmental Psychology, 42, 24-31.

https://doi.org/10.1016/j.jenvp.2015.01.005 


\title{
Appendices
}

\author{
Appendix A: Ethics approval
}

A Human Ethics application has been approved. 0000027774 Automated Email, Do Not Repl)

R researchmaster-help@vuw.ac.nz

$\leftrightarrow$ Reply $\quad$ « Reply All $\rightarrow$ Forward $\quad \cdots$

Cc lollystray@gmail.com

Fri 6/09/2019 4:14 P|

Dear Head of School or delegate,

A Human Ethics application has been approved for a researcher in your School. The application details are below. You have online access to this form through ResearchMaster here: https://rme6.vuw.ac.nz/RME6/.

Application ID: 0000027774

Application title: The effect of social media on activating extrinsic values

Researcher: Melanie Vautier

It is best to use either Internet Explorer or Safari to access the system.

Thank you

ResearchMaster

****** This is an automated email. Do not reply to this email address $* * * * * * *$

Queries for the central Human Ethics Committee can be sent to ethicsadmin@vuw.ac.nz

Pipitea Ethics subcommittee queries can be sent to: pipitea-hec@vuw.ac.nz

Psychology Ethics subcommittee queries can be sent to: louise.hamblin@vuw.ac.nz

Human Ethics amendment/extension request approved 0000027774 Automated Email

R researchmaster-help@vuw.ac.nz

To Iollystray@gmail.com; Isobel Cairns; HEC; Melanie Vautier

Cc Wokje Abrahamse

Dear Melanie,

Your application for amendment/extension of Human Ethics application number 0000027774 (The effect of social media on activating extrinsic values) is approved as of today.

In the case of an amendment, this approval is valid until the end date of your original ethics approval; in the case of an extension, this approval applies until the new end date that you have nominated.

If you would like to receive a formal letter please contact the HEC Administrator (ethicsadmin@vuw.ac.nz).

Best wishes with the research,

Judith Loveridge

HEC Convenor

*****This is an automated email. Do not reply to this email address $* * * * * * *$

Queries for the central Human Ethics Committee can be sent to ethicsadmin@vuw.ac.nz

Pipitea Ethics subcommittee queries can be sent to: pipitea-hec@vuw.ac.nz

Psychology Ethics subcommittee queries can be sent to: louise.hamblin@vuw.ac.nz 


\section{Effects of social media on activating extrinsic values}

\section{INFORMATION SHEET FOR PARTICIPANTS}

You are invited to take part in this research. Please read this information before deciding whether or not to take part. If you decide to participate, thank you. If you decide not to participate, thank you for considering this request.

\section{Who am I?}

My name is Melanie Vautier and I am a Masters student in Environmental Studies at Victoria University of Wellington. This research project is work towards my thesis.

\section{What is the aim of the project?}

This project aims to explore experiences of Instagram, including identifying ways that social media may foster materialistic values; which are closely correlated with lower wellbeing, less environmental concern, and a higher carbon footprint.

Your participation will support this research by sharing your experience and perspective on these issues, and how the content you see on your own social media relates to different value systems.

This research has been approved by the Victoria University of Wellington Human Ethics Committee, reference number $\# 0000027774$

\section{How can you help?}

You have been invited to participate because you will offer a valuable perspective of your own life experience, how you use social media and how you perceive it affects you. If you agree to take part I will interview you at a mutually agreed location, such as a central Wellington café. I will ask you questions about your views and 
experiences of social media. The interview will take one hour. I will audio record the interview with your permission and write it up later. You can choose to not answer any question or stop the interview at any time, without giving a reason. You can withdraw from the study by contacting me at any time before December $30^{\text {th }}$, 2019. If you withdraw, the information you provided will be destroyed or returned to you.

\section{What will happen to the information you give?}

This research is confidential. This means that the researchers named below will be aware of your identity, but the research data will be combined and your identity will not be revealed in any reports, presentations, or public documentation.

Only my supervisor and I will read the notes or transcript of the interview. The interview transcripts, summaries and any recordings will be kept securely and destroyed on the $1^{\text {st }}$ October 2021.

\section{What will the project produce?}

The information from my research will be used in my Masters thesis. There is also potential that it will be used in academic publications and presentations.

If you accept this invitation, what are your rights as a research participant?

You do not have to accept this invitation if you don't want to. If you do decide to participate, you have the right to:

- $\quad$ choose not to answer any question;

- $\quad$ ask for the recorder to be turned off at any time during the interview

- $\quad$ withdraw from the study before November 5 $5^{\text {th }}, 2019$

- $\quad$ ask any questions about the study at any time;

- $\quad$ receive a copy of your interview recording; 
- be able to read any reports of this research by emailing the researcher to request a copy.

If you have any questions or problems, who can you contact?

If you have any questions, either now or in the future, please feel free to contact either:

Student:

Name: Melanie Vautier

Email address:

melanie.vautier@vuw.ac.nz
Supervisor:

Name: Wokje Abrahamse

Role: Lecturer

School: School of Geography, Environment and Earth Sciences

wokje.abrahamse@vuw.ac.nz

Human Ethics Committee information

If you have any concerns about the ethical conduct of the research you may contact the Victoria University HEC Convenor: Dr Judith Loveridge. Email hec@vuw.ac.nz or telephone $+64-4-4636028$. 


\section{Effects of social media on activating extrinsic values}

\section{CONSENT TO INTERVIEW}

This consent form will be held for five years.

Researcher: Melanie Vautier, Victoria University of Wellington.

- I have read the Information Sheet and the project has been explained to me. My questions have been answered to my satisfaction. I understand that I can ask further questions at any time.

- I agree to take part in an audio recorded interview.

I understand that:

- I may withdraw from this study at any point before December $30^{\text {th }} 2019$, and any information that I have provided will be returned to me or destroyed.

- $\quad$ The identifiable information I have provided will be destroyed on $1^{\text {st }}$ October 2021.

- $\quad$ Any information I provide will be kept confidential to the researcher and the supervisor.

- I understand that the findings may be used for a Masters thesis, with the potential to be used in academic publications and presentations.

- I understand that the recordings will be kept confidential to the researcher and the supervisor.

- My name will not be used in reports and utmost care will be taken not to disclose any information that would identify me.

- I would like a copy of the recording of my interview:

Yes $\square \quad$ No

- I would like to receive a copy of the final report and have added my email address below.

Yes $\square \quad$ No

Signature of participant:

Name of participant:

Date: 
Contact details:

Appendix C: Group interview information and consent form

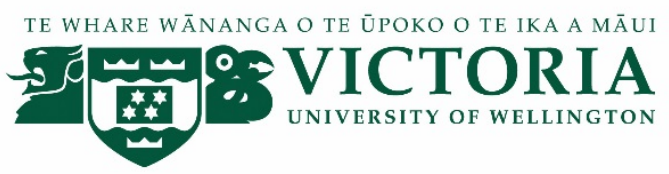

\section{Is social media showing you what you want to see?}

\section{INFORMATION SHEET FOR PARTICIPANTS FOR FOCUS GROUP}

You are invited to take part in this research. Please read this information before deciding whether or not to take part. If you decide to participate, thank you. If you decide not to participate, thank you for considering this request.

\section{Who am I?}

My name is Melanie Vautier and I am a Masters student in Environmental Studies at Victoria University of Wellington. This research project is work towards my thesis.

\section{What is the aim of the project?}

This project aims to explore experiences of Instagram, including identifying ways that social media may foster stronger materialistic values; which are closely correlated with lower wellbeing, less environmental concern, and a higher carbon footprint.

Your participation will support this research by sharing your experience and perspective on these issues, and how the content you see on your own social media relates to different value systems.

This research has been approved by the Victoria University of Wellington Human Ethics Committee, reference number \#0000027774

\section{How can you help?}

You have been invited to participate because you are an active user of social media. If you agree to take part, you will be part of a focus group at Victoria University of Wellington. I will ask you and other participants questions about the content you are exposed to on social media and how you perceive it affects you. The focus group will take a maximum of two hours. I will audio record the focus group with your permission and write it up later. 
The information shared during the focus group is confidential. That means after the focus group, you may not communicate to anyone, including family members and close friends, any details about the identities or contributions of the other participants of the focus group.

You can withdraw from the focus group at any time before the focus group begins. You can also withdraw while the focus group it is in progress. However it will not be possible to withdraw the information you have provided up to that point as it will be part of a discussion with other participants.

\section{What will happen to the information you give?}

This research is confidential. This means that the researcher named below will be aware of your identity but the research data will be combined and your identity will not be revealed in any reports, presentations, or public documentation.

Only my supervisor and I will read the notes or transcript of the focus group. The focus group transcripts, summaries and any recordings will be kept securely and destroyed on the $1^{\text {st }}$ of October 2021.

\section{What will the project produce?}

The information from my research will be used in my Masters thesis. There is also potential that it will be used in academic publications and presentations.

\section{If you accept this invitation, what are your rights as a research participant?}

You do not have to accept this invitation if you don't want to. If you do decide to participate, you have the right to:

- $\quad$ choose not to answer any question;

- $\quad$ ask for the recorder to be turned off at any time during the focus group;

- withdraw from the focus group while it is taking part however it will not be possible to withdraw the information you have provided up to that point;

- $\quad$ ask any questions about the study at any time;

- be able to read any reports of this research by emailing the researcher to request a copy.

\section{If you have any questions or problems, who can you contact?}

If you have any questions, either now or in the future, please feel free to contact [either/me]:

Student:

Supervisor: 
Name: Melanie Vautier

Email address:

melanie.vautier@vuw.ac.nz
Name: Wokje Abrahamse

Role: Lecturer

School: School of Geography, Environment and Earth Sciences

wokje.abrahamse@vuw.ac.nz

\section{Human Ethics Committee information}

If you have any concerns about the ethical conduct of the research you may contact the Victoria University HEC Convenor: Dr Judith Loveridge. Email hec@vuw.ac.nz or telephone +64-4-463 6028 .

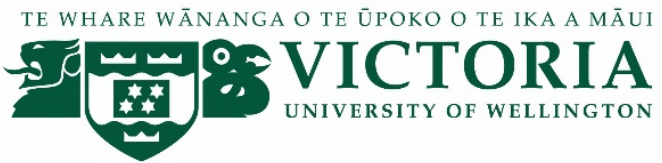

\section{Effects of social media on activating extrinsic values}

\section{CONSENT TO PARTICIPATE IN FOCUS GROUP}

This consent form will be held for five years.

Researcher: Melanie Vautier, School of Geography, Environment and Earth Sciences, Victoria University of Wellington.

- I have read the Information Sheet and the project has been explained to me. My questions have been answered to my satisfaction. I understand that I can ask further questions at any time.

- I agree to take part in an audio recorded focus group.

I understand that:

- I acknowledge that I am agreeing to keep the information shared during the focus group confidential. I am aware that after the focus group, I must not communicate to anyone, including family members and close friends, any 
details about the identities or contributions of the other participants of the focus group.

- I can withdraw from the focus group while it is in progress however it will not be possible to withdraw the information I have provided up to that point as it will be part of a discussion with other participants

- The identifiable information I have provided will be destroyed on October $1^{\text {st }}$, 2021.

- I understand that the findings may be used for a Masters thesis, with the potential to be used in academic publications and presentations.

- I understand that the recordings will be kept confidential to the researcher and the supervisor.

- $\quad$ My name will not be used in reports and utmost care will be taken not to disclose any information that would identify me.

Signature of participant:

Name of participant:

Date:

Contact details: 


\begin{tabular}{|l|l|}
\hline General theme & Questions \\
\hline Initial questions & When did you get Instagram? Why? \\
\hline How long do you spend on it?
\end{tabular}




\begin{tabular}{|l|l|}
\hline Values and aspirations & $\begin{array}{l}\text { What values do you see most portrayed on your } \\
\text { Instagram? }\end{array}$ \\
& Would you like to be an influencer? \\
\hline Any other comments & Misc / open \\
\hline
\end{tabular}

